\title{
The Dolgopyat inequality in bounded variation for non-Markov maps
}

\author{
Henk Bruin and Dalia Terhesiu
}

December 2016

\begin{abstract}
Let $F$ be a (non-Markov) countably piecewise expanding interval map satisfying certain regularity conditions, and $\tilde{\mathcal{L}}$ the corresponding transfer operator. We prove the Dolgopyat inequality for the twisted operator $\tilde{\mathcal{L}}_{s}(v)=\tilde{\mathcal{L}}_{s}\left(e^{s \varphi} v\right)$ acting on the space BVof functions of bounded variation, where $\varphi$ is a piecewise $C^{1}$ roof function.
\end{abstract}

\section{Introduction}

A crucial method (including what is now known as the Dolgopyat inequality) to prove exponential decay of correlations for Anosov flows with $C^{1}$ stable and unstable foliations was developed by Dolgopyat [7]. Liverani [10] obtained exponential decay of correlations for Anosov flows with contact structure (and hence geodesic flow on compact negatively curved manifolds of any dimension).

Baladi \& Vallée [4] further refined the method of [7] to prove exponential decay of correlations for suspension semiflows over one-dimensional piecewise $C^{2}$ expanding Markov maps with $C^{1}$ roof functions. This was extended to the multidimensional setting by Avila et al. [3], to prove exponential decay of correlations of Teichmüller flows. Araújo \& Melbourne [1] showed that the method can be adapted to suspension semiflows over $C^{1+\alpha}$ maps with $C^{1}$ roof functions, which enabled them to prove that the classical Lorenz attractor has exponential decay of correlations.

In all of the above works, the results are applied to $C^{\alpha}$ observables for some $\alpha>0$. In this paper, we consider a class of non-Markov maps (see Section 2), obtain a Dolgopyat inequality on the space of bounded variation (BV) observables (Theorem 2.3). The Dolgopyat inequality obtained in this paper automatically allows us to obtain exponential decay of correlations for skew-products on $\mathbb{T}^{2}$ as considered by Butterley and Eslami [6, 8], where the developed methods do not exploit the presence of the Markov structure.

Most probably, a proof of exponential decay for BV observables for the class of non Markov maps considered here is not the easiest route; one could, for instance, think of inducing to a Markov map for which exponential decay of correlation of $C^{2}$ observables is known and then use approximation arguments to pass to BV observables. Instead, we believe that the benefit of the Dolgopyat inequality in this setting is that it can be used to study perturbations of the flow (such as inserting holes in the Poincaré map); it is not at all clear that this can be economically done via inducing.

The main new ingredient of the proof is to locate and control the sizes of the jumps associated with BV functions (see Section 4).

Faculty of Mathematics, University of Vienna, Oskar Morgensternplatz 1, 1090 Vienna, Austria; e-mail: henk.bruin@univie.ac.at

College of Engineering, Mathematics and Physical Sciences Harrison Building Streatham Campus, University of Exeter, North Park Road, Exeter EX4 4QF, UK; e-mail: daliaterhesiu@ gmail.com 


\subsection{Specific Examples}

Our results (i.e., the Dolgopyat type inequality given by Theorem 2.3) apply to typical AFU maps presented in Section 2. By typical we mean the whole clas of AFU maps (studied by Zweimüller $[13,14])$ satisfying assumption (2.5) below. This assumption is very mild, see Remark 2.2. In particular, this class contains some standard families, such as the shifted $\beta$-transformations $F$ : $[0,1] \rightarrow[0,1], x \mapsto \beta x+\alpha(\bmod 1)$ for fixed $\alpha \in[0,1)$ and $\beta>1$.

Another important example is the First Return Map of a (non-Markov) Manneville-Pomeau map. That is,

$$
F=f^{\tau}:\left[\frac{1}{2}, 1\right] \rightarrow\left[\frac{1}{2}, 1\right] \quad \text { for } \quad \tau(x)=\min \left\{n \geq 1: f^{n}(x) \in\left[\frac{1}{2}, 1\right]\right\},
$$

where

$$
f:[0,1] \rightarrow[0,1], \quad x \mapsto \begin{cases}x\left(1+2^{\alpha} x^{\alpha}\right) & x \in\left[0, \frac{1}{2}\right) ; \\ \gamma(2 x-1) & x \in\left[\frac{1}{2}, 1\right],\end{cases}
$$

is a non-Markov Manneville-Pomeau map with fixed $\alpha>0$ and $\gamma \in\left(\frac{1}{2}, 1\right]$.

The assumptions below apply to these to these examples, albeit that (2.5) holds for all parameters with the exception of a set of Hausdorff dimension $<1$, see Remark 2.2. The UNI condition (2.9) is a generic condition on the roof function of the type previously considered in $[4,3]$.

\section{Set-up, notation, assumptions and results.}

We start this section by discussing the class of AFU maps studied by Zweimüller [13, 14]. We present their conditions in Subsections 2.1-2.6.

\subsection{The AFU map $F$.}

Let $Y$ be an interval and $F: Y \rightarrow Y$ a topologically mixing piecewise $C^{2}$ AFU map (i.e., uniformly expanding with finite image partition and satisfying Adler's condition), preserving a probability measure $\mu$ which is absolutely continuous w.r.t. Lebesgue measure Leb. Let $\alpha$ be the partition of $Y$ into domains of the branches of $F$, and $\alpha^{n}=\bigvee_{i=0}^{n-1} F^{-i} \alpha$. Thus $F^{n}: a \rightarrow F^{n}(a)$ is a monotone diffeomorphism for each $a \in \alpha^{n}$. The collection of inverse branches of $F^{n}$ is denoted as $\mathcal{H}_{n}$, and each $h \in \mathcal{H}_{n}$ is associated to a unique $a \in \alpha^{n}$ such that $h: F^{n}(a) \rightarrow a$ is a contracting diffeomorphism.

\subsection{Uniform expansion.}

Let

$$
\rho_{0}=\inf _{x \in Y}\left|F^{\prime}(x)\right| \quad \text { and } \quad \rho=\rho_{0}^{1 / 4} .
$$

Since $F$ is uniformly expanding, $\rho_{0}>\rho>1$, but in fact, we will assume that $\rho_{0}>2^{4 / 3}$, which can be achieved by taking an iterate.

\subsection{Adler's condition.}

This condition states that $\sup _{a \in \alpha} \sup _{x \in a} \frac{\left|F^{\prime \prime}(x)\right|}{\left|F^{\prime}(x)\right|^{2}}<\infty$. As $F$ is expanding, $\frac{\left|\left(F^{n}\right)^{\prime \prime}(x)\right|}{\left|\left(F^{n}\right)^{\prime}(x)\right|^{2}}$ is bounded uniformly over the iterates $n \geq 1, a \in \alpha^{n}$ and $x \in a$ as well. Thus, there is $C_{1} \geq 0$ such that

$$
\frac{\left|\left(F^{n}\right)^{\prime \prime}(h(x))\right|}{\left|\left(F^{n}\right)^{\prime}(h(x))\right|^{2}} \leq C_{1} \quad \text { and } \quad \frac{h^{\prime}(x)}{h^{\prime}\left(x^{\prime}\right)} \leq e^{C_{1}\left|x-x^{\prime}\right|}
$$

for all $n \geq 1, h \in \mathcal{H}_{n}$ and $x, x^{\prime} \in \operatorname{dom}(h)$. The second inequality follows from the first by a standard computation. 


\subsection{Finite image partition.}

The map $F$ need not preserve a Markov partition, but has the finite image property. Therefore $K:=\min \{|F(a)|: a \in \alpha\}$ is positive. We assume that $F$ is topologically mixing. This implies that there is $k_{1} \in \mathbb{N}$ such that $F^{k_{1}}(J) \subset Y$ for all intervals $J$ of length $|J| \geq \delta_{0}:=\frac{K\left(\rho_{0}-2\right)}{5 e^{C_{1} \rho_{0}}}$ (this choice of $\delta_{0}$ is used in Lemma B.1).

Let $X_{1}=X_{1}^{\prime}$ be the collection of boundary points of $F(a), a \in \alpha$, where $\alpha$ is the partition of $Y$ into branches of $F$. Due to the finite image property, $X_{1}$ is a finite collection of points; we denote its cardinality by $N_{1}$. Inductively, let $X_{k}^{\prime}=F\left(X_{k-1}^{\prime}\right)$, i.e., the set of "new" boundary points of the $k$-th image partition, and $X_{k}=\cup_{j \leq k} X_{j}^{\prime}$. Therefore $\# X_{k}^{\prime} \leq k N_{1}$. Let $\left\{\xi_{i}\right\}_{i=0}^{M}$ be a collection of points containing $X_{k}$, and put in increasing order, Then

$$
\mathcal{P}_{k}=\left\{\left(\xi_{i-1}, \xi_{i}\right): i=1, \ldots, M\right\}
$$

is a partition of $Y$, refining the image partition of $F^{k}$. In other words, the components of $Y \backslash$ $\left\{\xi_{i}\right\}_{i=0}^{M}$ are the atoms of $\mathcal{P}_{k}$.

\subsection{Roof function.}

Let $\varphi: Y \rightarrow \mathbb{R}^{+}$be a piecewise $C^{1}$ function, such that $\varphi \geq 1$ and

$$
C_{2}:=\sup _{h \in \mathcal{H}} \sup _{x \in \operatorname{dom}(h)}\left|(\varphi \circ h)^{\prime}(x)\right|<\infty .
$$

Since a main application is the decay of correlations of the vertical suspension semi-flow on $\{(y, u): y \in Y, 0 \leq u \leq \varphi(y)\} /(y, \varphi(y)) \sim(F(y), 0)$, see Subsection 2.9, we will call $\varphi$ the roof function.

Also assume that there is $\varepsilon_{0}>0$ such that

$$
C_{3}:=\sup _{x \in Y} \sum_{h \in \mathcal{H}, x \in \operatorname{dom}(h)}\left|h^{\prime}(x)\right| e^{\varepsilon_{0} \varphi \circ h(x)}<\infty .
$$

\subsection{Further assumption on $F$ (relevant for the non-Markov case)}

We first discuss some known properties of the transfer operator and twisted transfer operator. Let Leb denote Lebesque measure. Define the BV-norm $\|v\|_{\mathrm{BV}}$ of $v: I \rightarrow \mathbb{C}$, for an interval $I \subset \mathbb{R}$, as the sum of its $L^{1}$-norm (w.r.t. Leb) $\|v\|_{1}$ and the total variation $\operatorname{Var}_{I} v=$ $\inf _{\tilde{v}=v \text { a.e. }} \sup _{x_{0}<\cdots<x_{N} \in I} \sum_{i=1}^{N}\left|\tilde{v}\left(x_{i}\right)-\tilde{v}\left(x_{i-1}\right)\right|$.

Let $\mathcal{L}: L^{1}(Y$, Leb $) \rightarrow L^{1}(Y$, Leb $)$ be the transfer operators associated to $(Y, F)$ given by $\mathcal{L}^{n} v=\sum_{h \in \mathcal{H}_{n}}\left|h^{\prime}\right| v \circ h, n \geq 1$. For $s=\sigma+i b \in \mathbb{C}$, let $\mathcal{L}_{s}$ be the twisted version of $\mathcal{L}$ defined via $\mathcal{L}_{s} v=\mathcal{L}\left(e^{s \varphi} v\right)$ with iterates

$$
\mathcal{L}_{s}^{n} v=\sum_{h \in \mathcal{H}_{n}} e^{s \varphi_{n} \circ h}\left|h^{\prime}\right| v \circ h, \quad n \geq 1 .
$$

We first note that for $s=\sigma \in \mathbb{R}$,

Proposition 2.1. There exist $\varepsilon \in(0,1)$ such that for all $|\sigma|<\varepsilon$, $\left\|\mathcal{L}_{\sigma}\right\|_{B V}<\infty$.

Proof. By Remark A.1, there exist $c_{1}, c_{2}>0$ and $\varepsilon \in(0,1)$ such that $\operatorname{Var}_{Y}\left(\mathcal{L}_{\sigma} v\right) \leq c_{1} \operatorname{Var}_{Y} v+$ $c_{2}\|v\|_{\infty}$, for all $|\sigma|<\varepsilon$. Note that for any $v \in \mathrm{BV}(Y),\|v\|_{\infty} \leq \operatorname{Var}_{Y} v+\|v\|_{1}$. Hence, $\operatorname{Var}_{Y}\left(\mathcal{L}_{\sigma} v\right) \leq\left(c_{1}+c_{2}\right) \operatorname{Var}_{Y} v+c_{2}\|v\|_{1}$. Also, $\int_{Y}\left|\mathcal{L}_{\sigma} v\right| d$ Leb $\leq C_{2}\|v\|_{\infty} \leq C_{2}\left(\operatorname{Var}_{Y} v+\|v\|_{1}\right)$ and the conclusion follows.

It is known that $\mathcal{L}_{0}=\mathcal{L}$ has a simple eigenvalue $\lambda_{0}=1$ with eigenfunction $f_{0} \in \mathrm{BV}$, [13, Lemma 4] (see also [12]), and $\frac{1}{C_{4}} \leq f_{0}(x) \leq C_{4}$ for all $x \in Y$, see [14, Lemma 7]. Hence, $f_{0}$ is bounded from above and below. This together with Proposition 2.1 implies that there exists 
$\varepsilon \in(0,1)$ such that $\mathcal{L}_{\sigma}$ has a family of simple eigenvalues $\lambda_{\sigma}$ for $|\sigma|<\varepsilon$ with BV eigenfunctions $f_{\sigma}$.

We assumed above that $F$ has the finite image property, but not that $F^{n}$ has the finite image property uniformly over $n \geq 1$. We put a condition on $F$ as follows: the lengths of the atoms $p \in \mathcal{P}_{k}$, with $k$ specified below, do not decrease faster than $\rho^{-k}$ :

$$
\min _{p \in \mathcal{P}_{k}} \operatorname{Leb}(p)>\frac{16 C_{8}}{C_{9}} \frac{\sup f_{\sigma}}{\inf f_{\sigma}} \rho^{-k}
$$

where $C_{8}=3 C_{7} / \eta_{0}$ with $\eta_{0}:=(\sqrt{7}-1) / 2$ and $C_{7} \geq 1$ is as in Lemma 5.1, and $C_{9}$ is as in Lemma 5.2. Note that $\frac{\sup f_{\sigma}}{\inf f_{\sigma}}<\infty$ for $|\sigma|$ small (see Remark 3.2).

Remark 2.2. Assumption (2.5) is trivially satisfied if $F$ is Markov. For many one-parameter families of non-Markov AFU maps, one can show that (2.5) only fails at a parameter set of Hausdorff dimension $<1$. This follows from the shrinking targets results [2, Theorem 1 and Corollary 1] and includes the family of shifted $\beta$-transformations $x \mapsto \beta x+\alpha \bmod 1$.

Throughout we fix $k \geq 2 k_{1}$ sufficiently large to satisfy:

$$
\rho^{k}(\rho-1)>12 N_{1} C_{8},
$$

(Inequality (2.6) will be used in estimates in Section 5.) Furthermore, we assume that

$$
\rho^{-2 k}\left(\sup f_{0}+\operatorname{Var} f_{0}\right)\left(\frac{1}{\inf f_{0}}+\operatorname{Var}\left(\frac{1}{f_{0}}\right)\right)<1,
$$

where $f_{0}$ is the positive eigenfunction of $\mathcal{L}_{0}$ associated to eigenvalue $\lambda_{0}=1$.

\subsection{UNI condition restricted to atoms of the image partition $\mathcal{P}_{k}$}

Fix $k$ as in Subsection 2.6. Let $C_{2}^{\prime}:=\frac{C_{2} \rho_{0}}{\rho_{0}-1}$ and $C_{10}:=\left(C_{1} e^{C_{1}}+2\left(1+\varepsilon_{0}\right) e^{\varepsilon_{0} C_{2}^{\prime}} C_{2}^{\prime}+2 C_{6}\right) /\left(2 \eta_{0}-\right.$ $\left.4 \rho_{0}^{-k}\right)$, where it follows from (2.6) that the denominator $2 \eta_{0}-4 \rho_{0}^{-k}>0$. We assume that there exist $D>0$ and a multiple $n_{0}$ of $k$ such that both

$$
C_{10} \rho_{0}^{-n_{0}} \frac{4 \pi}{D} \leq \frac{1}{4}\left(2-2 \cos \frac{\pi}{12}\right)^{1 / 2},
$$

and the UNI (uniform non-integrability) condition holds:

$$
\forall \text { atom } p \in \mathcal{P}_{k}, \exists h_{1}, h_{2} \in \mathcal{H}_{n_{0}} \text { such that } \inf _{x \in p}\left|\psi^{\prime}(x)\right| \geq D,
$$

for $\psi=\varphi_{n_{0}} \circ h_{1}-\varphi_{n_{0}} \circ h_{2}: p \rightarrow \mathbb{R}$.

\subsection{Main result}

Let $b \in \mathbb{R}$. For the class of BV functions we define

$$
\|v\|_{b}=\frac{\operatorname{Var}_{Y} v}{1+|b|}+\|v\|_{1} .
$$

With the above specified, we can state our main result, a Dolgopyat type inequality.

Theorem 2.3. Suppose that all the above assumptions, (2.1) - (2.9), on the AFU map F, on $k$ and on the roof function $\varphi$ hold (in particular, we assume that UNI (2.9) hold for some $D>0$ ). Then there exists $A \geq n_{0}$ and $\varepsilon, \gamma<1$ such that for all $|\sigma|<\varepsilon$ and $|b|>\max \{4 \pi / D, 2\}$ and for all $n \geq A \log |b|$,

$$
\left\|\mathcal{L}_{s}^{n}\right\|_{b} \leq \gamma^{n}
$$

An immediate consequence of the above result (see, for instance, [4]) is 
Corollary 2.4. Suppose that all the above assumptions, (2.1) - (2.9), on the AFU map F, on $k$ and on the roof function $\varphi$ hold. For every $0<\alpha<1$ there exists $\varepsilon \in(0,1)$ and $b_{0}>0$ such that for all $|b| \geq b_{0}$ and for all $|\sigma|<\varepsilon$,

$$
\left\|\left(I-\mathcal{L}_{s}\right)^{-1}\right\|_{b} \leq|b|^{\alpha} .
$$

Remark 2.5. A similar, but simplified, argument (obtained by taking $\sigma=0$ throughout the proof of Theorem 2.3 in this paper) shows that without assuming condition (2.4) (that guarantees exponential tail for the roof function $\varphi$ ) and with no restriction on the class of $B V$ functions, one obtains that for every $0<\alpha<1$, there exists $b_{0}>0$ such that for all $|b| \geq b_{0}$, $\left\|\left(I-\mathcal{L}_{i b}\right)^{-1}\right\|_{b} \leq|b|^{\alpha}$. Of course, this type of inequality does not imply exponential decay of correlation for suspension semiflows, but we believe it to be useful when proving sharp mixing rates for $B V$ observables in the non exponential situation via renewal type arguments (such as sharp bounds for polynomial decay of correlation).

\subsection{Application to suspension semi-flows}

Corollary 2.4 can be used to obtain exponential decay of correlations in terms of BV functions for suspension semiflows over AFU maps with a $C^{1}$ roof function. Let $Y^{\varphi}:=\{(y, u) \in Y \times R$ : $0 \leq u \leq R(y)\} / \sim$, where $(y, \varphi(y)) \sim(F y, 0)$, be the suspension over $Y$. The suspension semiflow $F_{t}: Y^{\varphi} \rightarrow Y^{\varphi}$ is defined by $F_{t}(y, u)=(y, u+t)$ computed modulo identifications. The probability measure $\mu^{\varphi}:=(\mu \times L e b) / \bar{\varphi}$, where $\bar{\varphi}:=\int_{Y} \varphi d \mu$ is $F_{t}$-invariant.

Class of observables Let $F_{\mathrm{BV}, m}\left(Y^{\varphi}\right)$ be the class of observables consisting of $v(y, u)$ : $Y^{\varphi} \rightarrow \mathbb{C}$ such that $v$ is $\operatorname{BV}(Y)$ in $y$ and $C^{m}$ in $u$, so $\|v\|_{\mathrm{BV}, m}:=\sum_{j=0}^{m}\left\|\partial_{t}^{j} v\right\|_{\mathrm{BV}}<\infty$.

For $v \in L^{1}\left(Y^{\varphi}\right)$ and $w \in L^{\infty}\left(Y^{\varphi}\right)$ define the correlation function

$$
\rho_{t}(v, w):=\int_{Y^{\varphi}} v w \circ F_{t} d \mu^{\varphi}-\int_{Y^{\varphi}} v d \mu^{\varphi} \int_{Y^{\varphi}} w d \mu^{\varphi} .
$$

The result below gives exponential decay of correlation for $v \in F_{\mathrm{BV}, 2}\left(Y^{\varphi}\right)$ and $w \in L^{\infty}\left(Y^{\varphi}\right)$. It is likely that this also follows by reinducing $F$ to a Gibbs-Markov AFU map, to which $[4,1]$ apply, together with an approximation argument of BV functions by $C^{2}$ functions. However, it is worthwhile to have the argument for the original map $F$, for instance in situations where reinducing is problematic, such as for families of open AFU maps with shrinking holes.

Theorem 2.6. Suppose that all the above assumptions, (2.1) - (2.9), on the AFU map F and the roof function $\varphi$ hold. Then there exist constants $a_{0}, a_{1}>0$ such that

$$
\left|\rho_{t}(v, w)\right| \leq a_{0} e^{-a_{1} t}\|v\|_{B V, 2}\|w\|_{\infty},
$$

for all $v \in F_{B V, 2}\left(Y^{\varphi}\right)$ and $w \in L^{\infty}\left(Y^{\varphi}\right)$.

The proof of Theorem 2.6 is given in Appendix D. Corollary 2.4 also implies exponential decay of correlations in terms of $\mathrm{BV}$ functions for skew products on $\mathbb{T}^{2}$ as considered in $[6,8]$. We note, however, that the strength of Corollary 2.4 is not needed in the set-up of $[6,8]$ as, in those works, the roof function is bounded and one can restrict the calculations to the imaginary axis.

\section{Twisted and normalized twisted transfer operators}

We start with the continuty of operator $\mathcal{L}_{s}$ in BV.

Proposition 3.1. Let $\varepsilon_{0}>0$ and $C_{3}<\infty$ be as in (2.4). Then there exists $C>0$ and $\varepsilon \in\left(0, \varepsilon_{0}\right)$ such that for all $\left|\sigma_{1}\right|,\left|\sigma_{2}\right|<\varepsilon$ and for all $\left|b_{1}\right|,\left|b_{2}\right| \leq 1,\left\|\mathcal{L}_{\sigma_{1}+i b_{1}}-\mathcal{L}_{\sigma_{2}+i b_{2}}\right\|_{B V} \leq C \varepsilon_{0}^{-1}\left|\sigma_{1}-\sigma_{2}\right|$. 
The proof of Proposition 3.1 is deferred to the end of Appendix A.

Remark 3.2. An immediate consequence of Proposition 3.1 is that for any $\delta \in(0,1)$, there exists $\varepsilon \in(0,1)$ such that

$$
\sup _{|\sigma|<\varepsilon}\left|\lambda_{\sigma}-1\right|<\delta, \quad \sup _{|\sigma|<\varepsilon}\left\|\frac{f_{\sigma}}{f_{0}}-1\right\|_{B V}<\delta, \quad \sup _{|\sigma|<\varepsilon}\left\|\frac{f_{\sigma}}{f_{0}}-1\right\|_{\infty}<\delta
$$

for all $|\sigma|<\varepsilon$. Recall that $\frac{1}{C_{4}} \leq f_{0}(x) \leq C_{4}$ for all $x \in Y$. It follows that $\frac{f_{\sigma}(x)}{f_{\sigma}(y)}=$ $\frac{f_{\sigma}(x)}{f_{0}(x)} \frac{f_{0}(x)}{f_{0}(y)} \frac{f_{0}(y)}{f_{\sigma}(y)} \leq(1+\delta) C^{2}(1-\delta)^{-1}<\infty$ for all $x, y \in Y$. Hence, $\frac{\sup f_{\sigma}}{\inf f_{\sigma}} \leq C_{5}$ for $C_{5}:=\frac{1+\delta}{1-\delta} C_{4}^{2}$ and $|\sigma|<\varepsilon$.

Since $\lambda_{0}=1$ and $f_{0}$ is strictly positive, due to the continuity of $\lambda_{\sigma}$ and $f_{\sigma}$ in $\sigma$, we can ensure that for $\varepsilon>0$ sufficiently small

$$
\rho^{-1 / 4}<\lambda_{\sigma} \text { and } f_{\sigma} \text { is strictly positive for all }|\sigma|<\varepsilon .
$$

By assumption (2.7) and Remark 3.2, we can choose $\varepsilon$ small enough such that for all $|\sigma|<\varepsilon$,

$$
\rho^{-2 k}\left(\sup f_{\sigma}+\operatorname{Var} f_{\sigma}\right)\left(\frac{1}{\inf f_{\sigma}}+\operatorname{Var}\left(\frac{1}{f_{\sigma}}\right)\right)<1 .
$$

(The above formula will be used in the proof of Proposition 3.5.)

Lemma 3.3. There exists $\varepsilon \in(0,1)$ so small that for all $|\sigma|<\varepsilon$ and for all $n \geq 1$,

$$
\frac{1}{\lambda_{\sigma}^{n}} \sup _{h \in \mathcal{H}_{n}} \sup _{x \in \operatorname{dom}(h)}\left|h^{\prime}(x)\right| e^{\sigma \varphi_{n} \circ h(x)} \leq \rho^{-3 n} .
$$

Remark 3.4. Without assumption (2.4) (i.e., without the exponential tail assumption), we still have

$$
\sup _{h \in \mathcal{H}_{n}} \sup _{x \in \operatorname{dom}(h)}\left|h^{\prime}(x)\right| e^{\sigma \varphi_{n} \circ h(x)} \leq \rho^{-3 n}
$$

for $-\varepsilon<\sigma \leq 0$.

Proof. We start with $n=1$. By continuity of $\lambda_{\sigma}$, we can take $\varepsilon$ so small that $\lambda_{\sigma}^{4 u} \rho_{0}^{u-1}>C_{3}$ for $u=\left\lfloor\varepsilon_{0} /(4 \varepsilon)\right\rfloor$ with $\varepsilon_{0} \in(0,1)$ and $C_{3}$ such that (2.4) hold. For $h \in \mathcal{H}_{1}$ assume by contradiction that $\lambda_{\sigma}^{-1}\left|h^{\prime}(x)\right| e^{\sigma \varphi \circ h(x)}>\rho^{-3}$ for some $x \in \operatorname{dom}(h)$. Since $\left|h^{\prime}\right| \leq \rho_{0}^{-1}=\rho^{-4}$ we have

$$
\lambda_{\sigma}^{-1} e^{\sigma \varphi \circ h(x)} \geq \lambda_{\sigma}^{-1} \rho^{4}\left|h^{\prime}\right| e^{\sigma \varphi \circ h(x)}>\rho=\rho_{0}^{1 / 4} \geq\left|h^{\prime}\right|^{-1 / 4} .
$$

Therefore,

$$
\begin{aligned}
\left|h^{\prime}\right| e^{\varepsilon_{0} \varphi \circ h} & >\left|h^{\prime}\right| e^{4 u \varepsilon \varphi \circ h} \geq\left|h^{\prime}\right| e^{4 u \sigma \varphi \circ h} \geq\left|h^{\prime}\right|\left(\lambda_{\sigma}^{-1} e^{\sigma \varphi \circ h}\right)^{4 u} \lambda_{\sigma}^{4 u} \\
& \geq\left|h^{\prime}\right|^{1-u} \lambda_{\sigma}^{4 u} \geq \rho_{0}^{u-1} \lambda_{\sigma}^{4 u} \geq C_{3}
\end{aligned}
$$

contradicting (2.4). The statement for $n \geq 1$ follows immediately.

Let

$$
\tilde{\mathcal{L}}_{s} v=\frac{1}{\lambda_{\sigma} f_{\sigma}} \mathcal{L}_{s}\left(f_{\sigma} v\right) \quad \text { and } \quad \tilde{\mathcal{L}}_{\sigma} v=\frac{1}{\lambda_{\sigma} f_{\sigma}} \mathcal{L}_{\sigma}\left(f_{\sigma} v\right)
$$

be the normalized versions of $\mathcal{L}_{s}$ and $\mathcal{L}_{\sigma}$.

Proposition 3.5 (Lasota-Yorke type inequality). Choose $k$ and $\varepsilon_{1} \in(0,1)$ such that (3.2) and (3.3) hold. Define $\Lambda_{\sigma}=\lambda_{2 \sigma}^{1 / 2} / \lambda_{\sigma}$. Then, there exist $\varepsilon \leq \varepsilon_{1}, \rho>1$ and $c>0$ such that for all $s=\sigma+i b$ with $|\sigma|<\varepsilon$ and $b \in \mathbb{R}$,

$$
\operatorname{Var}_{Y}\left(\tilde{\mathcal{L}}_{s}^{n k} v\right) \leq \rho^{-n k} \operatorname{Var}_{Y} v+c(1+|b|) \Lambda_{\sigma}^{n k}\left(\|v\|_{\infty}\|v\|_{1}\right)^{1 / 2} .
$$

for all $v \in B V(Y)$ and all $n \geq 1$. 
Proposition 3.5 would be meaningless if $\Lambda_{\sigma}<1$, but one can check that $1 \leq \Lambda_{\sigma}=1+O\left(\sigma^{2}\right)$. The proof of Proposition 3.5 is deferred to Appendix A.

In what follows we focus on the controlling the term containing $\left(\|v\|_{\infty}\|v\|_{1}\right)^{1 / 2}$ and proceed as in [4]: we estimate the $L^{2}$ norm of $\tilde{\mathcal{L}}_{s}^{n}$ for $n$ large enough. Once we obtain a good estimate for the $L^{2}$ norm, we combine it with the estimate in Proposition 3.5 (following the pattern in $[1,3,4]$ ) to prove Theorem 2.3.

\section{New ingredients of the proof}

The basic strategy of the proof using the cancellation lemma follows [1, 3, 4]. For the non-Markov AFU maps, we use the space BV, and hence observables $u, v \in \mathrm{BV}$ can have jumps. The task is to locate and control the sizes of these jumps. Given a discontinuity point $x$ for a function $v$, we define the size of the jump at $x$ as

$$
\text { Size } v(x)=\lim _{\delta \rightarrow 0} \sup _{\xi, \xi^{\prime} \in(x-\delta, x+\delta)}\left|v(\xi)-v\left(\xi^{\prime}\right)\right| .
$$

Recall that the oscillation of a function $v: I \rightarrow \mathbb{C}$ on a subinterval $I \subset Y$ is defined as

$$
\operatorname{Osc}_{I} v=\sup _{\xi, \xi^{\prime} \in I}\left|v(\xi)-v\left(\xi^{\prime}\right)\right|
$$

It follows that

$$
\operatorname{Osc}_{I} v \leq \operatorname{Osc}_{I^{\circ}} v+\operatorname{Size} v(x)+\operatorname{Size} v(y)
$$

for $I=[x, y]$ with interior $I^{\circ}$. For positive functions, (4.1) reduces to

$$
\text { Size } u(x)=\limsup _{\xi \rightarrow x} u(\xi)-\liminf _{\xi \rightarrow x} u(\xi)=\left|\lim _{\xi \uparrow x} u(\xi)-\lim _{\xi \downarrow x} u(\xi)\right| \text {. }
$$

We adopt the convention $u(x)=\lim \sup _{\xi \rightarrow x} u(\xi)$ at discontinuity points, so we always have the trivial inequality Size $u(x) \leq u(x)$.

Definition 4.1. Let $k \geq 1$ such that (2.5) holds and take $C_{7}$ as in Lemma 5.1. We say that a pair of functions $u, v \in B V(Y)$ with $|v| \leq u$ and $u>0$ has exponentially decreasing jump-sizes, if the discontinuities of $u$ and $v$ belong to $X_{\infty}=\cup_{j \geq 1} X_{j}^{\prime}$ and if $x \in X_{j}^{\prime}$ for $j>k$ is such a discontinuity, then

$$
\text { Size v }(x) \text {, Size } u(x) \leq C_{7} \rho^{-j} u(x) .
$$

Example 4.2. For the reader's convenience, we provide a simple example of functions $(u, v)$ with exponentially decreasing jump-sizes. Assume that $Y=[p, q]$. Let $\left\{a_{i}\right\}_{i \geq 1}$ be a sequence in $\mathbb{C}$ such that $\left|a_{i}\right| \rightarrow 0$ exponentially fast, and $\left\{x_{i}\right\}_{i \geq 1} \subset[p, q]$. Then

$$
v=\sum_{i \geq 1} a_{i} 1_{\left[x_{i}, q\right]} \quad u=\sum_{i \geq 1}\left|a_{i}\right| 1_{\left[x_{i}, q\right]}
$$

is a pair of functions having exponentially decreasing jump-sizes when $X_{j}^{\prime}=\left\{x_{j}\right\}$. Indeed, let $\delta^{\prime}>0$ be arbitrary and let $N \in \mathbb{N}$ be such that $\sum_{i>N}\left|a_{i}\right| \leq \delta^{\prime}$. Assuming for simplicity that the $x_{i}$ are distinct, we have

$$
\begin{aligned}
\text { Size } v\left(x_{j}\right) & =\lim _{\delta \rightarrow 0} \sup _{\xi, \xi^{\prime} \in\left(x_{j}-\delta, x_{j}+\delta\right)}\left|\sum_{i \geq 1} a_{i}\left(1_{\left[x_{i}, q\right]}(\xi)-1_{\left[x_{i}, q\right]}\left(\xi^{\prime}\right)\right)\right| \\
& \leq \lim _{\delta \rightarrow 0} \sup _{\xi, \xi^{\prime} \in\left(x_{j}-\delta, x_{j}+\delta\right)}\left|\sum_{i=1}^{N} a_{i}\left(1_{\left[x_{i}, q\right]}(\xi)-1_{\left[x_{i}, q\right]}\left(\xi^{\prime}\right)\right)\right|+\delta^{\prime}=\left|a_{j}\right|+\delta^{\prime} .
\end{aligned}
$$

Since $\delta^{\prime}$ was arbitrary, Size $v\left(x_{j}\right) \leq\left|a_{j}\right|$. So, Size $v\left(x_{j}\right)$ is exponentially small in $j$. On the other hand, if $x \notin\left\{x_{i}\right\}_{i \in \mathbb{N}}$, then $v$ is continuous at $x$, so Size $v(x)=0$. A similar computation holds for Size $u\left(x_{j}\right)$. 
Definition 4.1 states that the discontinuities of $(u, v)$ can only appear in $X_{\infty}:=\cup_{j \geq 1} X_{j}^{\prime}$, and we will see in Proposition 5.3 that this property is preserved under $(u, v) \mapsto\left(\tilde{\mathcal{L}}_{\sigma}^{n} u, \tilde{\mathcal{L}}_{s}^{n} v\right)$. For a given $n$, we will distinguish between two types of discontinuities of $\tilde{\mathcal{L}}_{\sigma}^{n} u$.

(i) Created discontinuities. In this case $x \in \partial \operatorname{dom}(h)$ for some $h \in \mathcal{H}_{n}$ and $x \in X_{j}^{\prime}$ for some $1 \leq j \leq n$. The discontinuity is created because the sum $\sum_{h \in \mathcal{H}, \xi \in \operatorname{dom}(h)}$ involved in $\tilde{\mathcal{L}}_{\sigma}^{n} u$ runs over a different collection of inverse branches depending on whether $\xi$ is close to the left or close to the right of $x$ : in only one of the cases $h$ is part of this collection. It is not important whether the function $u$ is continuous at $y=h(x)$.

(ii) Propagated discontinuities. Here the function $u: Y \rightarrow \mathbb{R}_{+}$has discontinuities. Hence, it is discontinuous at $y=h(x)$ for some $h \in \mathcal{H}_{n}$. In this case $y \in X_{j}^{\prime}$ for some $j \geq 1$ and hence $x \in X_{j+n}^{\prime}$.

Consequently, we define a cone $\mathcal{C}_{b}$ of $\mathrm{BV}$ functions with discontinuities of the type prescribed in Definition 4.1. In Appendix B, we prove that the eigenfunction $f_{\sigma}$ and $1 / f_{\sigma}$ belong to $\mathcal{C}_{b}$. This argument is independent of Section 7 where the invariance of $\mathcal{C}_{b}$ under the transformation $(u, v) \mapsto\left(\tilde{\mathcal{L}}_{\sigma}^{n}(\chi u), \tilde{\mathcal{L}}_{s}^{n} v\right)$ is proved. This invariance depends crucially on Proposition 5.3, which together with an inductive bound on $\frac{\left.\sup u\right|_{p}}{\left.\inf u\right|_{p}}$ for $p \in \mathcal{P}_{k}$ and assumption (2.5) imply that discontinuities indeed behave as outlined in this section. To deal with BV observables $v \notin \mathcal{C}_{b}$, we exploit the fact that the size of discontinuities at points $x \notin X_{\infty}$ decrease exponentially under iteration of $\tilde{\mathcal{L}}_{s}$. This means that $\tilde{\mathcal{L}}_{s}^{n} v$ converges exponentially fast to $\mathcal{C}_{b}$ and this suffices to prove the results for arbitrary BV observables.

\section{Towards the cone condition: discontinuities and jump-sizes}

Recall the sets $X_{j}^{\prime}$ from Section 2.4 and let $k$ satisfy the conditions in Subsection 2.6. To deal with the discontinuities of $(u, v)$, we introduce the "extra term" for intervals $I \subset Y$ :

$$
E_{I}(u):=\sum_{j>k} \rho^{-j} \sum_{x \in X_{j}^{\prime} \cap I^{\circ}} \limsup _{\xi \rightarrow x} u(\xi)
$$

where we recall that $\# X_{j}^{\prime} \leq N_{1}$ for all $j \geq 1$. The choice of $k$ in (2.6) implies that $C_{8} E_{I}(u) \leq$ $\frac{1}{12} \sup _{I} u$ for every $I$ contained in a single atom of $\mathcal{P}_{k}$.

Throughout this and the next section we set $n=2 k$. We start with two lemmas on the properties of the eigenfunction $f_{\sigma}$, which will be proved in Section B. We recall (see Remark 1.4) that $f_{\sigma}$ is the positive eigenfunction of $\mathcal{L}_{\sigma}$ with eigenvalue $\lambda_{\sigma}$.

Lemma 5.1. There are $C_{6}, C_{7} \geq 1$ such that for all $\sigma$ with $|\sigma|<\varepsilon$ the following holds:

1. $f_{\sigma}$ has discontinuities only in $X_{\infty}$, and if $x_{j} \in X_{j}^{\prime}$, then Size $f_{\sigma}\left(x_{j}\right) \leq C_{7} \rho^{-3 j} \sup f_{\sigma}$.

2. For every interval $I \subset Y$ we have

$$
\operatorname{Osc}_{I^{\circ}}\left(f_{\sigma}\right) \leq C_{6} \operatorname{Leb}(I) \inf _{I} f_{\sigma}+C_{7} E_{I}\left(f_{\sigma}\right) \text { and } \operatorname{Osc}_{I^{\circ}}\left(\frac{1}{f_{\sigma}}\right) \leq C_{6} \operatorname{Leb}(I) \inf _{I} \frac{1}{f_{\sigma}}+C_{7} E_{I}\left(\frac{1}{f_{\sigma}}\right) \text {. }
$$

Lemma 5.2. Choose $k$ such that (2.5) holds and set $n=2 k$. Then there exists $\varepsilon \in(0,1)$ and $C_{9} \in(0,1)$ such that

$$
\lambda_{\sigma}^{-n} \inf _{x \in Y} \sum_{\substack{\in \in \mathcal{H}, n, x \in \operatorname{dom}(h) \\ \operatorname{range}(h) \subset p}}\left|h^{\prime}(x)\right| e^{\sigma \varphi_{n} \circ h(x)} \geq C_{9} \operatorname{Leb}(p)
$$

for all $p \in \mathcal{P}_{k}$ and $|\sigma|<\varepsilon$.

The main result in this section is the following.

Proposition 5.3. Choose $k$ such that (2.5) holds and set $n=2 k$. If the pair $(u, v)$ with $|v| \leq u$ has exponentially decreasing jump-sizes (4.4), then for each $x \in X_{j}^{\prime}$ with $j>k$, we have

$$
\text { Size } \tilde{\mathcal{L}}_{\sigma}^{n} u(x) \text {, Size } \tilde{\mathcal{L}}_{s}^{n} v(x) \leq \frac{1}{4} \max _{p \in \mathcal{P}_{k}} \frac{\left.\sup u\right|_{p}}{\left.\inf u\right|_{p}} C_{7} \rho^{-j} \tilde{\mathcal{L}}_{\sigma}^{n} u(x) .
$$


Remark 5.4. It is possible that $x$ belongs to different $X_{j}^{\prime}$ 's at the same time. This means that the discontinuity at $x$ is propagated by different branches of $F$ ( or $x \in X_{1}^{\prime} \cap X_{j}^{\prime}$ for some $j \geq 2$, and the discontinuity at $x$ is generated in $\mathcal{P}_{1}$ as well as propagated from another discontinuity at some point in $\left.X_{j-1}^{\prime}\right)$. In this case, we add the jump-sizes at $x$ but the proof remains the same, i.e., writing $x=x_{j}=x_{j^{\prime}}$ for $x_{j} \in X_{j}^{\prime}$ and $x_{j^{\prime}} \in X_{j^{\prime}}^{\prime}$, Size $v(x)=\operatorname{Size} v\left(x_{j}\right)+\operatorname{Size} v\left(x_{j^{\prime}}\right) \leq$ $C_{7}\left(\rho^{-j}+\rho^{-j^{\prime}}\right)\|u\|_{\infty}$.

Proof of Proposition 5.3. By Lemma 5.1, we know that $f_{\sigma}$ and $1 / f_{\sigma}$ have exponentially decreasing jump-sizes with parameters $C_{7}$ and $\rho^{3}$.

Let $y=\tilde{h}(x)$ for some $\tilde{h} \in \mathcal{H}_{r}$ and $r>k$ to be determined below. Let $p \in \mathcal{P}_{k}$ such that $y \in \bar{p}$. Then

$$
\begin{aligned}
\tilde{\mathcal{L}}_{\sigma}^{r} u(x) & \geq \frac{1}{\lambda_{\sigma}^{r} f_{\sigma}(x)} \sum_{\substack{h \in \mathcal{H}_{r} \\
\operatorname{range}(h) \subset p}}\left|h^{\prime}\right| e^{\sigma \varphi_{r} \circ h(x)}\left(f_{\sigma} u\right) \circ h(x) \\
& \geq \frac{\inf f_{\sigma}}{f_{\sigma}(x)} \frac{\left.\inf u\right|_{p}}{\left.\sup u\right|_{p}} u(y) \lambda_{\sigma}^{-r} \sum_{\substack{h \in \mathcal{H}_{r}, x \in \operatorname{dom}(h) \\
\text { range }(h) \subset p}}\left|h^{\prime}(x)\right| e^{\sigma \varphi_{r} \circ h(x)} \\
& \geq \frac{\inf f_{\sigma}}{f_{\sigma}(x)} \frac{\left.\inf u\right|_{p}}{\left.\sup u\right|_{p}} C_{9} \operatorname{Leb}(p) u(y)
\end{aligned}
$$

by Lemma 5.2.

First take $j>n$ and $x \in X_{j}^{\prime}$, so $x$ is a discontinuity propagated from some $y \in X_{j-n}^{\prime}$. Let $\tilde{h} \in \mathcal{H}_{n}$ such that $\tilde{h}(x)=y$ be the corresponding inverse branch. This is the only inverse branch that contributes to Size $\tilde{\mathcal{L}}_{s}^{n} v(x)$. We compute using (3.3) and Lemma 5.1,

$$
\begin{aligned}
& \text { Size } \tilde{\mathcal{L}}_{s}^{n} v(x)=\operatorname{Size}\left(\left|\tilde{h}^{\prime}\right| e^{s \varphi_{n} \circ \tilde{h}} \frac{\left(f_{\sigma} v\right) \circ \tilde{h}}{\lambda_{\sigma}^{n} f_{\sigma}}\right)(x) \\
& \leq \quad \frac{1}{\lambda_{\sigma}^{n}}\left|\tilde{h}^{\prime}(x)\right| e^{\sigma \varphi_{n} \circ \tilde{h}(x)}\left(\frac{|v(y)|}{f_{\sigma}(x)} \operatorname{Size} f_{\sigma}(y)+f_{\sigma}(y)|v(y)| \operatorname{Size} \frac{1}{f_{\sigma}}(x)+\frac{f_{\sigma}(y)}{f_{\sigma}(x)} \operatorname{Size} v(y)\right) \\
& \leq \quad 4 \rho^{-3 n} \frac{\sup f_{\sigma}}{f_{\sigma}(x)} u(y) \times \begin{cases}C_{7} \rho^{-(j-n)} & \text { if } j-n>k, \\
1 & \text { if } j-n \leq k .\end{cases}
\end{aligned}
$$

This distinction is because (4.4) only holds for $j-n>k$; for $j-n \leq k$ we only have the trivial bound Size $v(y) \leq u(y)$. The factor 4 is to account for the three terms in the penultimate line above; in particular, Size $v(y) \leq 2 u(y)$, so the factor 4 appears despite the presence of just three terms. Since $\rho^{-2 n} \leq \rho^{-4 k}$, we have

$$
\text { Size } \tilde{\mathcal{L}}_{s}^{n} v(x) \leq \frac{4 \sup f_{\sigma}}{\rho^{3 k} f_{\sigma}(x)} C_{7} \rho^{-j} u(y)
$$

in either case.

Combining (5.4) and (5.2) for $y=\tilde{h}(x)$ and $r=n$, and using the bound on $\operatorname{Leb}(p)$ from (2.5) we obtain

$$
\text { Size } \tilde{\mathcal{L}}_{s}^{n} v(x) \leq \frac{4 C_{7}}{C_{9} \rho^{3 k} \operatorname{Leb}(p)} \frac{\left.\sup u\right|_{p}}{\left.\inf u\right|_{p}} \frac{\sup f_{\sigma}}{\inf f_{\sigma}} \rho^{-j} \tilde{\mathcal{L}}_{\sigma}^{n} u(x) \leq \frac{1}{4} \frac{\left.\sup u\right|_{p}}{\left.\inf u\right|_{p}} C_{7} \rho^{-j} \tilde{\mathcal{L}}_{\sigma}^{n} u(x) .
$$

Now take $k<j \leq n$, so the discontinuity at $x \in X_{j}^{\prime}$ is created by non-onto branches of $F^{n}$, and there exist $y \in X_{1}^{\prime}$ and an inverse branch $\tilde{h} \in \mathcal{H}_{j-1}$ such that $y=\tilde{h}(x)$. Then, analogous to (5.3),

$$
\begin{aligned}
\text { Size } \tilde{\mathcal{L}}_{s}^{n} v(x) & =\operatorname{Size}\left(\left|\tilde{h}^{\prime}\right| e^{s \varphi_{j-1} \circ \tilde{h}} \frac{\left(f_{\sigma} v\right) \circ \tilde{h}}{\lambda_{\sigma}^{n} f_{\sigma}}\right)(x) \\
& \leq \frac{1}{\lambda_{\sigma}^{n}}\left|\tilde{h}^{\prime}(x)\right| e^{\sigma \varphi_{j-1} \circ \tilde{h}(x)} \frac{4 \sup f_{\sigma}}{f_{\sigma}(x)} u(y) \\
& \leq \frac{\rho^{-3(j-1)}}{\lambda_{\sigma}^{n-j+1}} \frac{4 \sup f_{\sigma}}{f_{\sigma}(x)} u(y) \leq \frac{4 C_{7} \sup f_{\sigma}}{\rho^{k} f_{\sigma}(x)} \rho^{-j} u(y)
\end{aligned}
$$


because $C_{7} \geq 1, k<j \leq n$ and $\lambda_{\sigma}^{-4} \leq \rho$ by (3.1). Combining this with (5.2) to bound $u(y)$ (but applied to $r=j$ ) and (2.5) gives

$$
\text { Size } \tilde{\mathcal{L}}_{s}^{n} v(x) \leq \frac{4 C_{7}}{C_{9} \rho^{k} \operatorname{Leb}(p)} \frac{\left.\sup u\right|_{p}}{\left.\inf u\right|_{p}} \frac{\sup f_{\sigma}}{\inf f_{\sigma}} \rho^{-j} \tilde{\mathcal{L}}_{\sigma}^{n} u(x) \leq \frac{1}{4} \frac{\left.\sup u\right|_{p}}{\left.\inf u\right|_{p}} C_{7} \rho^{-j} \tilde{\mathcal{L}}_{\sigma}^{n} u(x),
$$

as before. The computations for $\tilde{\mathcal{L}}_{\sigma}^{n} u$ are the same.

\section{Cancellation lemma}

We define a cone of function pairs $(u, v)$ :

$$
\begin{aligned}
\mathcal{C}_{b}=\{(u, v): & 0<u, 0 \leq|v| \leq u,(u, v) \text { has exponentially decreasing } \\
& \text { jump-sizes (4.4) and } \operatorname{Osc}_{I} v \leq\left. C_{10}|b| \operatorname{Leb}(I) \sup u\right|_{I}+C_{8} E_{I}(u), \\
& \text { for all intervals } \left.I \text { contained in a single atom of } \mathcal{P}_{k}\right\} .
\end{aligned}
$$

Recall that the choice of $k$ in (2.6) implies that $C_{8} E_{I}(u) \leq \frac{1}{12} \sup _{I} u$ for every $I$ contained in a single atom of $\mathcal{P}_{k}$. In Section 7 we show that $\mathcal{C}_{b}$ is 'invariant' in the sense of [4]: see Lemma 7.1.

In this section we provide a cancellation lemma for pairs of functions in $\mathcal{C}_{b}$ similar to the one in [4]. The statement and proof of Lemma 6.1 below follows closely the pattern of the statements and proofs of [4, Lemma 2.4] and [1, Lemma 2.9]. In this section, we abbreviate

$$
A_{s, h, n}=e^{s \varphi_{n} \circ h}\left|h^{\prime}\right| v \circ h
$$

for $h \in \mathcal{H}_{n}$ and $\varphi_{n}=\sum_{j=0}^{n-1} \varphi \circ F^{j}$.

Lemma 6.1. Fix $k$ such that (2.5) holds. Recall that $\eta_{0}=\frac{\sqrt{7}-1}{2} \in(2 / 3,1)$. Assume that the UNI condition in Subsection 2.7 holds (with constant $D>0, k$ fixed and $n_{0} \geq 1$ ).

Set $\Delta=\frac{2 \pi}{D}$. There exists $\delta \in(0, \Delta)$ such that the following hold for all $|\sigma|<\varepsilon,|b|>2 \Delta$ and for all $(u, v) \in \mathcal{C}_{b}$ :

Let $p \in \mathcal{P}_{k}$ and let $h_{1}, h_{2} \in \mathcal{H}_{n_{0}}$ be the branches from UNI. For every $y_{0} \in p$ there exists $y_{1} \in B_{\Delta /|b|}\left(y_{0}\right)$ such that one of the following inequalities holds on $B_{\delta /|b|}\left(y_{1}\right)$ :

Case $h_{1} .\left|A_{s, h_{1}, n_{0}}\left(f_{\sigma} v\right)+A_{s, h_{2}, n_{0}}\left(f_{\sigma} v\right)\right| \leq \eta_{0} A_{\sigma, h_{1}, n_{0}}\left(f_{\sigma} u\right)+A_{\sigma, h_{2}, n_{0}}\left(f_{\sigma} u\right)$.

Case $h_{2} .\left|A_{s, h_{1}, n_{0}}\left(f_{\sigma} v\right)+A_{s, h_{2}, n_{0}}\left(f_{\sigma} v\right)\right| \leq A_{\sigma, h_{1}, n_{0}}\left(f_{\sigma} u\right)+\eta_{0} A_{\sigma, h_{2}, n_{0}}\left(f_{\sigma} u\right)$.

Proof. Choose $\delta \in(0, \Delta)$ sufficiently small such that

$$
\delta \frac{D}{16 \pi}<\frac{1}{12}, \quad C_{0} \delta<\frac{\pi}{6} .
$$

Let $y_{0} \in Y$. Note that for $m=1,2$,

$$
\sup _{B_{\delta /|b|}\left(y_{0}\right)}\left|v \circ h_{m}\right| \leq \operatorname{Osc}_{B_{\delta /|b|}\left(y_{0}\right)}\left(v \circ h_{m}\right)+\inf _{B_{\delta /|b|}\left(y_{0}\right)}\left|v \circ h_{m}\right|+\operatorname{Size} v\left(B_{\delta /|b|}\left(y_{0}\right)\right) .
$$

Since $(u, v) \in \mathcal{C}_{b}$,

$$
\begin{aligned}
\sup _{B_{\delta /|b|}\left(y_{0}\right)}\left|v \circ h_{m}\right| \leq C_{10} \operatorname{Leb}\left(h_{m}\left(B_{\delta /|b|}\left(y_{0}\right)\right)\right)|b| \sup _{B_{\delta /|b|}\left(y_{0}\right)}\left(u \circ h_{m}\right) & +\inf _{B_{\delta /|b|}\left(y_{0}\right)}\left|v \circ h_{m}\right| \\
& +C_{8} E_{B_{\delta /|b|}\left(y_{0}\right)}(u) .
\end{aligned}
$$

But

$$
C_{10} \operatorname{Leb}\left(h_{m}\left(B_{\delta /|b|}\left(y_{0}\right)\right)\right) \leq C_{10} \rho_{0}^{-n_{0}} \operatorname{Leb}\left(B_{\delta /|b|}\left(y_{0}\right)\right)=C_{10} \rho_{0}^{-n_{0}} \frac{\delta}{|b|} \leq \frac{D}{16 \pi} \frac{\delta}{|b|}
$$


where in the last inequality we have used (2.8). Putting the above together with the estimate on $E_{I}(u)$ below equation (5.1) and using the choice of $\delta$ and $k$,

$$
\sup _{B_{\delta /|b|}\left(y_{0}\right)}\left|v \circ h_{m}\right| \leq \frac{1}{6} \sup _{B_{\delta /|b|}\left(y_{0}\right)}\left(u \circ h_{m}\right)+\inf _{B_{\delta /|b|}\left(y_{0}\right)}\left|v \circ h_{m}\right| .
$$

Case 1. Suppose that $\inf _{B_{\delta /|b|}\left(y_{0}\right)}\left|v \circ h_{m}\right| \leq \frac{1}{2} \sup _{B_{\delta /|b|}\left(y_{0}\right)}\left(u \circ h_{m}\right)$ for $m=1,2$. Then (6.3) implies that

$$
\sup _{B_{\delta /|b|}\left(y_{0}\right)}\left|v \circ h_{m}\right| \leq\left(\frac{1}{2}+\frac{1}{6}\right) \sup _{B_{\delta /|b|}\left(y_{0}\right)}\left(u \circ h_{m}\right)=\frac{2}{3} \sup _{B_{\delta /|b|}\left(y_{0}\right)}\left(u \circ h_{m}\right)<\eta_{0} \sup _{B_{\delta /|b|}\left(y_{0}\right)}\left(u \circ h_{m}\right) .
$$

Thus, for $m=1,2,\left|A_{s, h_{m}, n_{0}}\left(f_{\sigma} v\right)(y)\right| \leq \eta_{0} A_{\sigma, h_{m}, n_{0}}\left(f_{\sigma} u\right)(y)$ for all $y \in B_{\delta /|b|}\left(y_{0}\right)$. So, Case $h_{m}$ holds with $y_{1}=y_{0}$.

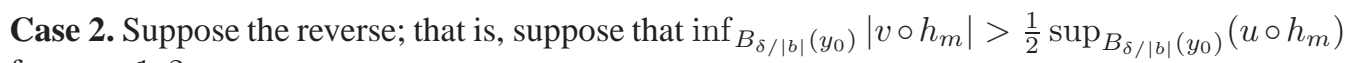
for $m=1,2$.

For $m=1,2$, write $A_{s, h_{m}, n_{0}}\left(f_{\sigma} v\right)(y)=r_{m}(y) e^{i \theta_{m}(y)}$. Let $\theta(y)=\theta_{1}(y)-\theta_{2}(y)$. Choose $\delta$ as in (6.2) and recall $\Delta=\frac{2 \pi}{D}$. A calculation [4, Lemma 2.3] shows that if $\cos \theta \leq 1 / 2$ then $r_{1} e^{i \theta_{1}}+r_{2} e^{i \theta_{2}} \leq \max \left\{\eta_{0} r_{1}+r_{2}, r_{1}+\eta_{0} r_{2}\right\}$. Thus, the conclusion follows once we show that $\cos \theta(y) \leq 1 / 2$, or equivalently $|\theta(y)-\pi|<2 \pi / 3$, for all $y \in B_{\delta /|b|}\left(y_{1}\right)$ for some $y_{1} \in B_{\Delta /|b|}\left(y_{0}\right)$. In what follows we show that $\left|\sup _{B_{\delta /|b|}\left(y_{1}\right)} \theta-\pi\right|<2 \pi / 3$, for some $y_{1} \in B_{\Delta /|b|}\left(y_{0}\right)$.

We start by restricting to $B_{\xi /|b|}\left(y_{0}\right)$, where $\xi=\delta+\Delta$. Note that $\theta=V-b \psi$, where $\psi=\psi_{h_{1}, h_{2}}$ is the quantity defined in UNI and $V=\arg \left(v \circ h_{1}\right)-\arg \left(v \circ h_{2}\right)$. We first estimate $\operatorname{Osc}_{B_{\xi /|b|}\left(y_{0}\right)} V$. For this purpose, we recall a basic trigonometry result (also used in in [4] and [1]): if $\left|z_{1}\right|,\left|z_{2}\right| \geq c$ and $\left|z_{1}-z_{2}\right| \leq c(2-2 \cos \omega)^{1 / 2}$ for $c>0$ and $|\omega|<\pi$ then $\left|\arg \left(z_{1}\right)-\arg \left(z_{2}\right)\right| \leq$ $\omega$.

Since $(u, v) \in \mathcal{C}_{b}$ and $\xi<4 \pi / D$ for $m=1,2$, we have by (2.8)

$$
\begin{aligned}
\operatorname{Osc}_{B_{\xi /|b|}\left(y_{0}\right)}\left(v \circ h_{m}\right) & \leq C_{10} \rho_{0}^{-n_{0}} \frac{4 \pi}{D} \sup _{B_{\xi /|b|}\left(y_{0}\right)}\left(u \circ h_{m}\right) \\
& \leq \frac{1}{4}\left(2-2 \cos \frac{\pi}{12}\right)^{1 / 2} \sup _{B_{\xi /|b|}\left(y_{0}\right)}\left(u \circ h_{m}\right) .
\end{aligned}
$$

Recalling the assumption of Case 2,

$$
\begin{aligned}
\sup _{B_{\xi /|b|}\left(y_{0}\right)}\left|v \circ h_{m}\right| & \geq\left|\sup _{B_{\xi /|b|}\left(y_{0}\right)}\right| v \circ h_{m}\left|-\operatorname{Osc}_{B_{\xi /|b|}\left(y_{0}\right)}\left(v \circ h_{m}\right)\right| \\
& \geq \frac{1}{2} \sup _{B_{\delta /|b|}\left(y_{0}\right)}\left(u \circ h_{m}\right)-\frac{1}{4} \sup _{B_{\xi /|b|}\left(y_{0}\right)}\left(u \circ h_{m}\right)=\frac{1}{4} \sup _{B_{\xi /|b|}\left(y_{0}\right)}\left(u \circ h_{m}\right) .
\end{aligned}
$$

By equations (6.4) and (6.5),

$$
\sup _{z_{1}, z_{2} \in B_{\delta /|b|}\left(y_{0}\right)}\left|\arg \left(v \circ h_{m}\left(z_{1}\right)\right)-\arg \left(v \circ h_{m}\left(z_{2}\right)\right)\right| \leq \frac{\pi}{12},
$$

and thus

$$
\operatorname{Osc}_{B_{\xi /|b|}\left(y_{0}\right)} V \leq \frac{\pi}{6}
$$

Next, recall the UNI assumption in Subsection 2.7. Note that for any $z \in B_{\Delta /|b|}\left(y_{0}\right)$,

$$
|b(\psi(z)-\psi(y))| \geq|b|\left|z-y_{0}\right| \inf \left|\psi^{\prime}\right| \geq D|b|\left|z-y_{0}\right|=\frac{2 \pi}{\Delta}|b|\left|z-y_{0}\right| .
$$


Since $|b|>2 \Delta$, the ball $B_{\Delta /|b|}\left(y_{0}\right) \subset Y$ contains an interval of length at least $\Delta /|b|$. Hence, as $z$ varies in $B_{\Delta /|b|}\left(y_{0}\right)$, it fills out an interval around 0 of length at least $2 \pi b(\psi(z)-\psi(y))$. This means that we can choose $y_{1} \in B_{\Delta /|b|}\left(y_{0}\right)$ such that

$$
b\left(\psi\left(y_{1}\right)-\psi(y)\right)=\theta\left(y_{0}\right)-\pi \bmod 2 \pi .
$$

Note that $\theta\left(y_{0}\right)-V\left(y_{0}\right)+b \psi\left(y_{0}\right)=0$. Using the above displayed equation,

$$
\theta\left(y_{1}\right)-\pi=V\left(y_{1}\right)-b \psi\left(y_{1}\right)-\pi+\theta\left(y_{0}\right)-V\left(y_{0}\right)+b \psi\left(y_{0}\right)=V\left(y_{1}\right)-V\left(y_{0}\right) .
$$

Together with (6.6), the above equation implies that $\left|\theta\left(y_{1}\right)-\pi\right| \leq \pi / 6$. Recalling $\sup _{Y}\left|\psi^{\prime}\right| \leq C_{0}$ and our choice of $\delta$,

$$
\begin{aligned}
\left|\sup _{B_{\delta /|b|}\left(y_{1}\right)} \theta-\pi\right| & \leq \frac{\pi}{6}+\sup _{B_{\delta /|b|}\left(y_{1}\right)}\left|\theta-\theta\left(y_{1}\right)\right| \\
& \leq \frac{\pi}{6}+|b| \sup _{B_{\delta /|b|}\left(y_{1}\right)}\left|\psi-\psi\left(y_{1}\right)\right|+\operatorname{Osc}_{B_{\delta /|b|}\left(y_{1}\right)} V+\operatorname{Osc}_{B_{\Delta /|b|} \mid} V \\
& \leq \frac{\pi}{6}+C_{0} \delta+2 \operatorname{Osc}_{B_{\xi /|b|}\left(y_{0}\right)} V \leq \frac{4 \pi}{6}=\frac{2 \pi}{3},
\end{aligned}
$$

which ends the proof.

Let $I^{p}$ be a closed interval contained in an atom of $\mathcal{P}_{k}$ such that if Lemma 6.1 holds on $B_{\delta /|b|}\left(y_{1}\right)$, we also have $B_{\delta /|b|}\left(y_{1}\right) \subset I^{p}$. Write type $\left(I^{p}\right)=h_{m}$ if we are in case $h_{m}$. Then we can find finitely many disjoint intervals $I_{j}^{p}=\left[a_{j}, b_{j+1}\right], j=0, \ldots, N-1$ (with $0=b_{0} \leq a_{0}<$ $\left.b_{1}<a_{1}<\ldots<b_{N} \leq a_{n}=1\right)$ of type $\left(I_{j}^{p}\right) \in\left\{h_{1}, h_{2}\right\}$ with $\operatorname{diam}\left(I_{j}^{p}\right) \in[\delta /|b|, 2 \delta /|b|]$ and gaps $J_{j}^{p}=\left[b_{j}, a_{j}\right], j=0, \ldots, N$ with $\operatorname{diam}\left(J_{j}^{p}\right) \in(0,2 \Delta /|b|]$.

Let $\chi: Y \rightarrow[\eta, 1]$, with $\eta \in\left[\eta_{0}, 1\right)$ be a $C^{1}$ function as constructed below (as in $[1,4]$ ):

- Let $p \in \mathcal{P}_{k}, h \in \mathcal{H}_{n}$ for $n \in \mathbb{N}$ and write $\left.h\right|_{p}: p \rightarrow h(p)$. Set $\chi \equiv 1$ on $Y \backslash\left(h_{1}(p) \cup h_{2}(p)\right)$.

- On $h_{1}(p)$ we require that $\chi\left(h_{1}(y)\right)=\eta$ for all $y$ lying in the middle third of an interval of type $h_{1}$ and that $\chi\left(h_{1}(y)\right)=1$ for all $y$ not lying in an interval of type $h_{1}$.

- On $h_{2}(p)$ we require that $\chi\left(h_{2}(y)\right)=\eta$ for all $y$ lying in the middle third of an interval of type $h_{2}$ and that $\chi\left(h_{2}(y)\right)=1$ for all $y$ not lying in an interval of type $h_{2}$.

Since $\operatorname{diam}\left(I_{j}^{p}\right) \geq \delta /|b|$, we can choose $\chi$ to be $C^{1}$ with $\left|\chi^{\prime}\right| \leq \frac{3(1-\eta)|b|}{\delta P}$ where $P=$ $\min _{m=1,2}\left\{\inf \left|h_{m}^{\prime}\right|\right\}$. From here on we choose $\eta \in\left[\eta_{0}, 1\right)$ sufficiently close to 1 so that $\left|\chi^{\prime}\right| \leq|b|$.

Since $p \in \mathcal{P}_{k}$ is arbitrary in the statement of Lemma 6.1 and the construction of $\chi$ above, we obtain

Corollary 6.2. Let $\delta, \Delta$ be as in Lemma 6.1. Let $|b| \geq 4 \pi / D$ and $(u, v) \in \mathcal{C}_{b}$. Let $\chi=\chi(b, u, v)$ be the $C^{1}$ function described above. Then $\left|\tilde{\mathcal{L}}_{s}^{n_{0}} v(y)\right| \leq \tilde{\mathcal{L}}_{\sigma}^{n_{0}}(\chi u)(y)$, for all $s=\sigma+i b,|\sigma|<\varepsilon$ and all $y \in Y$.

The following intervals $\hat{I}^{p}$ and $\hat{J}^{p}$ are constructed as in [1, 4]. Let $\hat{I}^{p}=\cup_{j=0}^{N-1} \hat{I}_{j}^{p}$, where $\hat{I}_{j}^{p}$ denotes the middle third of $I_{j}^{p}$. Let $\hat{J}_{j}$ be the interval consisting of $J_{j}$ together with the rightmost third of $I_{j-1}^{p}$ and the leftmost third of $I_{j}^{p}$. Define $\hat{J}_{0}^{p}$ and $\hat{J}_{p}^{N}$ with the obvious modifications. By construction, $\operatorname{diam}\left(\hat{I}_{j}^{p}\right) \geq \frac{1}{3} \frac{\delta}{|b|}$ and $\operatorname{diam}\left(\hat{J}_{j}^{p}\right) \geq\left(\frac{4}{3}+2 \Delta\right) \frac{\delta}{|b|}$. Hence, there is a constant $\delta^{\prime}=\delta /(4 \delta+6 \Delta)>0$ (independent of $b$ ) such that $\operatorname{diam}\left(\hat{I}_{j}^{p}\right) \geq \delta^{\prime} \operatorname{diam}\left(\hat{J}_{j}^{p}\right)$ for $j=0, \ldots, N-1$.

Proposition 6.3. Suppose that $w$ is a positive function with $\frac{\sup _{p} w}{\inf _{p} w} \leq M$ for some $M>0$. Then $\int_{\hat{I}^{p}} w d L e b \geq \delta^{\prime \prime} \int_{\hat{J}^{p}} w d L e b$, where $\delta^{\prime \prime}=(2 M)^{-1} \delta^{\prime}$.

Proof. Compute that

$$
\begin{aligned}
\int_{\hat{I}^{p}} w d \operatorname{Leb} & \geq \operatorname{Leb}\left(\hat{I}_{j}^{p}\right) \inf _{p} w \geq M^{-1} \delta^{\prime} \operatorname{Leb}\left(\hat{J}_{j}^{p}\right) \sup _{p} w \\
& =2 \delta^{\prime \prime} \operatorname{Leb}\left(\hat{J}_{j}^{p}\right) \inf _{p} w \geq 2 \delta^{\prime \prime} \int_{\hat{J}_{j}^{p}} w d \operatorname{Leb} .
\end{aligned}
$$


Here the factor 2 takes care of the intervals $\hat{J}_{0}^{p}$ and $\hat{J}_{p}^{N}$.

\section{Invariance of the cone}

Recall that the cone $\mathcal{C}_{b}$ was defined in (6.1). The main result of this section is:

Lemma 7.1. Assume $|b| \geq 2$. Then $\mathcal{C}_{b}$ is invariant under $(u, v) \mapsto\left(\tilde{\mathcal{L}}_{\sigma}^{n_{0}}(\chi u), \tilde{\mathcal{L}}_{s}^{n_{0}} v\right)$, where $\chi=\chi(b, u, v) \in C^{1}(Y)$ comes from Corollary 6.2.

Proof. Since $\chi u \geq \eta u>0$ and $\tilde{\mathcal{L}}_{\sigma}$ is a positive operator we have $\tilde{\mathcal{L}}_{\sigma}^{n_{0}}(\chi u)>0$. The condition $\left|\tilde{\mathcal{L}}_{s}^{n_{0}} v\right| \leq \tilde{\mathcal{L}}_{\sigma}^{n_{0}}(\chi u)$ follows from Corollary 6.2. In what follows we check the other cone conditions for the pair $\left(\tilde{\mathcal{L}}_{\sigma}^{n_{0}}(\chi u), \tilde{\mathcal{L}}_{s}^{n_{0}} v\right)$.

For simplicity of exposition, we assume that $n_{0}=2 q k$ for some $q \geq 1$. We will start with invariance of the exponential jump-size and oscillation conditions under $(u, v) \mapsto\left(\tilde{\mathcal{L}}_{\sigma}^{n} u, \tilde{\mathcal{L}}_{s}^{n} v\right)$ for a smaller exponent $n=2 k$. Iterating this, we get to the required exponent $n_{0}$. Hence define

$$
\begin{array}{rcc}
\left(u_{1}, v_{1}\right) & =\left(\tilde{\mathcal{L}}_{\sigma}^{n} u, \tilde{\mathcal{L}}_{s}^{n} v\right) \\
\left(u_{2}, v_{2}\right) & = & \left(\tilde{\mathcal{L}}_{\sigma}^{n} u_{1}, \tilde{\mathcal{L}}_{s}^{n} v_{1}\right) \\
\vdots & \vdots & \vdots \\
\left(u_{q-1}, v_{q-1}\right) & = & \left(\tilde{\mathcal{L}}_{\sigma}^{n} u_{q-2}, \tilde{\mathcal{L}}_{s}^{n} v_{q-2}\right) \\
\left(u_{q}, v_{q}\right) & = & \left(\tilde{\mathcal{L}}_{\sigma}^{n} u_{q-1}, \tilde{\mathcal{L}}_{s}^{n} v_{q-1}\right)=\left(\tilde{\mathcal{L}}_{\sigma}^{n_{0}} u, \tilde{\mathcal{L}}_{s}^{n_{0}} v\right) .
\end{array}
$$

Since $|v| \leq u$, this construction shows that $|v| \leq u$ for all $1 \leq i \leq q$. We will now show by induction that $\left(u_{i}, v_{i}\right)$ satisfies (4.4) and $\operatorname{Osc}_{I} v_{i} \leq C_{10}|b| \operatorname{Leb}(I) \sup _{I} u_{i}+C_{8} E_{I}\left(u_{i}\right)$ for all $1 \leq i \leq q$.

The 'exponential decrease of jump-sizes' condition in $\mathcal{C}_{b}$. Without loss of generality we can refine (if needed) the partition $\mathcal{P}_{k}$ such that

$$
C_{10}|b| \operatorname{Leb}\left(\left[\xi_{i-1}, \xi_{i}\right]\right) \leq \frac{2}{3},
$$

for all $i$. Then the oscillation condition applied to $(u, v=u)$ combined with (7.1) and the fact that $E_{I}(u) \leq \frac{1}{12} \sup _{p} u$ give $\sup _{p} u-\inf _{p} u=\operatorname{Osc}_{p} u \leq\left(\frac{2}{3}+\frac{1}{12}\right) \sup _{p} u$. Therefore $\frac{\left.\sup u\right|_{p}}{\left.\inf u\right|_{p}} \leq 4$ for each $p \in \mathcal{P}_{k}$. The invariance of the exponential jump-size condition follows by Proposition 5.3, that is: the pair $\left(\tilde{\mathcal{L}}_{\sigma}^{n} u, \tilde{\mathcal{L}}_{s}^{n} v\right)$ satisfies (4.4) as well.

The 'oscillation' condition in $\mathcal{C}_{b}$. For the invariance of the oscillation condition, we need to verify

$$
\operatorname{Osc}_{I}\left(\tilde{\mathcal{L}}_{s}^{n} v\right) \leq C_{10}|b| \operatorname{Leb}(I) \sup _{x \in I}\left(\tilde{\mathcal{L}}_{\sigma}^{n} u\right)(x)+C_{8} E_{I}\left(\tilde{\mathcal{L}}_{\sigma}^{n} u\right) .
$$

For this purpose, we $\operatorname{split}^{\operatorname{Osc}_{I}}\left(\tilde{\mathcal{L}}_{s}^{n} v\right)$ into a sum of jump-sizes at non-onto branches (i.e., $\partial \operatorname{dom}(h) \cap$ $I^{\circ} \neq \emptyset$, corresponding to the "created" discontinuities), and a sum of onto branches (which includes "propagated" discontinuities). Because of (4.2), this gives the following:

$$
\begin{aligned}
\operatorname{Osc}_{I}\left(\tilde{\mathcal{L}}_{s}^{n} v\right) \leq & \sum_{h \in \mathcal{H}_{n}, \partial \operatorname{dom}(h) \cap I^{\circ} \neq \emptyset} \operatorname{Size}\left(\left|h^{\prime}\right| e^{s \varphi_{n} \circ h(x)} \frac{\left(f_{\sigma} v\right) \circ h}{\lambda_{\sigma}^{n} f_{\sigma}}\right)\left(\partial \operatorname{dom}(h) \cap I^{\circ}\right) \\
& +\sum_{h \in \mathcal{H}_{n}, \operatorname{dom}(h) \cap I^{\circ} \neq \emptyset} \operatorname{Osc}_{I}\left(\left|h^{\prime}\right| e^{s \varphi_{n} \circ h} \frac{\left(f_{\sigma} v\right) \circ h}{\lambda_{\sigma}^{n} f_{\sigma}}\right) \\
= & O_{1}+O_{2} .
\end{aligned}
$$

For the term $O_{1}$ we use Proposition 5.3, and recall that $I \subset p$, so each created discontinuity $x$ in this sum belong to $X_{j}^{\prime}$ for some $k<j \leq n$. We obtain

$$
O_{1} \leq C_{7} \sum_{j=k+1}^{n} \rho^{-j} \sum_{x \in X_{j}^{\prime} \cap I^{\circ}} \tilde{\mathcal{L}}_{\sigma}^{n} u(x),
$$


which contributes to $E_{I}\left(\tilde{\mathcal{L}}_{\sigma}^{n}(\chi u)\right)$.

Now for the sum $\mathrm{O}_{2}$ (concerning the interiors of $\operatorname{dom}(h), h \in \mathcal{H}_{n}$ ), we decompose the summands into five parts, according to the five factors $\left|h^{\prime}\right|, e^{s \varphi_{n} \circ h}, f_{\sigma} \circ h, 1 / f_{\sigma}$ and $v \circ h$ of which the oscillations have to be estimated. The estimates for this five parts are as follows.

The term with $\left|h^{\prime}\right|$. For each $h \in \mathcal{H}_{n}$ we have $1=h^{\prime} \circ F^{n} \cdot\left(F^{n}\right)^{\prime}$ and $0=h^{\prime \prime} \circ F^{n} \cdot\left(\left(F^{n}\right)^{\prime}\right)^{2}+$ $h^{\prime} \circ F^{n} \cdot\left(F^{n}\right)^{\prime \prime}$. Using Adler's condition (2.2) for the branches of $F^{n}$,

$$
\left|h^{\prime \prime}(\xi)\right|=\frac{\left|\left(F^{n}\right)^{\prime \prime} \circ h(\xi)\right|}{\left|\left(F^{n}\right)^{\prime} \circ h(\xi)\right|^{2}} \cdot\left|h^{\prime}(\xi)\right| \leq C_{1}\left|h^{\prime}(\xi)\right|
$$

for each $n \geq 1$ and $\xi \in a \in \alpha^{n}$. Hence by the Mean Value Theorem,

$$
\operatorname{Osc}_{I^{\circ}}\left(\left|h^{\prime}\right|\right) \leq \operatorname{Leb}(I)\left|h^{\prime \prime}(\xi)\right| \leq C_{1} \operatorname{Leb}(I)\left|h^{\prime}(\xi)\right| \leq C_{1} e^{C_{1}} \operatorname{Leb}(I) \inf _{x \in \operatorname{dom}(h) \cap I}\left|h^{\prime}(x)\right| .
$$

Summing over all $h \in \mathcal{H}_{n}$ with $\operatorname{dom}(h) \cap I^{\circ} \neq \emptyset$, we get

$$
\sum_{\substack{h \in \mathcal{H}_{n} \\ \operatorname{dom}(h) \cap I^{\circ} \neq \emptyset}} \operatorname{Osc}_{I^{\circ}}\left(\left|h^{\prime}\right|\right) \sup _{x \in \operatorname{dom}(h) \cap I^{\circ}} e^{\sigma \varphi_{n} \circ h(x)} \frac{\left(f_{\sigma}|v|\right) \circ h(x)}{\lambda_{\sigma}^{n} f_{\sigma}(x)} \leq C_{1} e^{C_{1}} \operatorname{Leb}(I) \sup _{x \in I}\left(\tilde{\mathcal{L}}_{\sigma}^{n} u\right)(x) .
$$

The term with $e^{s \varphi_{n} \circ h}$. Write $\varphi_{n}(x)=\sum_{i=0}^{m-1} \varphi \circ F^{i}(x)$ and $h=h_{n} \circ h_{n-1} \circ \cdots \circ h_{1} \in \mathcal{H}_{n}$ where $h_{j} \in \mathcal{H}_{1}$ for $1 \leq j \leq n$. Then by $(2.3)$

$$
\begin{aligned}
\left|\left(\varphi_{n} \circ h\right)^{\prime}\right| & \leq \sum_{j=0}^{n-1}\left|\left(\varphi \circ h_{n-j} \circ F^{j+1} \circ h\right)^{\prime}\right|=\sum_{j=0}^{n-1}\left|\left(\varphi \circ h_{n-j}\right)^{\prime}\right| \cdot\left|\left(F^{j+1} \circ h\right)^{\prime}\right| \\
& \leq C_{2} \sum_{j=0}^{n-1} \rho_{0}^{-(n-(j+1))} \leq \frac{C_{2} \rho_{0}}{\rho_{0}-1}=: C_{2}^{\prime} .
\end{aligned}
$$

By the Mean Value Theorem $\frac{\sup _{x \in I} e^{\sigma \varphi_{n} \circ h(x)}}{\inf _{x \in I} e^{\sigma \varphi_{n} \circ h(x)}} \leq e^{\sigma\left(\varphi_{n} \circ h\right)^{\prime}(\xi) \operatorname{Leb}(I)} \leq e^{\varepsilon C_{2}^{\prime}}$. Therefore

$$
\begin{aligned}
\operatorname{Osc}_{I^{\circ}}\left(e^{s \varphi_{n} \circ h}\right) & =|s| e^{\sigma \varphi_{n} \circ h(\xi)}\left|\left(\varphi_{n} \circ h\right)^{\prime}(\xi)\right| \operatorname{Leb}(I) \\
& \leq(1+\varepsilon)|b| \frac{\sup _{x \in I} e^{\sigma \varphi_{n} \circ h(x)}}{\inf _{x \in I} e^{\sigma \varphi_{n} \circ h(x)}} \inf _{x \in I} e^{\sigma \varphi_{n} \circ h(x)} \sup _{x \in I}\left(\varphi_{n} \circ h\right)^{\prime}(x) \\
& \leq(1+\varepsilon) e^{\varepsilon C_{2}^{\prime}} C_{2}^{\prime}|b| \operatorname{Leb}(I) \inf _{x \in I} e^{\sigma \varphi_{n} \circ h(x)} .
\end{aligned}
$$

Summing over all $h \in \mathcal{H}_{n}$ with $\operatorname{dom}(h) \cap I^{\circ} \neq \emptyset$, this gives

$$
\begin{aligned}
& \sum_{\substack{h \in \mathcal{H}_{n} \\
\operatorname{dom}(h) \cap I^{\circ} \neq \emptyset}} \operatorname{Osc}_{I^{\circ}}\left(e^{s \varphi_{n} \circ h}\right) \sup _{x \in \operatorname{dom}(h) \cap I^{\circ}}\left|h^{\prime}(x)\right| \frac{\left(f_{\sigma}|v|\right) \circ h(x)}{\lambda_{\sigma}^{n} f_{\sigma}(x)} \\
& \leq(1+\varepsilon) e^{\varepsilon C_{2}^{\prime}} C_{2}^{\prime}|b| \operatorname{Leb}(I) \sup _{x \in I}\left(\tilde{\mathcal{L}}_{\sigma}^{n} u\right)(x) .
\end{aligned}
$$

The term with $f_{\sigma} \circ h$. Applying Lemma 5.1, part 2 to $f_{\sigma} \circ h$ we find

$$
\operatorname{Osc}_{I^{\circ}}\left(f_{\sigma} \circ h\right) \leq C_{6} \operatorname{Leb}(h(I)) \inf _{x \in h(I)} f_{\sigma}(x)+C_{7} E_{h(I)}\left(f_{\sigma}\right) .
$$

For an arbitrary $h \in \mathcal{H}_{n}$, the first term in (7.7), multiplied by $\sup _{x \in \operatorname{dom}(h) \cap I^{\circ}}\left|h^{\prime}(x)\right|\left|e^{s \varphi_{n} \circ h(x)}\right| \frac{|v| \circ h(x)}{\lambda_{\sigma}^{n} f_{\sigma}(x)}$ is bounded by

$$
C_{6} \operatorname{Leb}(h(I)) \sup _{x \in \operatorname{dom}(h) \cap I^{\circ}}\left|h^{\prime}(x)\right| e^{\sigma \varphi_{n} \circ h(x)} \frac{\left(f_{\sigma} u\right) \circ h(x)}{\lambda_{\sigma}^{n} f_{\sigma}(x)} .
$$


Summing over all $h \in \mathcal{H}_{n}$ with $\operatorname{dom}(h) \cap I^{\circ} \neq \emptyset$ gives

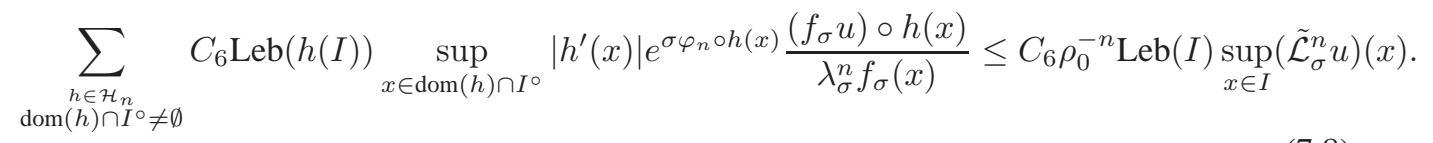

The second term in (7.7) is a sum over propagated discontinuities $x \in I^{\circ}$, and for each $x$ we let $\tilde{h} \in \mathcal{H}_{n}$ be the inverse branch such that $f_{\sigma}$ has a discontinuity at $y=\tilde{h}(x)$, and $j>k$ is such that $x \in X_{j}^{\prime}$. By Lemma 5.1 the term in $E_{h(I)}\left(f_{\sigma}\right)$ related to $y$ is bounded by $C_{7} \rho^{-3(j-n)} f_{\sigma}(y)$. Multiplied by $\left|\tilde{h}^{\prime}(x)\right|\left|e^{s \varphi_{n} \circ \tilde{h}(x)}\right| \frac{|v| \circ \tilde{h}(x)}{\lambda_{\sigma}^{n} f_{\sigma}(x)}$, and using (5.2) to obtain an upper bound for $u \circ \tilde{h}(x)=$ $u(y)$, this gives

$$
\begin{aligned}
\frac{C_{7}}{\rho^{3(j-n)}} f_{\sigma}(y)\left|\tilde{h}^{\prime}(x)\right| e^{\sigma \varphi_{n} \circ \tilde{h}(x)} \frac{|v| \circ \tilde{h}(x)}{\lambda_{\sigma}^{n} f_{\sigma}(x)} & \leq \frac{C_{7}}{\rho^{3(j-n)}} \rho^{-3 n} \frac{\left(f_{\sigma} u\right) \circ \tilde{h}(x)}{\lambda_{\sigma}^{n} f_{\sigma}(x)} \\
& \leq C_{7} \rho^{-j} \frac{\sup f_{\sigma}}{\inf f_{\sigma}} \frac{\left.\sup u\right|_{p}}{\left.\inf u\right|_{p}} \frac{1}{\rho^{k} C_{9} \operatorname{Leb}(p)} \tilde{\mathcal{L}}_{\sigma}^{n} u(x) .
\end{aligned}
$$

Since $\frac{\left.\sup u\right|_{p}}{\left.\inf u\right|_{p}} \leq 4$, the bound on $\operatorname{Leb}(p)$ in (2.5) gives $\frac{\sup f_{\sigma}}{\inf f_{\sigma}} \frac{\left.\sup u\right|_{p}}{\left.\inf u\right|_{p}} \frac{1}{\rho^{k} C_{9} \operatorname{Leb}(p)} \leq 1$. Hence, summing over all propagated discontinuities $x \in I^{\circ}$ and corresponding branches, we get

$$
C_{7} \sum_{j>n} \sum_{x \in X_{j}^{\prime} \cap I^{\circ}} \rho^{-3(j-n)} f_{\sigma}(y)\left|h^{\prime}(x)\right| e^{\sigma \varphi_{n} \circ h(x)} \frac{|v| \circ h(x)}{\lambda_{\sigma}^{n} f_{\sigma}(x)} \leq C_{7} \sum_{j>n} \rho^{-j} \sum_{x \in X_{j}^{\prime} \cap I^{\circ}} \tilde{\mathcal{L}}_{\sigma}^{n} u(x) .
$$

which contributes to $E_{I}\left(\tilde{\mathcal{L}}_{\sigma}^{n} u\right)$.

The term with $1 / f_{\sigma}$. Applying Lemma 5.1, part 2. to $f_{\sigma} \circ h$ we find

$$
\operatorname{Osc}_{I^{\circ}}\left(1 / f_{\sigma}\right) \leq C_{6} \operatorname{Leb}(I) \inf _{x \in h(I)} 1 / f_{\sigma}(x)+C_{7} E_{I}\left(1 / f_{\sigma}\right) .
$$

For $h \in \mathcal{H}_{n}$, the first term of (7.10), multiplied by $\sup _{x \in \operatorname{dom}(h) \cap I^{\circ}}\left|h^{\prime}(x)\right|\left|e^{s \varphi_{n} \circ h(x)}\right| \frac{\left(f_{\sigma}|v|\right) \circ h(x)}{\lambda_{\sigma}^{n}}$ is bounded by

$$
C_{6} \operatorname{Leb}(I) \sup _{x \in \operatorname{dom}(h) \cap I^{\circ}}\left|h^{\prime}(x)\right| e^{\sigma \varphi_{n} \circ h(x)} \frac{\left(f_{\sigma} u\right) \circ h(x)}{\lambda_{\sigma}^{n} f_{\sigma}(x)} .
$$

Summing over all $h \in \mathcal{H}_{n}$ with $\operatorname{dom}(h) \cap I^{\circ} \neq \emptyset$ gives

$$
\sum_{\substack{h \in \mathcal{H}_{n} \\ \operatorname{dom}(h) \cap I^{\circ} \neq \emptyset}} C_{6} \operatorname{Leb}(I) \sup _{x \in \operatorname{dom}(h) \cap I^{\circ}}\left|h^{\prime}(x)\right| e^{\sigma \varphi_{n} \circ h(x)} \frac{\left(f_{\sigma} u\right) \circ h(x)}{\lambda_{\sigma}^{n} f_{\sigma}(x)} \leq C_{6} \operatorname{Leb}(I) \sup _{x \in I}\left(\tilde{\mathcal{L}}_{\sigma}^{n} u\right)(x) .
$$

The second term of (7.10) is a sum over propagated discontinuities $x \in I^{\circ}$. Take $j>k$ such that $x \in X_{j}^{\prime}$. Lemma 5.1 gives that the term in $E_{I}$ related to $x$ is bounded by $C_{7} \rho^{-3 j} / f_{\sigma}(x)$. Multiplying with $\left|h^{\prime}(x)\right|\left|e^{\sigma \varphi_{n} \circ h(x)}\right| \frac{\left(f_{\sigma} u\right) \circ h(x)}{\lambda_{\sigma}^{n}}$ and then summing over all $x \in \cup_{j>k} X_{j}^{\prime} \cap I^{\circ}$ and $h \in \mathcal{H}_{n}$ with $x \in \operatorname{dom}(h)$ gives

$$
C_{7} \sum_{j>k} \rho^{-3 j} \sum_{x \in X_{j}^{\prime} \cap I^{\circ}}\left|h^{\prime}(x)\right| e^{\sigma \varphi_{n} \circ h(x)} \frac{\left(f_{\sigma} u\right) \circ h(x)}{\lambda_{\sigma}^{n} f_{\sigma}(x)} \leq C_{7} \sum_{j>k} \rho^{-j} \sum_{x \in X_{j}^{\prime} \cap I^{\circ}}\left(\tilde{\mathcal{L}}_{\sigma}^{n} u\right)(x),
$$

which contributes to $E_{I}\left(\tilde{\mathcal{L}}_{\sigma}^{n} u\right)$.

The term with $v$. Using the cone condition for $v$, we obtain

$$
\begin{aligned}
\operatorname{Osc}_{I^{\circ}}(v \circ h) & \leq C_{10} \operatorname{Leb}(h(I))|b| \sup _{x \in h(I)} u(x)+C_{8} E_{h(I)}(u) \\
& \leq \rho_{0}^{-n} \frac{\left.\sup u\right|_{h(I)}}{\left.\inf u\right|_{h(I)}} C_{10} \operatorname{Leb}(I)|b| \inf _{x \in h(I)} u(x)+C_{8} E_{h(I)}(u) .
\end{aligned}
$$


For $h \in \mathcal{H}_{n}$, the first term of (7.13), multiplied by $\sup _{x \in \operatorname{dom}(h) \cap I^{\circ}}\left|h^{\prime}(x)\right|\left|e^{s \varphi_{n} \circ h(x)}\right| \frac{f_{\sigma} \circ h(x)}{\lambda_{\sigma}^{n} f_{\sigma}(x)}$, is bounded by

$$
4 \rho_{0}^{-n} C_{10}|b| \operatorname{Leb}(I) \sup _{x \in \operatorname{dom}(h) \cap I^{\circ}}\left|h^{\prime}(x)\right| e^{\sigma \varphi_{n} \circ h(x)} \frac{\left(f_{\sigma} u\right) \circ h(x)}{\lambda_{\sigma}^{n} f_{\sigma}(x)} .
$$

Summing over all $h \in \mathcal{H}_{n}$ with $\operatorname{dom}(h) \cap I^{\circ} \neq \emptyset$ gives

$$
\sum_{\substack{h \in \mathcal{H}_{n} \\ \operatorname{dom}(h) \cap I^{\circ} \neq \emptyset}} \frac{4 C_{10}}{\rho_{0}^{n}}|b| \operatorname{Leb}(I) \sup _{x \in \operatorname{dom}(h) \cap I^{\circ}}\left|h^{\prime}(x)\right| e^{\sigma \varphi_{n} \circ h(x)} \frac{\left(f_{\sigma} u\right) \circ h(x)}{\lambda_{\sigma}^{n} f_{\sigma}(x)} \leq \frac{4 C_{10}}{\rho_{0}^{n}}|b| \operatorname{Leb}(I) \sup _{x \in I}\left(\tilde{\mathcal{L}}_{\sigma}^{n} u\right)(x) .
$$

The second term of (7.13) is a sum over propagated discontinuities $x \in I^{\circ}$. For each such $x$ we let $\tilde{h} \in \mathcal{H}_{n}$ be the inverse branch such that $v$ has a discontinuity at $y=\tilde{h}(x)$, and $j$ is such that $x \in X_{j}^{\prime}$.

Case a: Assume that $j-n>k$. Since $u$ has exponentially decreasing jump-sizes, we get that the term in $E_{h(I)}$ related to $y$ is bounded by $C_{7} \rho^{-(j-n)} u(y)$. After multiplying by $\left|\tilde{h}^{\prime}(x)\right|\left|e^{s \varphi_{n} \circ \tilde{h}(x)}\right| \frac{f_{\sigma} \circ \tilde{h}(x)}{\lambda_{\sigma}^{n} f_{\sigma}(x)}$, and using (5.2) for an upper bound of $u \circ \tilde{h}(x)=u(y)$, we have

$$
\begin{aligned}
C_{7} \rho^{-(j-n)} u(y)\left|h^{\prime}(x)\right| e^{\sigma \varphi_{n} \circ h(x)} \frac{f_{\sigma} \circ h(x)}{\lambda_{\sigma}^{n} f_{\sigma}(x)} & \leq C_{7} \rho^{-(j-n)} \rho^{-3 n} \frac{\left(f_{\sigma} u\right) \circ \tilde{h}(x)}{f_{\sigma}(x)} \\
& \leq C_{7} \rho^{-j} \frac{\sup f_{\sigma}}{\inf f_{\sigma}} \frac{\left.\sup u\right|_{p}}{\left.\inf u\right|_{p}} \frac{1}{\rho^{k} C_{9} \operatorname{Leb}(p)} \tilde{\mathcal{L}}_{\sigma}^{n} u(x) \\
& \leq \frac{C_{7}}{C_{8}} \rho^{-j} \tilde{\mathcal{L}}_{\sigma}^{n} u(x),
\end{aligned}
$$

because $\frac{\left.\sup u\right|_{p}}{\left.\inf u\right|_{p}} \leq 4$, and using the bound on $\operatorname{Leb}(p)$ from (2.5).

Case b: Assume that $j-n \leq k$. Then (4.1) doesn't apply to the term in $E_{h(I)}$ related to $y$, so it can only be bounded by $u(y)$. Multiplied by $\left|\tilde{h}^{\prime}(x)\right|\left|e^{s \varphi_{n} \circ \tilde{h}(x)}\right| \frac{f_{\sigma} \circ \tilde{h}(x)}{\lambda_{\sigma}^{n} f_{\sigma}(x)}$, and using (5.2) for obtaining an upper bound of $u \circ \tilde{h}(x)=u(y)$, we have

$$
\begin{aligned}
u(y)\left|h^{\prime}(x)\right| e^{\sigma \varphi_{n} \circ h(x)} \frac{f_{\sigma} \circ h(x)}{\lambda_{\sigma}^{n} f_{\sigma}(x)} & \leq \rho^{-3 n} \frac{\left(f_{\sigma} u\right) \circ \tilde{h}(x)}{f_{\sigma}(x)} \\
& \leq \rho^{-2(n-k)} \frac{\sup f_{\sigma}}{\inf f_{\sigma}} \frac{\left.\sup u\right|_{p}}{\left.\inf u\right|_{p}} \frac{1}{\rho^{k} C_{9} \operatorname{Leb}(p)} \rho^{-j} \tilde{\mathcal{L}}_{\sigma}^{n} u(x) \\
& \leq \frac{1}{C_{8}} \rho^{-j} \tilde{\mathcal{L}}_{\sigma}^{n} u(x),
\end{aligned}
$$

because $\frac{\left.\sup u\right|_{p}}{\left.\inf u\right|_{p}} \leq 4$, and using the bound on $\operatorname{Leb}(p)$ from (2.5). Hence, summing over all propagated discontinuities $x \in I^{\circ}$ and corresponding branches, we get

$$
C_{7} \sum_{j>n} \sum_{x \in X_{j}^{\prime} \cap I^{\circ}} \rho^{-(j-n)} f_{\sigma}(y)\left|h^{\prime}(x)\right| e^{\sigma \varphi_{n} \circ h(x)} \frac{|v| \circ h(x)}{\lambda_{\sigma}^{n} f_{\sigma}(x)} \leq \frac{C_{7}}{C_{8}} \sum_{j>n} \rho^{-j} \sum_{x \in X_{j}^{\prime} \cap I^{\circ}} \tilde{\mathcal{L}}_{\sigma}^{n} u(x),
$$

which contributes to $E_{I}\left(\tilde{\mathcal{L}}_{\sigma}^{n} u\right)$. This completes the treatment of the five terms.

Combining terms (7.4), (7.6), (7.8), (7.11) and (7.14), the oscillation part is bounded by

$$
\left(C_{1} e^{C_{1}}+(1+\varepsilon)|b| e^{\varepsilon C_{2}^{\prime}} C_{2}^{\prime}+\left(1+\rho_{0}^{-n}\right) C_{6}+4 C_{10} \rho_{0}^{-n}\right) \operatorname{Leb}(I) \sup _{I}\left(\tilde{\mathcal{L}}_{\sigma}^{n} u\right)
$$

and by the choice of $C_{10}$ in Subsection 2.7, this is less than $C_{10}|b| \operatorname{Leb}(I) \eta_{0} \sup _{I}\left(\mathcal{L}_{\sigma}^{n} u\right)$ whenever $|b| \geq 2$.

Recall $C_{8}=3 C_{7} / \eta_{0}$. Combining (7.2), (7.9), (7.12) and (7.15), the jump part is bounded by

$$
3 C_{7} E_{I}\left(\tilde{\mathcal{L}}^{n} u\right) \leq C_{8} \eta_{0} E_{I}\left(\tilde{\mathcal{L}}^{n} u\right) .
$$


This concludes the induction step, proving that

$$
\begin{aligned}
\operatorname{Osc}_{I^{\circ}}\left(\tilde{\mathcal{L}}_{s}^{n_{0}} v\right) & \leq C_{10} \eta_{0}|b| \operatorname{Leb}(I) \sup _{I}\left(\tilde{\mathcal{L}}_{\sigma}^{n_{0}} u\right)+C_{8} \eta_{0} E_{I}\left(\tilde{\mathcal{L}}_{\sigma}^{n_{0}} u\right) \\
& \leq C_{10}|b| \operatorname{Leb}(I) \sup _{I}\left(\tilde{\mathcal{L}}_{\sigma}^{n_{0}}(\chi u)\right)+C_{8} E_{I}\left(\tilde{\mathcal{L}}_{\sigma}^{n_{0}}(\chi u)\right)
\end{aligned}
$$

as required.

\section{Proof of Theorem 2.3}

Given Lemma 6.1 and Lemma 7.1, the proof of the $L^{2}$ contraction for functions in $\mathcal{C}_{b}$ goes almost word by word as the proof of [1, Theorem 2.16] with some obvious modifications. We sketch the argument in Subsection 8.1. In Subsection 8.2 we deal with arbitrary BV observables satisfying a mild condition via the \|\|$_{b}$ norm. In Subsection 8.3, we complete the argument required for the proof of Theorem 2.3.

\section{1 $L^{2}$ contraction for functions in $\mathcal{C}_{b}$}

Lemma 8.1. There exist $\varepsilon \in(0,1)$ and $\beta \in(0,1)$ such that for all $m \geq 1, s=\sigma+i b,|\sigma|<\varepsilon$, $|b| \geq \max \{4 \pi / D, 2\}$,

$$
\int\left|\tilde{\mathcal{L}}_{s}^{m n_{0}} v\right|^{2} d L e b \leq \beta^{m}\|v\|_{\infty}^{2}
$$

for all $v \in B V$ such that $(u, v)$ for $u=$ cst satisfy condition (4.4) in Definition 4.1.

Proof. Set $u_{0} \equiv\|v\|_{\infty}, v_{0}=v$ and for $m \geq 0$, define

$$
u_{m+1}=\tilde{\mathcal{L}}_{\sigma}^{n_{0}}\left(\chi_{m} u_{m}\right), \quad v_{m+1}=\tilde{\mathcal{L}}_{s}\left(v_{m}\right),
$$

where $\chi_{m}$ is a function depending on $b, u_{m}, v_{m}$. Since by definition $\left(u_{0}, v_{0}\right) \in \mathcal{C}_{b}$, it follows from Lemma 7.1 that $\left(u_{m}, v_{m}\right) \in \mathcal{C}_{b}$, for all $m$. Thus, we can construct $\chi_{m}:=\chi\left(b, u_{m}, v_{m}\right)$ inductively as in Corollary 6.2.

As in [1, 4], it is enough to show that there exists $\beta \in(0,1)$ such that $\int u_{m+1}^{2} d$ Leb $\leq$ $\beta \int u_{m}^{2} d$ Leb for all $m \geq 0$. Then $\left|\tilde{\mathcal{L}}_{s}^{m n_{0}} v\right|=\left|\tilde{\mathcal{L}}_{s}^{m n_{0}} v_{0}\right|=\left|v_{m}\right| \leq u_{m}$ and thus,

$$
\int\left|\tilde{\mathcal{L}}_{s}^{m n_{0}} v\right|^{2} d \text { Leb } \leq \int u_{m}^{2} d \text { Leb } \leq \beta^{m} \int u_{0}^{2} d \text { Leb }=\beta^{m}\|v\|_{\infty}^{2},
$$

as required.

Let $\hat{I}^{p}, \hat{J}^{p}$ be as constructed before the statement of Proposition 6.3 and note that $Y=$ $\left(\cup_{p} \hat{I}^{p}\right) \cup\left(\cup_{p} \hat{J}^{p}\right)$. Proceeding as in the proof of [1, Lemma 2.13] (which relies on the use of the Cauchy-Schwartz inequality), we obtain that there exists $\eta_{1}<1$ such that for any $p \in \mathcal{P}_{k}$,

$$
u_{m+1}^{2}(y) \leq \begin{cases}\xi(\sigma) \eta_{1}\left(\tilde{\mathcal{L}}_{0}^{n_{0}} u_{m}^{2}\right)(y) & \text { if } y \in \hat{I}^{p} \\ \xi(\sigma)\left(\tilde{\mathcal{L}}_{0}^{n_{0}} u_{m}^{2}\right)(y) & \text { if } y \in \hat{J}^{p}\end{cases}
$$

where $\xi(\sigma)=\lambda_{\sigma}^{-2 n_{0}} \sup _{p}\left(f_{0} / f_{\sigma}\right) \sup _{p}\left(f_{2 \sigma} / f_{\sigma}\right) \sup _{p}\left(f_{\sigma} / f_{0}\right) \sup _{p}\left(f_{\sigma} / f_{2 \sigma}\right)$.

Since $\left(u_{m}, v_{m}\right) \in \mathcal{C}_{b}$, we have, in particular, that for any $p \in \mathcal{P}_{k}, \sup _{p} u_{m}-\inf _{p} u_{m} \leq$ $\operatorname{Osc}_{p} u \leq\left(\frac{2}{3}+\frac{1}{12}\right) \sup _{p} u_{m}$ and thus, $\frac{\sup _{p} u_{m}}{\inf _{p} u_{m}} \leq 4$. Similarly, $\frac{\sup _{p} u_{m}^{2}}{\inf _{p} u_{m}^{2}} \leq 16$. Hence,

$$
\begin{aligned}
\frac{\sup _{p} \tilde{\mathcal{L}}_{0}^{n_{0}}\left(u_{m}^{2}\right)}{\inf _{p} \tilde{\mathcal{L}}_{0}^{n_{0}}\left(u_{m}^{2}\right)} & =\frac{\sup _{p} \sum_{h \in \mathcal{H}_{n_{0}}}\left|h^{\prime}\right|\left(f_{0} \circ h\right)\left(u_{m}^{2} \circ h\right) / f_{0}}{\inf _{p} \sum_{h \in \mathcal{H}_{n_{0}}}\left|h^{\prime}\right|\left(f_{0} \circ h\right)\left(u_{m}^{2} \circ h\right) / f_{0}} \\
& \leq 16\left(\frac{\sup _{p} f_{0}}{\inf _{p} f_{0}}\right)^{2} \frac{\sup _{p} \sum_{h \in \mathcal{H}_{n_{0}}}\left|h^{\prime}\right|}{\inf _{p} \sum_{h \in \mathcal{H}_{n_{0}}}\left|h^{\prime}\right|}<\infty .
\end{aligned}
$$


Let $w:=\tilde{\mathcal{L}}\left(u_{m}^{2}\right)$, set $M:=16\left(\frac{\sup _{p} f_{0}}{\inf _{p} f_{0}}\right)^{2} \frac{\sup _{p} \sum_{h \in \mathcal{H}_{n}}\left|h^{\prime}\right|}{\inf _{p} \sum_{h \in \mathcal{H}_{n}}\left|h^{\prime}\right|}$ and note that $w$ satisfies the conditions of Proposition 6.3 for such $M$. For any $p \in \mathcal{P}_{k}$, it follows that $\int_{\hat{I}^{p}} w d$ Leb $\geq \delta^{\prime \prime} \int_{\hat{J}^{p}} w d$ Leb and thus,

$$
\int_{\cup_{p} \hat{I}^{p}} w d \text { Leb } \geq \delta^{\prime \prime} \int_{\cup_{p} \hat{J}^{p}} w d \text { Leb. }
$$

From here on the argument goes word by word as the argument used at the end of the proof of [1, Theorem 2.16]. We provide it here for completeness. Let $\beta^{\prime}=\frac{1+\eta_{1} \delta^{\prime \prime}}{1+\delta^{\prime \prime}}<1$. Then $\delta^{\prime \prime}=\frac{1-\beta^{\prime}}{\beta^{\prime}-\eta_{1}}$ and thus, $\left(\beta^{\prime}-\eta_{1}\right) \int_{\cup_{p} \hat{I}^{p}} w d$ Leb $\geq\left(1-\beta^{\prime}\right) \int_{\cup_{p} \hat{J}^{p}} w d$ Leb. Since also $Y=\left(\cup_{p} \hat{I}^{p}\right) \cup\left(\cup_{p} \hat{J}^{p}\right)$, we obtain $\eta_{1} \int_{\cup_{p} \hat{I}^{p}} w d$ Leb $+\int_{\cup_{p} \hat{J}^{p}} w d$ Leb $\leq \beta^{\prime} \int_{Y} w d$ Leb. Putting the above together,

$$
\begin{aligned}
\int_{Y} u_{m+1}^{2} d \text { Leb } & \leq \xi(\sigma)\left(\eta_{1} \int_{\cup_{p} \hat{I}^{p}} w d \text { Leb }+\int_{\cup_{p} \hat{J}^{p}} w d \text { Leb }\right) \\
& \leq \xi(\sigma) \beta^{\prime} \int_{Y} \tilde{\mathcal{L}}_{0}^{n_{0}}\left(u_{m+1}^{2}\right) d \text { Leb }=\xi(\sigma) \beta^{\prime} \int_{Y} u_{m}^{2} d \text { Leb. }
\end{aligned}
$$

To conclude, recall that by Remark 3.2, if necessary, we can shrink $\varepsilon$ such that $\beta:=\xi(\sigma) \beta^{\prime}<1$ for all $|\sigma|<\varepsilon$.

\subsection{Dealing with arbitrary BV observables via the \|\|$_{b}$ norm}

The cone $\mathcal{C}_{b}$ represents only a specific class of BV observables, namely with discontinuities of prescribed size and location. It is, in fact, the smallest Banach space that is invariant under $(u, v) \mapsto\left(\tilde{\mathcal{L}}_{\sigma} u, \tilde{\mathcal{L}}_{s} v\right)$ and contains all continuous BV functions.

In this section we are concerned with the behaviour of $\tilde{\mathcal{L}}_{s}^{r}$ acting on $\mathrm{BV}$ functions satisfying a certain mild condition (less restrictive than belonging to $\mathcal{C}_{b}$ ). To phrase such a condition we let $C_{11}$ be a positive constant such that

$$
C_{11}=64(1+c)^{2}\left(\frac{\sup f_{\sigma}}{\inf f_{\sigma}}\right)^{2} \frac{\sup f_{2 \sigma}}{\inf f_{2 \sigma}}\left(\frac{\sup f_{\sigma}}{\inf f_{\sigma}} \frac{\sup f_{0}}{\inf f_{0}}\right)^{2}
$$

where $c$ is the constant in the statement of Proposition 3.5. We use the following hypothesis:

$$
\left\{\begin{array}{ll}
\operatorname{Var}_{Y} v \leq C_{11}|b|^{2} \rho^{m n_{0}}\|v\|_{1} & \text { if } \sigma \geq 0, \\
\operatorname{Var}_{Y}\left(e^{\sigma \varphi_{m n_{0}}} v\right) \leq C_{11}|b|^{2} \rho^{m n_{0}}\left\|e^{\sigma \varphi_{m n_{0}}} v\right\|_{1} & \text { if } \sigma<0 .
\end{array} \quad\left(H_{\sigma, m}\right)\right.
$$

The next result, Proposition 8.2, says that for $v \in \mathrm{BV}(Y)$ such that if $\left(H_{\sigma, m}\right)$, then $\tilde{\mathcal{L}}_{s}^{r} v$ is exponentially close to the cone $\mathcal{C}_{b}$ in \|\|$_{\infty}$, because jumps-sizes of discontinuities of $v$ outside $X_{\infty}$ die out at an exponential rate and are not newly created by the dynamics of $F$.

Proposition 8.2. There exists $\varepsilon \in(0,1)$ such that for all $s=\sigma+i b,|\sigma|<\varepsilon,|b| \geq \max \{4 \pi / D, 2\}$, and all $v \in B V(Y)$ such that $\left(H_{\sigma, m}\right)$ holds for some $m \geq 1$, there exists a pair $\left(u_{m n_{0}}, w_{m n_{0}}\right) \in$ $\mathcal{C}_{b}$ such that

$$
\left\|\tilde{\mathcal{L}}_{s}^{m n_{0}} v-w_{m n_{0}}\right\|_{\infty} \leq 2 C_{10} \rho^{-m n_{0}}|b|\|v\|_{\infty} \text { and }\left\|w_{m n_{0}}\right\|_{\infty} \leq\|v\|_{\infty} .
$$

The above result will allow us to prove

Lemma 8.3. There exist $\varepsilon \in(0,1)$ and $\beta \in(0,1)$ such that for all $s=\sigma+i b,|\sigma|<\varepsilon$, $|b| \geq \max \{4 \pi / D, 1\}$ and for all $m \geq 1$,

$$
\left\|\tilde{\mathcal{L}}_{s}^{3 m n_{0}} v\right\|_{b} \leq(1+|b|)^{-1} \operatorname{Var}_{Y}\left(\tilde{\mathcal{L}}_{s}^{3 m n_{0}} v\right)+\left(2 C_{10} \rho^{-m n_{0}}|b|+\beta^{m}\right)\|v\|_{\infty} .
$$

for all $v \in B V(Y)$ satisfying $\left(H_{\sigma, m}\right)$. 
Proof of Proposition 8.2. Let $v \in \mathrm{BV}(Y)$ be arbitrary and take $r=m n_{0}$ (this is a multiple of $k$ because $n_{0}$ is). Write $g_{r}=\tilde{\mathcal{L}}_{s}^{r} v$ and $\bar{g}_{r}=\tilde{\mathcal{L}}_{\sigma}^{r}|v|$; for every fixed $b \in \mathbb{R}$, they belong to $\operatorname{BV}(Y)$ as well by Proposition 3.5. Therefore $g_{r}$ has at most countably many discontinuity points, which we denote by $\left\{x_{i}\right\}_{i \in \mathbb{N}}$. Assume throughout this proof that $g_{r}$ is continuous from the right; this can be achieved by adjusting $g_{r}$ at $\left\{x_{i}\right\}_{i \in \mathbb{N}}$, so it has no effect on the $L^{p}$-norm for any $p \in[1, \infty]$.

To estimate the jump-size $\left|a_{i}\right|$ of $g_{r}$ at $x_{i} \in X_{j}^{\prime}$ for some $j \leq r$, we note that this discontinuity is created by non-onto branches of $F^{r}$, and there exist $y \in X_{1}^{\prime}$ and an inverse branch $\tilde{h} \in \mathcal{H}_{j-1}$ such that $y_{i}=\tilde{h}\left(x_{i}\right)$. The jump-size of $\tilde{\mathcal{L}}_{s}^{r} v$ at $x_{i}$ can be expressed as a sum of $h \in \mathcal{H}_{r-(j-1)}$ which in the summand is composed with $\tilde{h}$. Then

$$
\begin{aligned}
\text { Size } & \tilde{\mathcal{L}}_{s}^{r} v\left(x_{i}\right) \leq \sum_{h \in \mathcal{H}_{r-(j-1)}}\left|(h \circ \tilde{h})^{\prime}\left(x_{i}\right)\right|\left|e^{s \varphi_{r-(j-1)} \circ h \circ \tilde{h}\left(x_{i}\right)+s \varphi_{j-1} \circ \tilde{h}\left(x_{i}\right)}\right| \frac{\left(f_{\sigma} v\right) \circ h \circ \tilde{h}\left(x_{i}\right)}{\lambda_{\sigma}^{r} f_{\sigma}\left(x_{i}\right)} \\
& =\sum_{h \in \mathcal{H}_{r-(j-1)}}\left|h^{\prime}\left(y_{i}\right)\right| e^{\sigma \varphi_{r-(j-1)} \circ h\left(y_{i}\right)} \frac{\left(f_{\sigma} v\right) \circ h\left(y_{i}\right)}{\lambda_{\sigma}^{r-(j-1)} f_{\sigma}\left(y_{i}\right)}\left|\tilde{h}^{\prime}\left(x_{i}\right)\right| e^{\sigma \varphi_{j-1} \circ \tilde{h}\left(x_{i}\right)} \frac{f_{\sigma}\left(y_{i}\right)}{\lambda_{\sigma}^{j-1} f_{\sigma}\left(x_{i}\right)} \\
& \leq\left(\sum_{h \in \mathcal{H}_{n-(j-1)}}\left|h^{\prime}\left(y_{i}\right)\right| e^{\sigma \varphi_{r-(j-1)} \circ h\left(y_{i}\right)} \frac{f_{\sigma} \circ h\left(y_{i}\right)}{\lambda_{\sigma}^{r-(j-1)} f_{\sigma}\left(y_{i}\right)}\right)\|v\|_{\infty} \rho^{-3(j-1)} \frac{\sup f_{\sigma}}{\inf f_{\sigma}} \\
& \leq\|v\|_{\infty} \rho^{3} \frac{\sup f_{\sigma}}{\inf f_{\sigma}} \rho^{-3 j} .
\end{aligned}
$$

where the sum in brackets in the penultimate line is 1 because $f_{\sigma}$ is an eigenfunction of $\mathcal{L}_{\sigma}$.

For $r>k$, let $Q_{r}$ be an interval partition of $Y$ refining $\mathcal{P}_{r}$ such that $\frac{1}{2} \rho^{-r}<\operatorname{Leb}\left(I_{r}\right)<2 \rho^{-r}$ for every $I_{r} \in Q_{r}$. In fact, by adjusting $Q_{r}$ by an arbitrary small amount if necessary, we can assume that $g_{r}$ and $\bar{g}_{r}$ are continuous at every point in $\partial I_{r} \backslash X_{r}, I_{r} \in Q_{r}$. Construct $w_{r}$ and $u_{r}$ to be affine on each $(p, q)=I_{r} \in Q_{r}$ such that

$$
\lim _{x \downarrow p} w_{r}(x)=\lim _{x \downarrow p} g_{r}(x) \quad \text { and } \quad \lim _{x \uparrow q} w_{r}(x)=\lim _{x \uparrow q} g_{r}(x)
$$

and similarly

$$
\lim _{x \downarrow p} u_{r}(x)=\lim _{x \downarrow p} \bar{g}_{r}(x) \quad \text { and } \quad \lim _{x \uparrow q} u_{r}(x)=\lim _{x \uparrow q} \bar{g}_{r}(x) .
$$

Then $w_{r}$ and $u_{r}$ are continuous on $Y \backslash X_{r}$ and as $\bar{g}_{r} \geq\left|g_{r}\right|$, it is immediate that $u_{r} \geq\left|w_{r}\right|$ on $Y$. The main estimate now concerns the oscillation

$$
\operatorname{Osc}_{I_{r}} g_{r}=\operatorname{Osc}_{I_{r}}\left(\sum_{h \in \mathcal{H}_{r}, I_{r} \subset \operatorname{dom}(h)} \frac{e^{s \varphi_{r} \circ h}\left|h^{\prime}\right|}{\lambda_{\sigma}^{r} f_{\sigma}}\left(f_{\sigma} v\right) \circ h\right) \quad \text { for } I_{r} \in Q_{r},
$$

which we will split into five terms similar to the proof of the invariance of the cone.

The term with $\left|h^{\prime}\right|$ is bounded above by $C_{1} e^{C_{1}} \operatorname{Leb}\left(I_{r}\right) \sup _{x \in I_{r}} \tilde{\mathcal{L}}_{\sigma}^{r}|v|$ as in (7.4).

The term with $e^{s \varphi_{n} \circ h}$ is bounded above by $(1+|\sigma|) e^{\sigma C_{2}^{\prime}} C_{2}^{\prime}|b| \operatorname{Leb}\left(I_{r}\right) \sup _{x \in I_{r}} \tilde{\mathcal{L}}_{\sigma}^{r}|v|$ as in (7.6).

The term with $1 / f_{\sigma}$ is bounded above, by combining (7.11) and (7.12), by

$$
C_{6} \operatorname{Leb}\left(I_{r}\right) \sup _{x \in I_{r}} \tilde{\mathcal{L}}_{\sigma}^{r}|v|+C_{7} \operatorname{Leb}\left(I_{r}\right) \sum_{j>r} \rho^{-j} \sum_{x \in X_{j}^{\prime} \cap I_{r}} \tilde{\mathcal{L}}_{\sigma}^{r}|v|(x) .
$$

Here the second term is bounded by $C_{7} N_{1} \frac{\rho^{-r}}{\rho-1} \sup _{x \in I_{r}} \tilde{\mathcal{L}}_{\sigma}^{r}|v| \leq 2 C_{7} \frac{N_{1}}{\rho-1} \operatorname{Leb}\left(I_{r}\right) \sup _{x \in I_{r}} \tilde{\mathcal{L}}_{\sigma}^{r}|v|$, where we recall that $\# X_{j}^{\prime} \leq N_{1}$ for all $j \geq 1$.

The term with $f_{\sigma} \circ h$ is bounded above, by combining (7.8) and (7.9) and arguing as in the previous case, by

$$
C_{6} \rho_{0}^{-r} \operatorname{Leb}\left(I_{r}\right) \sup _{x \in I_{r}} \tilde{\mathcal{L}}_{\sigma}^{r}|v|+C_{7} \sum_{j>r} \rho^{-j} \sum_{x \in X_{j}^{\prime} \cap I_{r}} \tilde{\mathcal{L}}_{\sigma}^{r}|v|(x) \leq\left(C_{6} \rho_{0}^{-r}+2 C_{7} \frac{N}{\rho-1}\right) \operatorname{Leb}\left(I_{r}\right) \sup _{x \in I_{r}} \tilde{\mathcal{L}}_{\sigma}^{r}|v| .
$$


The term with $v \circ h$ : First we treat the case $\sigma \geq 0$. By Lemma C.2 (which also gives a lower bound $r_{0}$ for $r$ )

$$
\|v\|_{1} \leq \frac{K_{1}}{\operatorname{Leb}\left(I_{r}\right)} \int_{F^{-r}\left(I_{r}\right)}|v| d \text { Leb } \quad \text { for all } I_{r} \in Q_{r}
$$

where $K_{1}=6 e^{C_{1}} / \eta$. Recall that $\left(H_{\sigma, m}\right)$ holds with $C_{11}>1$ as defined in (8.1). Compute that

$$
\begin{aligned}
& \sum_{\substack{h \in \mathcal{H}_{r} \\
I_{r} \subset \operatorname{dom}(h)}}\left(\sup _{x \in I_{r}} \frac{\left|e^{s \varphi_{r} \circ h}\right|\left|h^{\prime}\right|}{\lambda_{\sigma}^{r} f_{\sigma}} f_{\sigma} \circ h\right) \operatorname{Osc}_{I_{r}}(v \circ h) \leq \rho^{-3 r} \frac{\sup f_{\sigma}}{\inf f_{\sigma}} \sum_{\substack{h \in \mathcal{H}_{r} \\
I_{r} \subset \operatorname{dom}(h)}} \operatorname{Osc}_{h\left(I_{r}\right)} v \\
& \leq \rho^{-3 r} \frac{\sup f_{\sigma}}{\inf f_{\sigma}} \operatorname{Var}_{F^{-r}\left(I_{r}\right)} v \leq 2 \rho^{-2 r} \operatorname{Leb}\left(I_{r}\right) \frac{\sup f_{\sigma}}{\inf f_{\sigma}} \operatorname{Var}_{Y} v \\
& \leq 2 \rho^{-2 r} \operatorname{Leb}\left(I_{r}\right) \frac{\sup f_{\sigma}}{\inf f_{\sigma}} C_{11}|b|^{2} \rho^{r} \int_{Y}|v| d \text { Leb } \\
& \leq 2 C_{11}|b|^{2} K_{1} \rho^{-r} \frac{\sup f_{\sigma}}{\inf f_{\sigma}} \int_{F^{-r}\left(I_{r}\right)}|v| d \text { Leb } \\
& \leq 2 C_{11}|b|^{2} K_{1} \rho^{-r}\left(\frac{\sup f_{\sigma}}{\inf f_{\sigma}}\right)^{2} \sum_{\substack{h \in \mathcal{H}_{r} \\
I_{r} \subset \operatorname{dom}(h)}} \int_{I_{r}} \frac{\left|h^{\prime}\right|}{f_{\sigma}}\left(f_{\sigma}|v|\right) \circ h d \text { Leb. }
\end{aligned}
$$

Because $\sigma \geq 0$, we can continue as

$$
\begin{aligned}
& \sum_{\substack{h \in \mathcal{H}_{r} \\
I_{r} \subset \operatorname{dom}(h)}}\left(\sup _{x \in I_{r}} \frac{\left|e^{s \varphi_{r} \circ h}\right|\left|h^{\prime}\right|}{\lambda_{\sigma}^{r} f_{\sigma}} f_{\sigma} \circ h\right) \operatorname{Osc}_{I_{r}}(v \circ h) \\
& \leq 2 C_{11}|b|^{2} K_{1} \rho^{-r} \lambda_{\sigma}^{r}\left(\frac{\sup f_{\sigma}}{\inf f_{\sigma}}\right)^{2} \sum_{\substack{h \in \mathcal{H}_{r} \\
I_{r} \subset \operatorname{dom}(h)}} \int_{I_{r}} \frac{e^{\sigma \varphi_{r} \circ h}\left|h^{\prime}\right|}{\lambda_{\sigma}^{r} f_{\sigma}}\left(f_{\sigma}|v|\right) \circ h d \text { Leb } \\
& \leq 2 C_{11}|b|^{2} K_{1} \rho^{-r} \lambda_{\sigma}^{r}\left(\frac{\sup f_{\sigma}}{\inf f_{\sigma}}\right)^{2} \operatorname{Leb}\left(I_{r}\right) \sup _{x \in I_{r}} \tilde{\mathcal{L}}_{\sigma}^{r}|v| .
\end{aligned}
$$

Since $\rho>\lambda_{\sigma}$, we obtain the upper bound $\operatorname{Leb}\left(I_{r}\right) \sup _{x \in I_{r}} \tilde{\mathcal{L}}_{\sigma}^{r}|v|$ by taking $r$ sufficiently large.

Now we treat the case $\sigma<0$. By Lemma C.2 applied to $e^{\sigma \varphi_{r}} v$ (and with the same lower bound $r_{0}$ for $r$ as before)

$$
\left\|e^{\sigma \varphi_{r}} v\right\|_{1} \leq \frac{K_{1}}{\operatorname{Leb}\left(I_{r}\right)} \int_{F^{-r}\left(I_{r}\right)}\left|e^{\sigma \varphi_{r}} v\right| d \text { Leb } \quad \text { for all } I_{r} \in Q_{r} .
$$

Note that

$$
\begin{aligned}
& \sum_{\substack{h \in \mathcal{H}_{r} \\
I_{r} \subset \operatorname{dom}(h)}}\left(\sup _{x \in I_{r}} \frac{\left|e^{s \varphi_{r} \circ h}\right|\left|h^{\prime}\right|}{\lambda_{\sigma}^{r} f_{\sigma}} f_{\sigma} \circ h\right) \operatorname{Osc}_{I_{r}}(v \circ h) \\
& \leq e^{\varepsilon C_{2}^{\prime}} \sum_{\substack{h \in \mathcal{H}_{r} \\
I_{r} \subset \operatorname{dom}(h)}}\left(\sup _{x \in I_{r}} \frac{\left|h^{\prime}\right|}{\lambda_{\sigma}^{r} f_{\sigma}} f_{\sigma} \circ h\right) \operatorname{Osc}_{I_{r}}\left(\left(e^{\sigma \varphi_{r}} v\right) \circ h\right) \\
& \leq e^{\varepsilon C_{2}^{\prime}} \lambda_{\sigma}^{-r} \frac{\sup f_{\sigma}}{\inf f_{\sigma}} \rho_{0}^{-r} \operatorname{Osc}_{I_{r}}\left(\left(e^{\sigma \varphi_{r}} v\right) \circ h\right) .
\end{aligned}
$$


Estimating the oscillation as in the case $\sigma \geq 0$, and using $\left(H_{\sigma, m}\right)$, we find the upper bound

$$
\begin{aligned}
& \sum_{\substack{h \in \mathcal{H}_{r} \\
I_{r} \subset \operatorname{dom}(h)}}\left(\sup _{x \in I_{r}} \frac{\left|e^{s \varphi_{r} \circ h}\right|\left|h^{\prime}\right|}{\lambda_{\sigma}^{r} f_{\sigma}} f_{\sigma} \circ h\right) \operatorname{Osc}_{I_{r}}(v \circ h) \\
& \leq 2 e^{\varepsilon C_{2}^{\prime}} C_{11}|b|^{2} K_{1} \rho^{-3 r}\left(\frac{\sup f_{\sigma}}{\inf f_{\sigma}}\right)^{2} \sum_{\substack{h \in \mathcal{H}_{r} \\
I_{r} \subset \operatorname{dom}(h)}} \int_{I_{r}} \frac{e^{\sigma \varphi_{r} \circ h}\left|h^{\prime}\right|}{\lambda_{\sigma}^{r} f_{\sigma}}\left(f_{\sigma}|v|\right) \circ h d \text { Leb } \\
& \leq 2 e^{\varepsilon C_{2}^{\prime}} C_{11}|b|^{2} K_{1} \rho^{-3 r}\left(\frac{\sup f_{\sigma}}{\inf f_{\sigma}}\right)^{2} \operatorname{Leb}\left(I_{r}\right) \sup _{x \in I_{r}} \tilde{\mathcal{L}}_{\sigma}^{r}|v| .
\end{aligned}
$$

By taking $r$ sufficiently large, we obtain again the upper bound $\operatorname{Leb}\left(I_{r}\right) \sup _{x \in I_{r}} \tilde{\mathcal{L}}_{\sigma}^{r}|v|$, and this finishes the case $\sigma<0$.

Putting all terms together,

$$
\operatorname{Osc}_{I_{r}} g_{r} \leq C_{10}|b| \operatorname{Leb}\left(I_{r}\right) \sup _{I_{r}} \tilde{\mathcal{L}}_{\sigma}^{r}|v|,
$$

and since $w_{r}$ is an affine interpolation of $g_{r}$, with the same limit values at all points $x_{i} \in X_{r}$,

$$
\left\|g_{r}-w_{r}\right\|_{\infty} \leq C_{10}|b| \operatorname{Leb}\left(I_{r}\right) \sup _{I_{r}} \tilde{\mathcal{L}}_{\sigma}^{r}|v| \leq 2 C_{10}|b| \rho^{-r}\|v\|_{\infty}
$$

Also, since $w_{r}$ is an affine interpolation of $g_{r}$, we have $\left\|w_{r}\right\| \leq\left\|g_{r}\right\|_{\infty} \leq\|v\|_{\infty}$.

We still need to complete the argument why $\left(u_{r}, w_{r}\right) \in \mathcal{C}_{b}$. By (8.3), the affine function $\left.w_{r}\right|_{I_{r}}$ has slope $C_{10}|b| \sup _{I_{r}} \tilde{\mathcal{L}}_{\sigma}^{r}|v|=C_{10}|b| \sup _{I_{r}}\left|u_{r}\right|$. This means that for every subinterval $I \subset I_{r}$, we also have

$$
\operatorname{Osc}_{I} w_{r} \leq C_{10}|b| \operatorname{Leb}(I) \sup _{I} u_{r} .
$$

If on the other hand, $I$ intersects several contiguous $I_{r} \in Q_{r}$ (but is contained in an atom of $\mathcal{P}_{k}$ ), then we have to include the jump-sizes of discontinuity points at $\partial I_{r}$ as well. But since $Q_{r}$ refines $\mathcal{P}_{r}$ and $g_{q}$ is continuous at all boundary points $q \in \partial I_{r} \backslash X_{r}$, and the jump-sizes of $g_{r}$ and $w_{r}$ coincide at every $x_{i} \in X_{j}^{\prime}$ (and decrease exponentially in $j$ by (8.2)) we conclude that

$$
\operatorname{Osc}_{I} w_{r} \leq C_{10}|b| \operatorname{Leb}(I) \sup _{I} u_{r}+C_{8} E_{I}\left(u_{r}\right) .
$$

This shows that $\left(u_{r}, w_{r}\right) \in \mathcal{C}_{b}$, as required.

Proof of Lemma 8.3. For $m \geq 1$ let $\left(w_{m n_{0}}, u_{m n_{0}}\right) \in \mathcal{C}_{b}$ be as in the statement of Proposition 8.2. Let $v \in \mathrm{BV}$. Using the definition of \|\|$_{b}$ norm,

$$
\begin{aligned}
\left\|\tilde{\mathcal{L}}_{s}^{3 m n_{0}} v\right\|_{b} & =(1+|b|)^{-1} \operatorname{Var}_{Y}\left(\tilde{\mathcal{L}}_{s}^{3 m n_{0}} v\right)+\left\|\tilde{\mathcal{L}}_{s}^{3 m n_{0}} v\right\|_{1} \\
& \leq(1+|b|)^{-1} \operatorname{Var}_{Y}\left(\tilde{\mathcal{L}}_{s}^{3 m n_{0}} v\right)+\left\|\tilde{\mathcal{L}}_{s}^{2 m n_{0}}\left(\tilde{\mathcal{L}}_{s}^{m n_{0}} v-w_{m n_{0}}\right)\right\|_{1}+\left\|\tilde{\mathcal{L}}_{s}^{2 m n_{0}} w_{n}\right\|_{1} \\
& \leq(1+|b|)^{-1} \operatorname{Var}_{Y}\left(\tilde{\mathcal{L}}_{s}^{3 m n_{0}} v\right)+2 C_{10} \rho^{-m n_{0}}|b|\|v\|_{\infty}+\beta^{m}\left\|w_{m n_{0}}\right\|_{\infty},
\end{aligned}
$$

where in the last inequality we have used Proposition 8.2 and Lemma 8.1. The conclusion follows since $\left\|w_{m n_{0}}\right\|_{\infty} \leq\|v\|_{\infty}$ (as in the statement of Proposition 8.2).

\subsection{Completing the argument}

In this section we complete the proof of Theorem 2.3 via a couple of lemmas.

Lemma 8.4. There exist $\varepsilon \in(0,1), A>0$ and $\gamma_{1} \in(0,1)$ such that for all $s=\sigma+i b,|\sigma|<\varepsilon$, $|b| \geq \max \{4 \pi / D, 2\}$ and for all $m \geq A \log (1+|b|)$,

$$
\left\|\tilde{\mathcal{L}}_{s}^{3 m n_{0}} v\right\|_{b} \leq \gamma_{1}^{3 m}\|v\|_{b}
$$

for all $v \in B V(Y)$ satisfying $\left(H_{\sigma, m}\right)$. 
Proof. First, we estimate $(1+|b|)^{-1} \operatorname{Var}_{Y}\left(\tilde{\mathcal{L}}_{s}^{3 m n_{0}} v\right)$. For $m \in \mathbb{N}$, recall from Proposition 8.2 and Lemma 8.1 that

$$
\begin{aligned}
\left\|\tilde{\mathcal{L}}_{s}^{2 m n_{0}} v\right\|_{1} & \leq\left\|\tilde{\mathcal{L}}_{s}^{m n_{0}}\left(\tilde{\mathcal{L}}_{s}^{m n_{0}} v-w_{m n_{0}}\right)\right\|_{1}+\left\|\tilde{\mathcal{L}}_{s}^{m n_{0}} w_{m n_{0}}\right\|_{1} \\
& \leq\left\|\tilde{\mathcal{L}}_{s}^{m n_{0}}\left(\tilde{\mathcal{L}}_{s}^{m n_{0}} v-w_{m n_{0}}\right)\right\|_{\infty}+\beta^{m}\left\|w_{m n_{0}}\right\|_{\infty} \\
& \leq 2 C_{10} \rho^{-m n_{0}}\|v\|_{\infty}+\beta^{m}\|v\|_{\infty} \leq 4 \beta^{m}\|v\|_{\infty}
\end{aligned}
$$

where we used $C_{10} \rho^{-m n_{0}} \leq 2 \beta^{m}$. By Proposition 3.5 (which is allowed since $n_{0}$ is a multiple of $k$ ) and recalling that $\Lambda_{\sigma}:=\lambda_{2 \sigma}^{1 / 2} / \lambda_{\sigma} \geq 1$, we compute

$$
\begin{aligned}
\operatorname{Var}_{Y}\left(\tilde{\mathcal{L}}_{s}^{3 m n_{0}} v\right) & \leq \rho^{-m n_{0}} \operatorname{Var}_{Y}\left(\tilde{\mathcal{L}}_{s}^{2 m n_{0}} v\right)+c(1+|b|) \Lambda_{\sigma}^{m n_{0}}\left(\left\|\tilde{\mathcal{L}}_{s}^{2 m n_{0}} v\right\|_{1}\left\|\tilde{\mathcal{L}}_{s}^{2 m n_{0}} v\right\|_{\infty}\right)^{1 / 2} \\
& \leq \rho^{-m n_{0}} \operatorname{Var}_{Y}\left(\tilde{\mathcal{L}}_{s}^{2 m n_{0}} v\right)+2 c(1+|b|) \Lambda_{\sigma}^{m n_{0}} \beta^{m / 2}\|v\|_{\infty} \\
& \leq \rho^{-m n_{0}} \operatorname{Var}_{Y}\left(\tilde{\mathcal{L}}_{s}^{2 m n_{0}} v\right)+2 c(1+|b|) \Lambda_{\sigma}^{m n_{0}} \beta^{m / 2}\left(\operatorname{Var}_{Y} v+\|v\|_{1}\right)
\end{aligned}
$$

where in the last inequality we have used $\|v\|_{\infty} \leq \operatorname{Var}_{Y} v+\|v\|_{1}$. Also by Proposition 3.5,

$$
\begin{aligned}
\operatorname{Var}_{Y}\left(\tilde{\mathcal{L}}_{s}^{2 m n_{0}} v\right) & \leq \rho^{-2 m n_{0}} \operatorname{Var}_{Y} v+c(1+|b|) \Lambda_{\sigma}^{2 m n_{0}}\|v\|_{\infty} \\
& \leq \rho^{-2 m n_{0}} \operatorname{Var}_{Y} v+c(1+|b|) \Lambda_{\sigma}^{2 m n_{0}}\left(\operatorname{Var}_{Y} v+\|v\|_{1}\right) .
\end{aligned}
$$

Plugging the above inequality into (8.4) we get

$$
\operatorname{Var}_{Y}\left(\tilde{\mathcal{L}}_{s}^{3 m n_{0}} v\right) \leq \rho^{-3 m n_{0}} \operatorname{Var}_{Y} v+c(1+|b|)\left(\rho^{-m n_{0}} \Lambda_{\sigma}^{2 m n_{0}}+2 \Lambda_{\sigma}^{m n_{0}} \beta^{m / 2}\right)\left(\operatorname{Var}_{Y} v+\|v\|_{1}\right) .
$$

Multiplying this $(1+|b|)^{-1}$ and inserting it in Lemma 8.3 (which relies on the assumption $\left(H_{\sigma, m}\right)$ ) gives

$$
\begin{aligned}
\left\|\tilde{\mathcal{L}}_{s}^{3 m n_{0}} v\right\|_{b} \leq & (1+|b|)^{-1} \rho^{-3 m n_{0}} \operatorname{Var}_{Y} v+c\left(\rho^{-m n_{0}} \Lambda_{\sigma}^{2 m n_{0}}+2 \Lambda_{\sigma}^{m n_{0}} \beta^{m / 2}\right)\left(\operatorname{Var}_{Y} v+\|v\|_{1}\right) \\
& +\left(2 C_{10} \rho^{-m n_{0}}|b|+\beta^{m}\right)\left(\operatorname{Var}_{Y} v+\|v\|_{1}\right) .
\end{aligned}
$$

Hence,

$$
\begin{aligned}
\left\|\tilde{\mathcal{L}}_{s}^{3 m n_{0}} v\right\|_{b} \leq & (1+|b|)^{-1}\left(\rho^{-3 m n_{0}}+(1+|b|)\left(c \Lambda_{\sigma}^{2 m n_{0}} \rho^{-m n_{0}}\right.\right. \\
& \left.\left.+2 c \Lambda_{\sigma}^{m n_{0}} \beta^{m / 2}+2 C_{10}|b| \rho^{-m n_{0}}+\beta^{m}\right)\right) \operatorname{Var}_{Y} v \\
& +\left(c \Lambda_{\sigma}^{2 m n_{0}} \rho^{-m n_{0}}+2 c \Lambda_{\sigma}^{m n_{0}} \beta^{m / 2}+2 C_{10}|b| \rho^{-m n_{0}}+\beta^{m}\right)\|v\|_{1} \\
\leq & (1+|b|)^{2}\left(2 C_{10}+c\right)\left(\Lambda_{\sigma}^{2 m n_{0}} \rho^{-m n_{0}}+\Lambda_{\sigma}^{m n_{0}} \beta^{m / 2}\right)\|v\|_{b} .
\end{aligned}
$$

Let $A>0$ be so large that $\gamma_{1}:=\max \left\{\Lambda_{\sigma}^{2 n_{0}} \rho^{-1}, \Lambda_{\sigma}^{n_{0}} \beta^{1 / 2}\right\} \exp \left(\frac{6 \log \left(2 C_{0}+c\right)}{A}\right)<1$. Then $(1+$ $|b|)^{2}\left(2 C_{10}+c\right)\left(\Lambda_{\sigma}^{2 m n_{0}} \rho^{-m n_{0}}+\Lambda_{\sigma}^{m n_{0}} \beta^{m / 2}\right)<\gamma_{1}^{m}$ for all $m>A \log (1+|b|)$, and the conclusion follows.

To complete the proof of Theorem 2.3 we still need to deal with BV functions violating $\left(H_{\sigma, m}\right)$.

Lemma 8.5. There exist $\varepsilon \in(0,1)$ and $\gamma_{2} \in(0,1)$ such that for all $s=\sigma+i b,|\sigma|<\varepsilon$, $|b| \geq \max \{4 \pi / D, 2\}$ and for all $m \geq 1$,

$$
\left\|\tilde{\mathcal{L}}_{s}^{m n_{0}} v\right\|_{b} \leq \gamma_{2}^{m}\|v\|_{b}
$$

for all $v \in B V(Y)$ violating $\left(H_{\sigma, m}\right)$.

Proof. By continuity in $\sigma, 1 \leq \Lambda_{\sigma}<\rho^{1 / 2}$ for all $|\sigma|$ sufficiently small. Then clearly also $\gamma_{2}:=$ $\Lambda_{\sigma}^{n_{0}} \rho^{-n_{0} / 2}<1$. We first treat the case $\sigma \geq 0$, so by assumption, $\operatorname{Var}_{Y} v>C_{11}|b|^{2} \rho^{m n_{0}}\|v\|_{1}$. 
Using Proposition 3.5 (which is allowed since $n_{0}$ is a multiple of $k$ ), we compute that

$$
\begin{aligned}
\operatorname{Var}_{Y}\left(\tilde{\mathcal{L}}_{s}^{m n_{0}} v\right) & \leq \rho^{-m n_{0}} \operatorname{Var}_{Y} v+c(1+|b|) \Lambda_{\sigma}^{m n_{0}}\left(\|v\|_{1}\|v\|_{\infty}\right)^{1 / 2} \\
& \leq \rho^{-m n_{0}} \operatorname{Var}_{Y}(v)+c(1+|b|) \Lambda_{\sigma}^{m n_{0}}\left(\|v\|_{1}\left(\operatorname{Var}_{Y} v+\|v\|_{1}\right)^{1 / 2}\right. \\
& \leq \rho^{-m n_{0}} \operatorname{Var}_{Y}(v)+c(1+|b|) \Lambda_{\sigma}^{m n_{0}}\left(\frac{\rho^{-m n_{0}}}{C_{11}|b|^{2}} \operatorname{Var}_{Y} v\left(\operatorname{Var}_{Y} v+\frac{\rho^{-m n_{0}}}{C_{11}|b|^{2}} \operatorname{Var}_{Y} v\right)\right)^{1 / 2} \\
& \leq \rho^{-m n_{0}} \operatorname{Var}_{Y} v+\frac{c}{C_{11}^{1 / 2}} \frac{\sqrt{65}}{8} \frac{1+|b|}{|b|} \Lambda_{\sigma}^{m n_{0}} \rho^{-m n_{0} / 2} \operatorname{Var}_{Y} v \\
& \leq\left(\rho^{-m n_{0}}+\frac{1}{8 K_{2}} \frac{3 \sqrt{65}}{16} \Lambda_{\sigma}^{m n_{0}} \rho^{-m n_{0} / 2}\right) \operatorname{Var}_{Y} v,
\end{aligned}
$$

where we have used $C_{11}|b|^{2}>64$ and abbreviated $K_{2}:=\frac{\sup f_{\sigma}}{\inf f_{\sigma}} \frac{\sup f_{0}}{\inf f_{0}}$. Therefore

$$
(1+|b|)^{-1} \operatorname{Var}_{Y}\left(\tilde{\mathcal{L}}_{s}^{m n_{0}} v\right) \leq(1+|b|)^{-1} \frac{1}{4 K_{2}} \gamma_{2}^{m} \operatorname{Var}_{Y} v
$$

for $m$ sufficiently large. By (A.4) at the end of the proof of Proposition 3.5,

$$
\left\|\tilde{\mathcal{L}}_{\sigma}^{m n_{0}}|v|\right\|_{1} \leq \Lambda_{\sigma}^{m n_{0}} \frac{\sup f_{\sigma}}{\inf f_{\sigma}}\left(\frac{\sup f_{2 \sigma}}{\inf f_{2 \sigma}}\right)^{1 / 2}\left(\|v\|_{\infty}\|v\|_{1}\right)^{1 / 2} .
$$

Note that $\left\|\tilde{\mathcal{L}}_{s}^{m n_{0}} v\right\|_{1} \leq\left\|\tilde{\mathcal{L}}_{\sigma}^{m n_{0}}|v|\right\|_{1}$. so we have

$$
\begin{aligned}
\left\|\tilde{\mathcal{L}}_{s}^{m n_{0}} v\right\|_{1} & \leq \Lambda_{\sigma}^{m n_{0}} \frac{\sup f_{\sigma}}{\inf f_{\sigma}}\left(\frac{\sup f_{2 \sigma}}{\inf f_{2 \sigma}}\right)^{1 / 2}\left(\left(\operatorname{Var}_{Y} v+\|v\|_{1}\right)\|v\|_{1}\right)^{1 / 2} \\
& \leq \Lambda_{\sigma}^{m n_{0}} \frac{\sup f_{\sigma}}{\inf f_{\sigma}}\left(\frac{\sup f_{2 \sigma}}{\inf f_{2 \sigma}}\right)^{1 / 2}\left(\left(1+\frac{\rho^{-m n_{0}}}{C_{11}|b|^{2}}\right) \frac{\rho^{-m n_{0}}}{C_{11}|b|^{2}}\right)^{1 / 2} \operatorname{Var}_{Y} v \\
& \leq \frac{\sup f_{\sigma}}{\inf f_{\sigma}}\left(\frac{\sup f_{2 \sigma}}{\inf f_{2 \sigma}}\right)^{1 / 2} \frac{\sqrt{65}}{8} C_{11}^{-1 / 2}|b|^{-1} \Lambda_{\sigma}^{m n_{0}} \rho^{-m n_{0} / 2} \operatorname{Var}_{Y} v
\end{aligned}
$$

The choice of $C_{11}$ gives that $\frac{\sup f_{\sigma}}{\inf f_{\sigma}}\left(\frac{\sup f_{2 \sigma}}{\inf f_{2 \sigma}}\right)^{1 / 2}<C_{11}^{1 / 2} / 8 K_{2}$. Hence, the choice of $\gamma_{2}$ gives $\left\|\tilde{\mathcal{L}}_{s}^{m n_{0}} v\right\|_{1} \leq \frac{1}{4 K_{2}}(1+|b|)^{-1} \gamma_{2}^{m} \operatorname{Var}_{Y} v$. Together, $\left\|\tilde{\mathcal{L}}_{s}^{m n_{0}}\right\|_{b} \leq \frac{1}{2 K_{2}}(1+|b|)^{-1} \gamma_{2}^{m} \operatorname{Var}_{Y} v$.

Now if $\sigma<0$, then the assumption is $\operatorname{Var}_{Y}\left(e^{\sigma \varphi_{m n_{0}}} v\right)>C_{11}|b|^{2} \rho^{m n_{0}}\left\|e^{\sigma \varphi_{m n_{0}}} v\right\|_{1}$. The above computation gives

$$
\left\|\tilde{\mathcal{L}}_{s}^{m n_{0}} v\right\|_{b} \leq \frac{\sup f_{\sigma}}{\inf f_{\sigma}} \frac{\sup f_{0}}{\inf f_{0}}\left\|\tilde{\mathcal{L}}_{i b}^{m n_{0}}\left(e^{\sigma \varphi_{m n_{0}}} v\right)\right\|_{b} \leq \frac{1}{2}(1+|b|)^{-1} \gamma_{2}^{m}\left(2 \operatorname{Var}_{Y} v+\|v\|_{1}\right),
$$

where we have used (since $\sigma<0)$ that $\operatorname{Var}_{Y}\left(e^{\sigma \varphi_{m n_{0}}} v\right) \leq \operatorname{Var}_{Y} v+\|v\|_{\infty} \leq 2 \operatorname{Var}_{Y} v+\|v\|_{1}$. Therefore $\left\|\tilde{\mathcal{L}}_{s}^{m n_{0}}\right\|_{b} \leq(1+|b|)^{-1} \gamma_{2}^{m}\|v\|_{b}$ and this proves the lemma.

Proof of Theorem 2.3. Let $\varepsilon \in(0,1)$ be such that the conclusion of Lemmas 8.4, 8.5 and Proposition 3.5 hold, and take $\gamma=\max \left\{\gamma_{1}^{1 / 2}, \gamma_{2}^{1 / 2}\right\}$. Let $|\sigma|<\varepsilon, n \in \mathbb{N}$ and $v \in \operatorname{BV}(Y)$ be arbitrary. Recall that $|b| \geq \max \{4 \pi / D, 2\}$. Let $A$ be the constant used in Lemma 8.4; without loss of generality, we can assume that $A \log |b|>3 n_{0}$. By the proof of Proposition 3.5 (see also Remark A.1), there is $A^{\prime}$ such that the operator norm

$$
\left\|\tilde{\mathcal{L}}_{s}^{n^{\prime}}\right\|_{b} \leq A^{\prime}(1+|b|) \quad \text { for all }|\sigma|<\varepsilon, b \in \mathbb{R}, n^{\prime} \in \mathbb{N} .
$$

Take

$$
n \geq 2 \max \left\{\frac{A}{n_{0}} \log (1+|b|), \log \left(\Lambda_{\sigma}^{-1} \frac{\sup f_{\sigma}}{\inf f_{\sigma}} A^{\prime}(1+|b|)\right)\right\} .
$$

Because the contraction in Lemmas 8.4 and 8.5 happen at different time steps, we carry out the following algorithm: 
1. Let $m_{0} \in \mathbb{N}$ be maximal such that $3 m_{0} n_{0} \leq n$. If $m_{0}<A \log (1+|b|)$, then continue with Step 4, otherwise continue with Step 2.

2. If $v$ satisfies $\left(H_{\sigma, m_{0}}\right)$, then $\left\|\tilde{\mathcal{L}}_{s}^{3 m_{0} n_{0}} v\right\|_{b} \leq \gamma^{6 m_{0}}\|v\|_{b}$ by Lemma 8.4 , and we continue with Step 4.

If $v$ does not satisfy $\left(H_{\sigma, m_{0}}\right)$, then $\left\|\tilde{\mathcal{L}}_{s}^{m_{0} n_{0}} v\right\|_{b} \leq \gamma^{2 m_{0}}\|v\|_{b}$ by Lemma 8.5. Let $v_{1}=$ $\tilde{\mathcal{L}}_{s}^{m_{0} n_{0}} v$ and let $m_{1} \in \mathbb{N}$ be maximal such that $3 m_{1} n_{0} \leq n-m_{0} n_{0}$.

If $m_{1}<A \log |b|$, then continue with Step 4, otherwise continue with Step 3.

3. If $v_{1}$ satisfies $\left(H_{\sigma, m_{1}}\right)$, then $\left\|\tilde{\mathcal{L}}_{s}^{3 m_{1} n_{0}} v_{1}\right\|_{b} \leq \gamma^{6 m_{0}}\|v\|_{b}$ by Lemma 8.4. Therefore

$\left\|\tilde{\mathcal{L}}_{s}^{\left(3 m_{1}+m_{0}\right) n_{0}} v\right\|_{b}=\left\|\tilde{\mathcal{L}}_{s}^{3 m_{1} n_{0}} v_{1}\right\|_{b} \leq \gamma^{6 m_{1}}\left\|v_{1}\right\|_{b}=\gamma^{3 m_{1}}\left\|\tilde{\mathcal{L}}_{s}^{3 m_{1} n_{0}} v\right\|_{b} \leq \gamma^{6 m_{1}+2 m_{0}}\|v\|_{b}$,

and we continue with Step 4.

If $v_{1}$ does not satisfies $\left(H_{\sigma, m_{1}}\right)$, then $\left\|\tilde{\mathcal{L}}_{s}^{m_{1} n_{0}} v_{1}\right\|_{b} \leq \gamma^{2 m_{0}}\left\|v_{1}\right\|_{b}$ by Lemma 8.5. Let $v_{2}=$ $\tilde{\mathcal{L}}_{s}^{m_{1} n_{0}} v_{1}$ and let $m_{2} \in \mathbb{N}$ be maximal such that $3 m_{2} n_{0} \leq n-\left(m_{0}+m_{1}\right) n_{0}$ and repeat Step 3. Each time we pass through Step 3, we introduce the next integer $m_{i}$ and $v_{i}=\tilde{\mathcal{L}}_{s}^{m_{i-1}} v_{i-1}$. As soon as $m_{i}<A \log (1+|b|)$ we continue with Step 4.

4. Let $p=p(v)$ be the number of times that this algorithm passes through Step 3. Note that $p<\infty$ because each time Step 3 is taken, $n-\left(m_{0}+m_{1}+\cdots+m_{i}\right) n_{0}$ decreases by a factor $2 / 3$. Thus we find a sequence $\left(m_{i}\right)_{i=0}^{p}$ and we can define

$$
M_{p}=M_{p}(v)=\left\{\begin{array}{l}
m_{0}+\cdots+m_{p-1}+3 m_{p}, \quad \text { or } \\
m_{0}+\cdots+m_{p-1}+m_{p},
\end{array}\right.
$$

depending on whether $v_{p-1}=\tilde{\mathcal{L}}_{s}^{\left(m_{0}+\cdots+m_{p-1}\right) n_{0}} v$ satisfies $\left(H_{\sigma, m_{p-1}}\right)$ or not. In either case we have $n-M_{p} n_{0}<A \log (1+|b|)$ and $\left\|\tilde{\mathcal{L}}_{s}^{M_{p} n_{0}} v\right\|_{b} \leq \gamma^{2 M_{p}}\|v\|_{b}$.

By (8.5), we have for all $v \in \mathrm{BV}(Y)$

$$
\left\|\tilde{\mathcal{L}}_{s}^{n} v\right\|_{b}=\left\|\tilde{\mathcal{L}}_{s}^{n-M_{p} n_{0}}\left(\tilde{\mathcal{L}}_{s}^{M_{p} n_{0}} v\right)\right\|_{b} \leq\left\|\tilde{\mathcal{L}}_{s}^{n-M_{p} n_{0}}\right\|_{b}\left\|\tilde{\mathcal{L}}_{s}^{M_{p} n_{0}} v\right\|_{b} \leq A^{\prime}(1+|b|) \gamma^{2 M_{p}}\|v\|_{b} .
$$

Also $\left\|\mathcal{L}_{s}^{n} v\right\|_{b} \leq \lambda_{\sigma}^{-1} \frac{\sup f_{\sigma}}{\inf f_{\sigma}}\left\|\tilde{\mathcal{L}}_{s}^{n} v\right\|_{b}$. Therefore, using $n-M_{p} n_{0}<A \log |b|$,

$$
\begin{aligned}
\left\|\mathcal{L}_{s}^{n} v\right\|_{b} & \leq \lambda_{\sigma}^{-1} \frac{\sup f_{\sigma}}{\inf f_{\sigma}} A^{\prime}(1+|b|) \gamma^{2 M_{p}}\|v\|_{b} \\
& \leq \lambda_{\sigma}^{-1} \frac{\sup f_{\sigma}}{\inf f_{\sigma}} A^{\prime}(1+|b|) \gamma^{(-A \log |b|) / n_{0}} \gamma^{2 n}\|v\|_{b} \\
& \leq \lambda_{\sigma}^{-1} \frac{\sup f_{\sigma}}{\inf f_{\sigma}} A^{\prime}(1+|b|) \gamma^{n / 2} \gamma^{(-A \log |b|) / n_{0}} \gamma^{n / 2} \gamma^{n}\|v\|_{b} \leq \gamma^{n}\|v\|_{b},
\end{aligned}
$$

since $n$ is chosen large enough as in (8.6). This completes the proof.

\section{A Proof of Proposition 3.5}

Proof of Proposition 3.5. Fix $k$ and $\varepsilon$ such that the assumptions of the proposition hold. First, we provide the argument for $n=k$; the conclusion for $n$ a multiple of $k$ will follow by a standard iteration argument. We note that for each $a \in \alpha^{k}$ the interval $F^{k}(a)=\left[p_{a}, q_{a}\right]$ is the domain of an inverse branch $h \in \mathcal{H}_{k}$, which is a contracting diffeomorphism.

Compute that

$$
\begin{aligned}
\operatorname{Var}_{Y} \tilde{\mathcal{L}}_{s}^{k} v & \leq \frac{1}{\lambda_{\sigma}^{k}} \frac{1}{\inf f_{\sigma}} \operatorname{Var}\left(\sum_{h \in \mathcal{H}_{k}} e^{s \varphi_{k} \circ h}\left|h^{\prime}\right|\left(f_{\sigma} v\right) \circ h\right)+\frac{1}{\lambda_{\sigma}^{k}} \operatorname{Var}\left(\frac{1}{f_{\sigma}}\right)\left\|\sum_{h \in \mathcal{H}_{k}} e^{s \varphi_{k} \circ h}\left|h^{\prime}\right|\left(f_{\sigma} v\right) \circ h\right\|_{\infty} \\
& \leq \frac{Q}{\lambda_{\sigma}^{k}} \operatorname{Var}\left(\sum_{h \in \mathcal{H}_{k}} e^{s \varphi_{k} \circ h}\left|h^{\prime}\right|\left(f_{\sigma} v\right) \circ h\right)+\operatorname{Var}\left(\frac{1}{f_{\sigma}}\right)\left\|\frac{1}{\lambda_{\sigma}^{k}} \sum_{h \in \mathcal{H}_{k}} e^{s \varphi_{k} \circ h}\left|h^{\prime}\right|\left(f_{\sigma} v\right) \circ h\right\|_{1} \\
& \leq \frac{Q}{\lambda_{\sigma}^{k}} \operatorname{Var}\left(\mathcal{L}_{s}^{k}\left(f_{\sigma} v\right)\right)+\operatorname{Var}\left(\frac{1}{f_{\sigma}}\right) \sup f_{\sigma} \int \tilde{\mathcal{L}}_{\sigma}^{k}|v| d \text { Leb, }
\end{aligned}
$$


where we abbreviated $Q:=\frac{1}{\inf f_{\sigma}}+\operatorname{Var}\left(\frac{1}{f_{\sigma}}\right)$.

We estimate the first term in the above equation. Since $v \in \mathrm{BV}(Y), v$ is differentiable Lebesgue-a.e. on $Y$ and we let $d v$ denote the generalized derivative; so, for $[p, q] \subset Y$, we have $\operatorname{Var}_{Y}\left(1_{[p, q]} v\right) \leq \int_{p}^{q}|d v|+|v(p)|+|v(q)|$ (see, for instance, [9]).

$$
\begin{aligned}
\frac{1}{\lambda_{\sigma}^{k}} \operatorname{Var}\left(\mathcal{L}_{s}^{k}\left(f_{\sigma} v\right)\right) \leq & \sum_{h \in \mathcal{H}_{k}}\left(\int_{\operatorname{dom}(h)} \frac{\left|d\left(e^{s \varphi_{k} \circ h}\left|h^{\prime}\right|\left(f_{\sigma} v\right) \circ h\right)\right|}{\lambda_{\sigma}^{k}}\right. \\
& \left.+\frac{\left|e^{s \varphi_{k} \circ h}\right|\left|h^{\prime}\right|\left(f_{\sigma}|v|\right) \circ h}{\lambda_{\sigma}^{k}}\left(p_{a}\right)+\frac{\left|e^{s \varphi_{k} \circ h}\right|\left|h^{\prime}\right|\left(f_{\sigma}|v|\right) \circ h}{\lambda_{\sigma}^{k}}\left(q_{a}\right)\right) \\
\leq & 2 \sum_{h \in \mathcal{H}_{k}} \int_{\operatorname{dom}(h)}\left|d\left(\frac{e^{s \varphi_{k} \circ h}\left|h^{\prime}\right|\left(f_{\sigma} v\right) \circ h}{\lambda_{\sigma}^{k}}\right)\right| \\
& +2 \sum_{h \in \mathcal{H}_{k}} \inf _{\left.p_{a}, q_{a}\right]}\left|\frac{e^{s \varphi_{k} \circ h(x)}\left|h^{\prime}(x)\right|\left(f_{\sigma} v\right) \circ h(x)}{\lambda_{\sigma}^{k}}\right|=: J_{1}+J_{2} .
\end{aligned}
$$

First, by the finite image property, $c_{0}:=\min _{a \in \alpha^{k}}\left(q_{a}-p_{a}\right)>0$ for our fixed $k$. Therefore

$$
J_{2} \leq \frac{2}{\min _{a \in \alpha^{k}}\left(q_{a}-p_{a}\right)} \sum_{h \in \mathcal{H}_{k}} \int_{F^{k}(a)} \frac{e^{\sigma \varphi_{k} \circ h(x)}\left|h^{\prime}(x)\right|\left(f_{\sigma}|v|\right) \circ h(x)}{\lambda_{\sigma}^{k}} \leq \frac{2 \sup f_{\sigma}}{c_{0}} \int_{Y} \tilde{\mathcal{L}}_{\sigma}^{k}|v| d \text { Leb. }
$$

We split the term $J_{1}$ in (A.2) into three terms

$$
\sum_{h \in \mathcal{H}_{k}} \int_{\operatorname{dom}(h)}\left|d\left(\frac{e^{s \varphi_{k} \circ h}\left|h^{\prime}\right|\left(f_{\sigma} v\right) \circ h}{\lambda_{\sigma}^{k}}\right)\right| \leq I_{1}+I_{2}+I_{3}
$$

corresponding to which factor of $\frac{e^{s \varphi_{k} \circ h}\left|h^{\prime}\right|\left(f_{\sigma} v\right) \circ h}{\lambda_{\sigma}^{k}}$ the derivative is taken of.

For $I_{1}$ : Taking $m=k$ in (7.5)

$$
\begin{aligned}
I_{1} & :=|\sigma+i b| \sum_{h \in \mathcal{H}_{k}} \int_{\operatorname{dom}(h)} \frac{e^{\sigma \varphi_{k} \circ h}\left(\varphi_{k} \circ h\right)^{\prime}\left|h^{\prime}\right|\left(f_{\sigma}|v|\right) \circ h}{\lambda_{\sigma}^{k}} d \text { Leb } \\
& \leq C_{2}^{\prime}|\varepsilon+b| \sup f_{\sigma} \int_{Y} \tilde{\mathcal{L}}_{\sigma}^{k}|v| d \text { Leb. }
\end{aligned}
$$

For $I_{2}$ : Taking $n=k$ in (7.3),

$$
I_{2}=\sum_{h \in \mathcal{H}_{k}} \int_{\operatorname{dom}(h)} \frac{e^{\sigma \varphi_{k} \circ h}\left|h^{\prime \prime}\right|\left(f_{\sigma}|v|\right) \circ h}{\lambda_{\sigma}^{k}} d \text { Leb } \leq C_{1} \sup f_{\sigma} \int_{Y} \tilde{\mathcal{L}}_{\sigma}^{k}|v| d \text { Leb. }
$$

For $I_{3}$ : Due to (3.3) and using a change of coordinates,

$$
\begin{aligned}
I_{3} & =\sum_{h \in \mathcal{H}_{k}} \int_{\operatorname{dom}(h)}\left|\frac{e^{\sigma \varphi_{k} \circ h}\left|h^{\prime}\right|^{2} d\left(f_{\sigma} v\right) \circ h}{\lambda_{\sigma}^{k}}\right| d \text { Leb } \leq \rho^{-3 k} \sum_{h \in \mathcal{H}_{k}} \int_{a}\left|d\left(f_{\sigma} v\right)\right| d \text { Leb } \\
& \leq \rho^{-3 k} \int_{Y}\left|d\left(f_{\sigma} v\right)\right| d \operatorname{Leb}=\rho^{-3 k} \operatorname{Var}_{Y}\left(f_{\sigma} v\right) \leq \rho^{-3 k} \sup f_{\sigma} \operatorname{Var}_{Y} v+\rho^{-3 k} \operatorname{Var}_{Y} f_{\sigma}\|v\|_{\infty} \\
& \leq \rho^{-3 k}\left(\sup f_{\sigma}+\operatorname{Var}_{Y} f_{\sigma}\right) \operatorname{Var}_{Y} v+\rho^{-3 k} \operatorname{Var}_{Y} f_{\sigma} \int_{Y}|v| d \text { Leb },
\end{aligned}
$$

where in the last inequality we have used $\|v\|_{\infty} \leq \operatorname{Var}_{Y} v+\int|v| d$ Leb. Putting these together,

$$
\begin{aligned}
\frac{1}{\lambda_{\sigma}^{k}} \operatorname{Var}\left(\mathcal{L}_{s}^{k}\left(f_{\sigma} v\right)\right) \leq & \rho^{-3 k}\left(\sup f_{\sigma}+\operatorname{Var}_{Y} f_{\sigma}\right) \operatorname{Var}_{Y} v \\
& +\rho^{-3 k} \operatorname{Var}_{Y} f_{\sigma} \int_{Y}|v| d \text { Leb }+\left(c_{1}+C_{2}^{\prime}|b|\right) \sup f_{\sigma} \int_{Y} \tilde{\mathcal{L}}_{\sigma}^{k}|v| d \text { Leb },
\end{aligned}
$$


where $c_{1}=2 c_{0}^{-1}+C_{1}+C_{2}^{\prime} \varepsilon$ and $C_{2}^{\prime}$ is as in (7.5). This together with (A.1) implies that

$$
\begin{aligned}
\operatorname{Var}_{Y} \tilde{\mathcal{L}}_{s}^{k} v \leq & \rho^{-3 k} Q\left(\sup f_{\sigma}+\operatorname{Var}_{Y} f_{\sigma}\right) \operatorname{Var}_{Y} v+\rho^{-3 k} \operatorname{Var}_{Y} f_{\sigma} \int_{Y}|v| d \text { Leb } \\
& +\left(c_{1}+\operatorname{Var}\left(\frac{1}{f_{\sigma}}\right)+C_{2}^{\prime}|b|\right) \sup f_{\sigma} \int_{Y} \tilde{\mathcal{L}}_{\sigma}^{k}|v| d \text { Leb. }
\end{aligned}
$$

Given our choice of $\varepsilon, c_{2}:=\operatorname{Var}\left(\frac{1}{f_{\sigma}}\right)<\infty$. By (2.7), $c:=\rho^{-2 k} Q\left(\sup f_{\sigma}+\operatorname{Var}_{Y} f_{\sigma}\right)<1$ and $\rho^{-3 k} \operatorname{Var}_{Y} f_{\sigma}<1$. Therefore

$$
\operatorname{Var}_{Y} \tilde{\mathcal{L}}_{s}^{k} v \leq \rho^{-k} \operatorname{Var}_{Y} v+\int_{Y}|v| d \operatorname{Leb}+\left(c_{1}+c_{2}+C_{2}^{\prime}|b|\right) \sup f_{\sigma} \int_{Y} \tilde{\mathcal{L}}_{\sigma}^{k}|v| d \text { Leb. }
$$

For $n \geq 1$ arbitrary, we estimate $\int_{Y} \tilde{\mathcal{L}}_{\sigma}^{n k}|v| d$ Leb applying Cauchy-Schwartz. First, note that

$$
\int_{Y} \tilde{\mathcal{L}}_{\sigma}^{n k}|v| d \operatorname{Leb} \leq\left(\int_{Y}\left(\tilde{\mathcal{L}}_{\sigma}^{n k}|v|\right)^{2} d \mathrm{Leb}\right)^{1 / 2} .
$$

Recall that $\Lambda_{\sigma}=\frac{\lambda_{2 \sigma}^{1 / 2}}{\lambda_{\sigma}}$. Then

$$
\begin{aligned}
& \int\left(\tilde{\mathcal{L}}_{\sigma}^{n k}|v|\right)^{2} d \text { Leb }=\int\left(\lambda_{\sigma}^{n k} f_{\sigma}\right)^{-2}\left(\sum_{h \in \mathcal{H}_{n k}} e^{\sigma \varphi_{n k} \circ h}\left|h^{\prime}\right|\left(f_{\sigma}|v|\right) \circ h\right)^{2} d \text { Leb } \\
& =\int\left(\lambda_{\sigma}^{n k} f_{\sigma}\right)^{-2}\left(\sum_{h \in \mathcal{H}_{n k}}\left(e^{\sigma \varphi_{n k} \circ h}\left|h^{\prime}\right|^{1 / 2}\left(f_{\sigma}|v|\right)^{1 / 2} \circ h\right)\left(\left|h^{\prime}\right|^{1 / 2}\left(f_{\sigma}|v|\right)^{1 / 2} \circ h\right)\right)^{2} d \text { Leb } \\
& \leq \lambda_{\sigma}^{-2 n k}\left(\inf f_{\sigma}^{2}\right)^{-1} \int\left(\sum_{h \in \mathcal{H}_{n k}} e^{2 \sigma \varphi_{n k} \circ h}\left|h^{\prime}\right|\left(f_{\sigma}|v|\right) \circ h\right)\left(\sum_{h \in \mathcal{H}_{n k}}\left|h^{\prime}\right|\left(f_{\sigma}|v|\right) \circ h\right) d \text { Leb } \\
& \leq \Lambda_{\sigma}^{2 n k}\left(\frac{\sup f_{\sigma}}{\inf f_{\sigma}}\right)^{2} \frac{\sup f_{2 \sigma}}{\inf f_{2 \sigma}}\|v\|_{\infty} \int\left(\sum_{h \in \mathcal{H}_{n k}} \frac{e^{2 \sigma \varphi_{n k} \circ h}}{\Lambda_{2 \sigma}^{n k} f_{2 \sigma}}\left|h^{\prime}\right| f_{2 \sigma} \circ h\right)\left(\sum_{h \in \mathcal{H}_{n k}}\left|h^{\prime}\right||v| \circ h\right) d \text { Leb } \\
& \leq \Lambda_{\sigma}^{2 n k}\left(\frac{\sup f_{\sigma}}{\inf f_{\sigma}}\right)^{2} \frac{\sup f_{2 \sigma}}{\inf f_{2 \sigma}}\|v\|_{\infty}\|v\|_{1} .
\end{aligned}
$$

Thus,

$$
\int_{Y} \tilde{\mathcal{L}}_{\sigma}^{n k}|v| d \operatorname{Leb} \leq \Lambda_{\sigma}^{n k} \frac{\sup f_{\sigma}}{\inf f_{\sigma}}\left(\frac{\sup f_{2 \sigma}}{\inf f_{2 \sigma}}\right)^{1 / 2}\left(\|v\|_{\infty}\|v\|_{1}\right)^{1 / 2} .
$$

The above together with (A.3) implies that

$$
\begin{aligned}
\operatorname{Var}_{Y} \tilde{\mathcal{L}}_{s}^{n k} v & \leq \rho^{-k} \operatorname{Var}_{Y} \tilde{\mathcal{L}}_{s}^{(n-1) k} v+\left(1+c_{1}+c_{2}+C_{2}^{\prime}|b|\right) \Lambda_{\sigma}^{n k} \frac{\sup f_{\sigma}}{\inf f_{\sigma}}\left(\frac{\sup f_{2 \sigma}}{\inf f_{2 \sigma}}\right)^{1 / 2}\left(\|v\|_{\infty}\|v\|_{1}\right)^{1 / 2} \\
& \leq \rho^{-k} \operatorname{Var}_{Y} \tilde{\mathcal{L}}_{\sigma}^{(n-1) k} v+c_{3}(1+|b|) \Lambda_{\sigma}^{n k}\left(\|v\|_{\infty}\|v\|_{1}\right)^{1 / 2}
\end{aligned}
$$

$$
\operatorname{Var}_{Y} \tilde{\mathcal{L}}_{s}^{n k} v \leq \rho^{-n k} \operatorname{Var}_{Y} v+c(1+|b|) \Lambda_{\sigma}^{n k}\left(\|v\|_{\infty}\|v\|_{1}\right)^{1 / 2},
$$

for any $n \geq 1$, where $c:=c_{3} \sup f_{\sigma} \sum_{j=0}^{n-1}\left(\rho \Lambda_{\sigma}\right)^{-j k}$. This ends the proof.

Remark A.1. A similar, but much more simplified, argument to the one used in the proof of Proposition 3.5 shows that the non-normalized twisted transfer operator satisfies $\operatorname{Var}_{Y}\left(\mathcal{L}_{s}^{n} v\right) \leq$ $c_{1} \rho_{0}^{-n} \operatorname{Var}_{Y} v+c_{2}(1+|b|)\|v\|_{\infty}$, for all $n \geq 1$, some $\rho_{0}>1, c_{1}, c_{2}>0$, for all $b \in \mathbb{R}$ and all $|\sigma|<\varepsilon$, for any $\varepsilon \in(0,1)$.

Remark A.2. If $\sigma=0$, so when working on the imaginary axis, we can get the standard LasotaYorke inequality $\operatorname{Var}_{Y} \mathcal{L}_{i b}^{n} v \leq \rho^{-n} \operatorname{Var}_{Y} v+c_{4}(1+|b|)\|v\|_{1}$. 
Proof of Proposition 3.1. Take $\varepsilon=\varepsilon_{0}^{2}$. Without loss of generality, set $0 \leq\left|\sigma_{2}\right| \leq\left|\sigma_{1}\right|<\varepsilon$ and take $b \in \mathbb{R}$,

$$
\begin{aligned}
\left\|\mathcal{L}_{s\left(\sigma_{1}+i b_{1}\right.} v-\mathcal{L}_{\left(\sigma_{2}+i b_{2}\right.} v\right\|_{1} & =\int_{Y}\left|\sum_{h \in \mathcal{H}}\left(e^{\left(\sigma_{1}+i b_{1}\right) \varphi \circ h}-e^{\left(\sigma_{2}+i b_{2}\right) \varphi \circ h}\right)\right| h^{\prime}|v \circ h| d \text { Leb } \\
& \leq\|v\|_{\infty} \int_{Y} \sum_{h \in \mathcal{H}} e^{\sigma_{1} \varphi \circ h}\left|h^{\prime}\right|\left(1-e^{\left(\sigma_{2}-\sigma_{2}\right) \varphi \circ h}\right) d \text { Leb. }
\end{aligned}
$$

Because the function $x \mapsto e^{-\left(\varepsilon_{0}-\sigma_{1}\right) x} x$ assumes its maximum value $e^{-1}\left(\varepsilon_{0}-\sigma\right)^{-1}$ at $x=$ $\left(\varepsilon_{0}-\sigma\right)^{-1}$, we have

$$
e^{\sigma_{1} \varphi \circ h}\left(1-e^{\left(\sigma_{2}-\sigma_{2}\right) \varphi \circ h}\right) \leq e^{\varepsilon_{0} \varphi \circ h}\left|\sigma_{1}-\sigma_{2}\right| e^{-\left(\varepsilon_{0}-\sigma_{1}\right) \varphi \circ h} \varphi \circ h \leq \frac{e^{\varepsilon_{0} \varphi \circ h}}{e\left(\varepsilon_{0}-\sigma\right)} .
$$

Plugging this into the above, we find

$$
\int_{Y} \sum_{h \in \mathcal{H}}\left(e^{\sigma_{1} \varphi \circ h}-e^{\sigma_{2} \varphi \circ h}\right)\left|h^{\prime}\right| v \circ h d \operatorname{Leb} \leq \frac{\|v\|_{\infty}}{e\left(\varepsilon_{0}-\sigma\right)} \int_{Y} \sum_{h \in \mathcal{H}} e^{\varepsilon_{0} \varphi \circ h}\left|h^{\prime}\right| d \operatorname{Leb} \leq \frac{C_{3}\|v\|_{\infty}}{e\left(\varepsilon_{0}-\sigma\right)} .
$$

To estimate $\operatorname{Var}_{Y}\left(\mathcal{L}_{s\left(\sigma_{1}+i b_{1}\right.} v-\mathcal{L}_{\left(\sigma_{2}+i b_{2}\right.} v\right)$, we work as in the Proof of Proposition 3.5, and use the above estimate on the $L^{1}$-norm. As such we obtain

$\operatorname{Var}_{Y}\left(\mathcal{L}_{s\left(\sigma_{1}+i b_{1}\right.} v-\mathcal{L}_{\left(\sigma_{2}+i b_{2}\right.} v\right) \leq\left|\sigma_{1}-\sigma_{2}\right| \varepsilon_{0}^{-1}\left(C^{\prime} \operatorname{Var}_{Y} v+C^{\prime \prime}\|v\|_{\infty}\right) \leq C\left|\sigma_{1}-\sigma_{2}\right| \varepsilon_{0}^{-1}\|v\|_{\mathrm{BV}}$ for some $C>0$ as required.

\section{B Proofs of Lemmas 5.1 and 5.2}

Proof of Lemma 5.1. Recall that $f_{\sigma}$ is an eigenfunction for the non-normalized twisted transfer operator $\mathcal{L}_{\sigma}$, so $\frac{1}{\lambda_{\sigma}} \mathcal{L}_{\sigma}^{r} f_{\sigma}(x)=f_{\sigma}(x)$ for every $r \in \mathbb{N}$ and $x \in Y$. Therefore, for $r \in \mathbb{N}$ arbitrary, we have

$$
\begin{aligned}
\frac{1}{\lambda_{\sigma}^{r}} \mathcal{L}_{\sigma}^{r} 1(x) & =\frac{1}{\lambda_{\sigma}^{r}} \sum_{h \in \mathcal{H}_{r}, x \in \operatorname{dom}(h)}\left|h^{\prime}(x)\right| e^{\sigma \varphi_{r} \circ h(x)} \\
& \leq \sum_{h \in \mathcal{H}_{r}, x \in \operatorname{dom}(h)} \frac{\left|h^{\prime}(x)\right| e^{\sigma \varphi_{r} \circ h(x)} f_{\sigma} \circ h(x)}{\lambda_{\sigma}^{r} f_{\sigma}(x)} \frac{\sup f_{\sigma}}{\inf f_{\sigma}} \leq \frac{\sup f_{\sigma}}{\inf f_{\sigma}}
\end{aligned}
$$

for all $x \in Y$, and similarly $\frac{1}{\lambda_{\sigma}^{r}} \mathcal{L}_{\sigma}^{r} 1(x) \geq \frac{\inf f_{\sigma}}{\sup f_{\sigma}}$. Hence the Cesaro means converge to the fixed point with unit $L^{1}$-norm:

$$
\lim _{n \rightarrow \infty} \frac{1}{n} \sum_{r=0}^{n-1} \mathcal{L}_{\sigma}^{r} 1=\frac{f_{\sigma}}{\int_{Y} f_{\sigma} d \mathrm{Leb}} .
$$

If $x \notin X_{\infty}$, then $\mathcal{L}_{\sigma}^{r} 1$ is continuous at $x$ for all $r \in \mathbb{N}$, and so is $f_{\sigma}$. Now for $x \in X_{j}^{\prime}$ take $r \geq j$. The discontinuity of $\mathcal{L}_{\sigma}^{r} 1$ at $x \in X_{j}^{\prime}$ is created by non-onto branches of $F^{r}$, and there exist $y \in X_{1}^{\prime}$ and an inverse branch $\tilde{h} \in \mathcal{H}_{j-1}$ such that $y=\tilde{h}(x)$. The jump-size of $\tilde{\mathcal{L}}_{\sigma}^{r} 1$ at $x$ can be expressed as a sum of $h \in \mathcal{H}_{r-(j-1)}$ which in the summand is composed with $\tilde{h}$. Then, using (3.3) and also (B.1) for iterate $r-(j-1)$ to estimate the sum in brackets below:

$$
\begin{aligned}
\text { Size } \frac{1}{\lambda_{\sigma}^{r}} \mathcal{L}_{\sigma}^{r} 1(x) & \leq \frac{1}{\lambda_{\sigma}^{r}} \sum_{h \in \mathcal{H}_{r-(j-1)}, y \in \operatorname{dom}(h)}\left|(h \circ \tilde{h})^{\prime}(x)\right| e^{\sigma \varphi_{r-(j-1)} \circ h \circ \tilde{h}(x)+\sigma \varphi_{j-1} \circ \tilde{h}(x)} \\
& =\left(\sum_{h \in \mathcal{H}_{r-(j-1)}, y \in \operatorname{dom}(h)} \frac{\left|h^{\prime}(y)\right| e^{\sigma \varphi_{r-(j-1)} \circ h(y)}}{\lambda_{\sigma}^{r-(j-1)}}\right) \frac{\left|\tilde{h}^{\prime}(x)\right| e^{\sigma \varphi_{j-1} \circ \tilde{h}(x)}}{\lambda_{\sigma}^{j-1}} \\
& \leq \frac{\sup f_{\sigma}}{\inf f_{\sigma}} \rho^{-3(j-1)} .
\end{aligned}
$$


By taking the Cesaro limit we obtain statement 1. of the lemma for $C_{7}=\rho^{3} \frac{\sup f_{\sigma}}{\inf f_{\sigma}}$.

Now for statement 2. let $I \subset Y$ be an arbitrary interval, and let $J$ denote a component of $I \backslash X_{r}$. Note that if $h \in \mathcal{H}_{r}$ is such that $J \cap \operatorname{dom}(h) \neq \emptyset$, then $\operatorname{dom}(h) \supset J$. The oscillation $\operatorname{Osc}_{I}\left(\frac{1}{\lambda_{\sigma}^{r}} \mathcal{L}_{\sigma}^{r} 1\right)$ is bounded by the sum of jump-sizes of discontinuities in $I$ added to the sum of the oscillations $\operatorname{Osc}_{J}\left(\frac{1}{\lambda^{r}} \mathcal{L}_{\sigma}^{r} 1\right)$ on the components $J$ of $I \backslash X_{r}$. For the latter, we have using formulas (7.5), (7.3) and (B.1):

$$
\begin{aligned}
\operatorname{Osc}_{J}\left(\frac{1}{\lambda_{\sigma}^{r}} \mathcal{L}_{\sigma}^{r} 1\right) & \leq \frac{1}{\lambda_{\sigma}^{r}} \sum_{h \in \mathcal{H}_{r}} \int_{J \cap \operatorname{dom}(h)}\left|\left(e^{\sigma \varphi_{r} \circ h(\xi)}\left|h^{\prime}(\xi)\right|\right)^{\prime}\right| d \xi \\
& \leq \frac{1}{\lambda_{\sigma}^{r}} \sum_{h \in \mathcal{H}_{r}} \int_{J \cap \operatorname{dom}(h)}\left(|\sigma|\left|\left(\varphi_{r} \circ h\right)^{\prime}(\xi)\right| e^{\sigma \varphi_{r} \circ h(\xi)}+e^{\sigma \varphi_{r} \circ h(\xi)}\left|h^{\prime \prime}(\xi)\right|\right) d \xi \\
& \leq \int_{J} \sum_{h \in \mathcal{H}_{r}, J \cap \operatorname{dom}(h) \neq \emptyset} \frac{e^{\sigma \varphi_{r} \circ h(\xi)}\left|h^{\prime}(\xi)\right|}{\lambda_{\sigma}^{r}}\left(|\sigma| C_{2}^{\prime}+C_{1}\right) d \xi \\
& \leq\left(\varepsilon C_{2}^{\prime}+C_{1}\right) \int_{J} \frac{\sup f_{\sigma}}{\inf f_{\sigma}} d \xi=\left(\varepsilon C_{2}^{\prime}+C_{1}\right) \frac{\sup f_{\sigma}}{\inf f_{\sigma}} \operatorname{Leb}(J) .
\end{aligned}
$$

Recall from Remark 3.2 that $\frac{\sup f_{\sigma}}{\inf f_{\sigma}} \leq C_{5}$. Summing over all components $J$ of $I \backslash X_{r}$ gives

$$
\operatorname{Osc}_{I}\left(\frac{1}{\lambda_{\sigma}^{r}} \mathcal{L}_{\sigma}^{r} 1\right) \leq\left(\varepsilon C_{2}^{\prime}+C_{1}\right) C_{5} \operatorname{Leb}(I)+\rho^{3} C_{5} \sum_{j \leq r} \sum_{x \in X_{j}^{\prime} \cap I} \rho^{-3 j} .
$$

For the Cesaro limit, we get $\operatorname{Osc}_{I}\left(f_{\sigma}\right) \leq C_{6} \mu(I)+C_{7} E_{I}\left(f_{\sigma}\right)$ for $C_{6}=\left(\varepsilon C_{2}^{\prime}+C_{1}\right) C_{5}$ and $C_{7}=\rho^{3} C_{5}$ as required. This implies also the formula for $\operatorname{Osc}\left(1 / f_{\sigma}\right)$, adjusting the constants $C_{6}$ and $C_{7}$ if necessary.

Before stating the next lemma, we recall that $K=\min \{\operatorname{Leb}(F(a)): a \in \alpha\}$ and that $\delta_{0}=\frac{K\left(\rho_{0}-2\right)}{5 e^{C_{1} \rho_{0}}}$. Since $F$ is topologically mixing, there is $k_{1} \in \mathbb{N}$ such that $F^{k_{1}}(I) \supset Y$ for all intervals $I$ of length $\operatorname{Leb}(I) \geq \delta_{0}$.

Lemma B.1. There is $\eta_{1} \in(0,1)$ such that for every $z \in Y$ and $\tau>0$ the following property holds: For every $n \geq k_{1}+\frac{\log \left(2 K\left(\rho_{0}-2\right) /\left(e^{C_{1}} \rho_{0} \tau\right)\right)}{\log \left(\rho_{0} / 2\right)}$ and every interval $J$ of length $\operatorname{Leb}(J)>\tau$,

$$
\operatorname{Leb}\left(\bigcup_{\tilde{a} \in J_{z}} \tilde{a}\right) \geq \eta_{1} \operatorname{Leb}(J) \quad \text { for } J_{z}=\left\{\tilde{a} \in \alpha^{n}: \tilde{a} \subset J \text { and } z \in F^{n}(\tilde{a})\right\} .
$$

Proof. By the choice of $k_{1}$, there is a finite collection $\Omega$ of $k_{1}$-cylinders such that for each $z \in Y$ and each $I$ with $\operatorname{Leb}(I) \geq \delta_{0}$, there is $\omega \in \Omega, \omega \subset I$, such that $z \in F^{k_{1}}(\omega)$. Let $\gamma_{0}:=$ $\min \left\{\frac{\operatorname{Leb}(\omega)}{2 \delta_{0}}: \omega \in \Omega\right\}>0$.

For $y \in Y$, define $r_{j}(y)=d\left(F^{j}(y), \partial F^{j}(a)\right)$, where $a \in \alpha^{j}$ is the $j$-cylinder containing $y$. Take $J$ an arbitrary interval of length $\operatorname{Leb}(J) \geq \tau$, and define $Z_{\delta}^{j}=\left\{y \in J: r_{j}(y) \leq \delta\right\}$. We derive $\operatorname{Leb}\left(Z_{\delta}^{j+1}\right)$ from $\operatorname{Leb}\left(Z_{\delta}^{j}\right)$ as follows. If $a \in \alpha^{j}, W=F^{j}(a)$ and $a^{\prime} \in \alpha$ are such that $\partial W \cap a^{\prime} \neq \emptyset$, then the points $\left\{z \in F\left(W \cap a^{\prime}\right): d\left(z, \partial F\left(W \cap a^{\prime}\right)\right) \leq \delta\right\}$ pull back to at most two intervals in $W \cap a^{\prime}$ of combined length $\leq 2 \delta / \rho_{0}$, and this contributes $2 \operatorname{Leb}\left(Z_{\delta / \rho_{0}}^{j}\right)$ to $\operatorname{Leb}\left(Z_{\delta}^{j+1}\right)$. For the cylinders $a^{\prime} \in \alpha$ that are contained in $W$, we recall that $\operatorname{Leb}\left(F\left(a^{\prime}\right)\right) \geq K$. By the distortion bound from (2.2) we find $\operatorname{Leb}\left(Z_{\delta}^{j+1} \cap F^{-j}\left(a^{\prime}\right)\right) \leq \frac{2 e^{C_{1}} \delta}{K} \operatorname{Leb}(a)$. Combining this (and summing over all such $a$ ), we get the recursive relation $\operatorname{Leb}\left(Z_{\delta}^{j+1}\right) \leq 2 \operatorname{Leb}\left(Z_{\delta / \rho_{0}}^{j}\right)+$ $\frac{2 e^{C_{1}} \delta}{K} \operatorname{Leb}(J)$. This gives

$$
\operatorname{Leb}\left(Z_{\delta}^{j}\right) \leq 2^{j} \operatorname{Leb}\left(Z_{\delta / \rho_{0}^{j}}^{0}\right)+\frac{2 e^{C_{1}} \delta}{K} \sum_{i=0}^{j-1}\left(\frac{2}{\rho_{0}}\right)^{i} \leq\left(\left(\frac{2}{\rho_{0}}\right)^{j} \frac{\delta}{\operatorname{Leb}(J)}+\frac{2 e^{C_{1}} \rho_{0}}{K\left(\rho_{0}-2\right)} \delta\right) \operatorname{Leb}(J) .
$$


Take $\delta=\delta_{0}$ and $j \geq \frac{\log \left(10 \delta_{0} / \operatorname{Leb}(J)\right)}{\log \left(\rho_{0} / 2\right)}=\frac{\log \left(2 K\left(\rho_{0}-2\right) /\left(e^{C_{1}} \rho_{0} \tau\right)\right)}{\log \left(\rho_{0} / 2\right)}$ (so that $\left.\left(\frac{2}{\rho_{0}}\right)^{j} \frac{\delta_{0}}{\operatorname{Leb}(J)} \leq \frac{1}{10}\right)$. Then

$$
\operatorname{Leb}\left(y \in J: r_{j}(y) \geq \delta_{0}\right)=\operatorname{Leb}(J)-\operatorname{Leb}\left(Z_{\delta_{0}}^{j}\right) \geq \operatorname{Leb}(J)-\frac{1}{2} \operatorname{Leb}(J)=\frac{1}{2} \operatorname{Leb}(J) .
$$

Let $B_{j, J}$ be the collection of $a \in \alpha^{j}, a \subset J$ such that there is $y \in a$ with $r_{j}(y) \geq \delta_{0}$. This means by (B.2) that $\operatorname{Leb}\left(\cup_{a \in B_{j, J}} a\right) \geq \frac{1}{2} \operatorname{Leb}(J)$ and $1 \geq \operatorname{Leb}\left(F^{j}(a)\right) \geq 2 \delta_{0}$ for each $a \in B_{j, J}$. Take $z \in Y$ and $n=j+k_{1}$. It follows that there is an $n$-cylinder $\tilde{a} \subset a$ such that $F^{j}(\tilde{a})=\omega \in \Omega$ and $z \in F^{k_{1}}(\omega)$. By boundedness of distortion

$$
\frac{\operatorname{Leb}(\tilde{a})}{\operatorname{Leb}(a)} \geq e^{-C_{1}} \frac{\operatorname{Leb}\left(F^{j}(\tilde{a})\right.}{\operatorname{Leb}\left(F^{j}(a)\right)} \geq e^{-C_{1}} \frac{\operatorname{Leb}(\omega)}{2 \delta_{0}} \geq \gamma_{0} e^{-C_{1}} .
$$

Hence $\operatorname{Leb}\left(\cup_{a \in J_{z}} a\right) \geq \gamma_{0} e^{-C_{1}} \operatorname{Leb}\left(\cup_{a \in B_{n, J}} a\right) \geq \frac{\gamma_{0}}{2 e^{C_{1}}} \operatorname{Leb}(J)$, proving the lemma for $\eta_{1}:=$ $\frac{\gamma_{0}}{2 e^{C_{1}}}$.

Now we are ready for the proof of Lemma 5.2, which uses assumption (2.5).

Proof of Lemma 5.2. We will apply Lemma B.1 for $J=p$, an arbitrary element of $\mathcal{P}_{k}$. Set for $C_{9}=\eta_{1} e^{-C_{1}} / 2$. Assumption (2.5) gives $\operatorname{Leb}(p) \geq 12 \rho^{-k}$. Since $n=2 k$, we have $j:=n-k_{1} \geq$ $k$. Therefore $\left(\frac{2}{\rho_{0}}\right)^{j} \frac{\delta_{0}}{\operatorname{Leb}(p)} \leq \frac{2^{k} \rho^{-3 k} \delta_{0}}{12}<\frac{1}{12}$, and hence (B.2) implies that $\operatorname{Leb}\left(y \in p: r_{j}(y) \geq\right.$ $\left.\delta_{0}\right) \geq \frac{1}{2} \operatorname{Leb}(p)$.

Recall that $B_{j, p} \supset\left\{a \in \alpha^{j}: a \subset p, r_{j}(y) \geq \delta_{0}\right.$ for some $\left.y \in a\right\}$, so $F^{j}(a) \geq 2 \delta_{0}$ for each $a \in B_{j, p}$. In particular, such $a$ contains an $\tilde{a} \in \alpha^{n}$ such that $z \in F^{n}(\tilde{a})$, and $\operatorname{Leb}\left(\cup_{a \in B_{j, p}} \tilde{a}\right) \geq$ $\eta_{1} \operatorname{Leb}(p)$ with $\eta_{1}$ as in Lemma B.1. Let $B_{j, p}^{*}$ be a finite subcollection of $B_{j, p}$ such that $\operatorname{Leb}\left(\cup_{a \in B_{j, p}^{*}} \tilde{a}\right) \geq$ $\frac{2}{3} \eta_{1} \operatorname{Leb}(p)$, and let $h_{\tilde{a}}: F^{n}(\tilde{a}) \rightarrow \tilde{a}$ denote the corresponding inverse branches.

Using the continuity of $\sigma \mapsto \lambda_{\sigma}$ and $\sigma \mapsto e^{\sigma \varphi_{n} \circ h_{\tilde{a}}(z)}$ for all $a \in B_{j, p}^{*}, j \leq 4 k-k_{1}$ and $p \in \mathcal{P}_{k}$, we can choose $\varepsilon$ so small that $\frac{1}{\lambda_{\sigma}^{n}}\left|h_{\tilde{a}}^{\prime}(z)\right| e^{\sigma \varphi_{n} \circ h_{\tilde{a}}(z)} \geq \frac{3}{4}\left|h_{\tilde{a}}^{\prime}(z)\right|$ for all $a \in B_{j, p}^{*}$ and all $|\sigma|<\varepsilon$. Therefore

$$
\begin{aligned}
\frac{1}{\lambda_{\sigma}^{n}} \sum_{\substack{h \in \mathcal{H}_{n}, z \in \operatorname{dom}(h) \\
\text { range }(h) \subset p}}\left|h^{\prime}(z)\right| e^{\sigma \varphi_{n} \circ h(z)} & \geq \frac{1}{\lambda_{\sigma}^{n}} \sum_{a \in B_{j, p}^{*}}\left|h_{\tilde{a}}^{\prime}(z)\right| e^{\sigma \varphi_{n} \circ h_{\tilde{a}}(z)} \geq \frac{3}{4} \sum_{a \in B_{j, p}^{*}}\left|h_{\tilde{a}}^{\prime}(z)\right| \\
& \geq \frac{3}{4} \sum_{a \in B_{j, p}^{*}} e^{-C_{1}} \frac{\operatorname{Leb}(\tilde{a})}{\operatorname{Leb}\left(F^{n}(\tilde{a})\right)} \geq \frac{\eta_{1} \operatorname{Leb}(p)}{2 e^{C_{1}}} .
\end{aligned}
$$

This finishes the proof.

\section{A technical result for the proof of Proposition 8.2}

In this subsection we will use the generalised BV seminorm $\mathfrak{v a r}_{Y} v$ introduced by Keller [11] because it compares more easily with \|\|$_{1}$ than $\operatorname{Var}_{Y}$ does. To be precise, we define

$$
\mathfrak{v a r}_{Y} v=\sup _{0<\kappa<1} \frac{1}{\kappa} \int_{Y} \operatorname{Osc}\left(v, B_{\kappa}(x)\right) d \text { Leb, }
$$

where $\operatorname{Osc}\left(v, B_{\kappa}(x)\right)=\sup _{y, y^{\prime} \in B_{\kappa}(x)}\left|v(y)-v\left(y^{\prime}\right)\right|$ (also for complex-valued functions).

Lemma C.1. In dimension one, $\operatorname{Var}_{Y}$ and $\mathfrak{v a r}_{Y}$ are equivalent seminorms. More precisely, for all $v \in B V(Y)$ we have

$$
\frac{1}{2} \operatorname{Var}_{Y} v \leq \mathfrak{v a r}_{Y} v \leq 3 \operatorname{Var}_{Y} v
$$


Proof. [5, Lemma 1] states that $\operatorname{Var}_{Y} v \leq 2 \mathfrak{v a r}_{Y} v$. For the other inequality, choose $\kappa \in(0,1)$ and partition $Y$ into half-open intervals $J$ of length $|J| \leq \kappa$. For each such $J$, let $J^{\prime}$ and $J^{\prime \prime}$ denote its left and right neighbour. Then

$$
\begin{aligned}
\frac{1}{\kappa} \int_{Y} \operatorname{Osc}\left(v, B_{\kappa}(x)\right) d \operatorname{Leb} & =\frac{1}{\kappa} \sum_{J} \int_{J} \operatorname{Osc}\left(v, B_{\kappa}(x)\right) d \operatorname{Leb} \leq \frac{1}{\kappa} \sum_{J} \operatorname{Leb}(J) \operatorname{Osc}_{J \cup J^{\prime} \cup J^{\prime \prime}} v \\
& \leq \sum_{J} \operatorname{Osc}_{J \cup J^{\prime} \cup J^{\prime \prime}} v \leq 3 \operatorname{Var}_{Y} v .
\end{aligned}
$$

Both inequalities together prove (C.1).

Recall that $K:=\min \{|F(a)|: a \in \alpha\}$.

Lemma C.2. Let $v \in B V(Y)$ such that $\operatorname{Var}_{Y} v \leq K_{0}\|v\|_{1}$ for some $K_{0}>1$. Choose $\eta_{1} \in(0,1)$ such that Lemma B.1 holds and take $K_{1}=6 e^{C_{1}} / \eta_{1}$. Let

$$
r_{0}:=\max \left\{k, k_{1}+\left(\log \frac{108 K_{0} K\left(\rho_{0}-2\right)}{e_{1}^{C} \rho_{0}}\right) / \log \frac{\rho_{0}}{2}\right\} .
$$

Then for every $r>r_{0}$ and all $I_{r} \in Q_{r}$,

$$
\|v\|_{1} \leq \frac{K_{1}}{\operatorname{Leb}\left(I_{r}\right)} \int_{F^{-r}\left(I_{r}\right)}|v| d L e b .
$$

Proof of Lemma C.2. Fix $\kappa_{1}:=\left(18 K_{0}\right)^{-1}$. Since we assumed that $K_{1}>6 e^{C_{1}} / \eta_{1}$ we have $\left(1-\frac{4 e^{C_{1}}}{\eta_{1} K_{1}}\right) \geq 6 K_{0} \kappa_{1}$. Let $E$ be a partition of $Y$ into half-open intervals $J=[p, q)$ of length $\frac{\kappa_{1}}{3} \leq \operatorname{Leb}(J) \leq \frac{\kappa_{1}}{2}$. Next recall that $K:=\min \{|F(a)|: a \in \alpha\}$ and take $r>r_{0}$. Note that this $r_{0}$ is the bound from Lemma B.1 with $\tau=\kappa_{1} / 3=1 /\left(54 K_{0}\right)$.

We prove the lemma by contradiction, so assume that there exists $I_{r} \in Q_{r}$ such that $\|v\|_{1}>$ $\frac{K_{1}}{\operatorname{Leb}\left(I_{r}\right)} \int_{F^{-r}\left(I_{r}\right)}|v| d$ Leb. Define

$$
M\left(I_{r}\right)=\left\{J \in E: \int_{F^{-r}\left(I_{r}\right) \cap J}|v| d \operatorname{Leb} \leq \frac{2 \operatorname{Leb}\left(I_{r}\right)}{K_{1}} \int_{J}|v| d \operatorname{Leb}\right\} .
$$

If $\sum_{J \in M\left(I_{r}\right)} \int_{J}|v| d$ Leb $<\frac{1}{2}\|v\|_{1}$ (so $\sum_{J \notin M\left(I_{r}\right)} \int_{J}|v| d$ Leb $>\frac{1}{2}\|v\|_{1}$ ), then we have

$$
\begin{aligned}
\int_{F^{-r}\left(I_{r}\right)}|v| d \text { Leb } & \geq \sum_{J \notin M\left(I_{r}\right)} \int_{F^{-r}\left(I_{r}\right) \cap J}|v| d \text { Leb } \\
& >\frac{2 \operatorname{Leb}\left(I_{r}\right)}{K_{1}} \sum_{J \notin M\left(I_{r}\right)} \int_{J}|v| d \operatorname{Leb}>\frac{2 \operatorname{Leb}\left(I_{r}\right)}{K_{1}} \frac{1}{2} \int_{Y}|v| d \text { Leb },
\end{aligned}
$$

contradicting our choice of $I_{r}$. Therefore, it remains to deal with the case

$$
\sum_{J \in M\left(I_{r}\right)} \int_{J}|v| d \mathrm{Leb}>\frac{1}{2}\|v\|_{1}
$$

Recall that $e^{C_{1}}$ is a uniform distortion bound for the inverse branches of $F^{r}$. Let $z$ be the middle point of $I_{r}$ and $J_{z}=\left\{a \in \alpha^{r}: a \subset J, z \in F^{r}(a)\right\}$. This means in particular that $\frac{\operatorname{Leb}\left(F^{r}(a) \cap I_{r}\right.}{\operatorname{Leb}\left(F^{r}(a)\right)} \geq$ $\frac{1}{2} \operatorname{Leb}\left(I_{r}\right)$ for each $a \in J_{z}$. By Lemma B.1, $\operatorname{Leb}\left(\cup_{a \in J_{z}} a\right) \geq \eta_{1} \operatorname{Leb}(J)$. This gives

$$
\begin{aligned}
\int_{F^{-r}\left(I_{r}\right) \cap J}|v| d \operatorname{Leb} & \geq \inf _{J}|v| \operatorname{Leb}\left(F^{-r}\left(I_{r}\right) \cap J\right) \geq \inf _{J}|v| \sum_{a \in J_{z}} \operatorname{Leb}\left(F^{-r}\left(I_{r}\right) \cap a\right) \\
& \geq \inf _{J}|v| \sum_{a \in J_{z}} e^{-C_{1}} \frac{\operatorname{Leb}\left(F^{r}(a) \cap I_{r}\right)}{\operatorname{Leb}\left(F^{r}(a)\right)} \operatorname{Leb}(a) \\
& \geq \frac{\inf _{J}|v|}{2 e^{C_{1}}} \sum_{a \in J_{z}} \operatorname{Leb}(a) \operatorname{Leb}\left(I_{r}\right) \geq \frac{\eta_{1} \inf _{J}|v|}{2 e^{C_{1}}} \operatorname{Leb}(J) \operatorname{Leb}\left(I_{r}\right) .
\end{aligned}
$$


Hence for each $J \in M\left(I_{r}\right)$,

$$
\begin{aligned}
\operatorname{Leb}(J) \operatorname{Leb}\left(I_{r}\right) \inf _{J}|v| & \leq \frac{2 e^{C_{1}}}{\gamma} \int_{F^{-r}\left(I_{r}\right) \cap J}|v| d \operatorname{Leb} \\
& \leq \frac{4 e^{C_{1}}}{\eta_{1} K_{1}} \operatorname{Leb}\left(I_{r}\right) \int_{J}|v| d \operatorname{Leb} \leq \frac{4 e^{C_{1}}}{\eta_{1} K_{1}} \operatorname{Leb}(J) \operatorname{Leb}\left(I_{r}\right) \sup _{J}|v|
\end{aligned}
$$

and therefore $\inf _{J}|v| \leq \frac{4 e^{C_{1}}}{\eta_{1} K_{1}} \sup _{J}|v|$ and

$$
\operatorname{Osc}_{J} v \geq \operatorname{Osc}_{J}|v| \geq\left(1-\frac{4 e^{C_{1}}}{\eta_{1} K_{1}}\right) \sup _{J}|v| .
$$

Recall that by the choice of $\kappa_{1}, \kappa_{1}^{-1}\left(1-\frac{2 e^{C_{1}}}{\eta_{1} K_{1}}\right) \geq 6 K_{0}$. Bounding the sup from below using (C.3), we obtain

$$
\sup _{0<\kappa<1} \frac{1}{\kappa} \int_{J} \operatorname{Osc}\left(v, B_{\varepsilon}(x)\right) d \operatorname{Leb} \geq \operatorname{Leb}(J) \kappa_{1}^{-1}\left(1-\frac{4 e^{C_{1}}}{\eta_{1} K_{1}}\right) \sup _{J}|v| \geq 6 K_{0} \operatorname{Leb}(J) \sup _{J}|v| .
$$

By the second inequality in (C.1),

$$
\begin{aligned}
\operatorname{Var}_{Y} v & \geq \frac{1}{3} \mathfrak{v a r} \mathfrak{r}_{Y} v \geq \frac{1}{3 \kappa} \sum_{J \in E} \int_{J} \operatorname{Osc}\left(v, B_{\kappa}(x)\right) d \text { Leb } \\
& \geq \frac{1}{3} \sum_{J \in M\left(I_{r}\right)} 6 K_{0} \operatorname{Leb}(J) \sup _{J}|v| \geq 2 K_{0} \sum_{J \in M\left(I_{r}\right)} \int_{J}|v| d \text { Leb. }
\end{aligned}
$$

Finally (C.2) gives $\operatorname{Var}_{Y} v>K_{0} \int_{Y}|v| d$ Leb $=K_{0}\|v\|_{1}$. This contradicts the assumption of the lemma, completing the proof.

\section{Proof of Theorem 2.6}

The proof of Theorem 2.6 follows closely the argument used in [1, Proof of Theorem 2.1] with obvious required modifications. As in [1], the conclusion follows once we show that the Laplace transform $\hat{\rho}(s):=\hat{\rho}(s)(v, w):=\int_{0}^{\infty} e^{s t} \rho_{t}(v, w) d t$ behaves as described in the result below.

Lemma D.1. There exists $\varepsilon>0$ such that $\hat{\rho}(s)$ is analytic on $\{\Re s>\varepsilon\}$ for all $v \in F_{B V, 2}\left(Y^{\varphi}\right)$ and $w \in L^{\infty}\left(Y^{\varphi}\right)$. Moreover, there exists $C>0$ such that $|\hat{\rho}(s)| \leq C\left(1+|b|^{1 / 2}\right)\|v\|_{B V, 2}\|w\|_{\infty}$, for all $s=\sigma+i b$ with $\sigma \in\left[0, \frac{1}{2} \varepsilon\right]$.

The proof of Theorem 2.6 given Lemma D.1 is standard, relying on the formula $\rho_{t}(v, w)=$ $\int_{\Gamma} e^{-s t} \hat{\rho}(s) d s$, where $\Gamma=\{\Re s=\varepsilon / 2\}$; it goes, for instance, exactly the same as [1, Proof of Theorem 2.1] given [1, Lemma 2.17], so we omit this.

The proof of Lemma D.1 uses three ranges of $n$ and $b$ : i) $n \leq A \log |b|,|b| \geq 2$ with $A$ as in Theorem 2.3, ii) $|b| \geq \max \{4 \pi / D, 2\}$ and iii) $0<|b|<\max \{4 \pi / D, 2\}$. The first two regions go almost word by word as in [1, Lemma 2.17]. For the third region, the part of the proof in [1] where the standard form of Lasota-Yorke inequality of $\tilde{\mathcal{L}}_{s}$ is used doesn't apply (in our case $\left\|\tilde{\mathcal{L}}_{\sigma+i b}\right\|_{1}$ with $\sigma>0$ is not bounded). Instead, we use quasi-compactness of $\tilde{\mathcal{L}}_{i b}$ (i.e., $\sigma=0$ ) given by Remark A.2 and the continuity estimate of Proposition 3.1. These together ensure that the essential spectral radius of $\tilde{\mathcal{L}}_{s}$ is strictly less than 1 , and that the spectrum in a neighbourhood of 1 contains only isolated eigenvalues. The rest of the argument goes exactly as [1, Proof of Lemma 2.22], distinguishing between $b \neq 0$ the and $b=0$. In particular, proceeding as in [1, Proof of Lemma 2.22], we obtain the aperiodicity property and analyticity of the operator $Q_{i b}$ in the notation of [1, Proof of Lemma 2.22] in a neighborhood of $b$ for each $b \neq 0$. Also, in a neighborhood of $b=0$ we speak of the isolated eigenvalue $\lambda_{i b}$ (for the operator $\tilde{\mathcal{L}}_{i b}$ ) and corresponding spectral projection $P_{i b}$. Using again the continuity property of $\tilde{\mathcal{L}}_{s}$ given by Proposition 3.1, we can continue $\lambda_{s}$ and $P_{s}$ in a neighborhood of $s=0$. 
Acknowledgements: We would like to thank Ian Melbourne for valuable discussions and Tomas Persson for informing us about reference [2]. We are also grateful for the support the Erwin Schrödinger Institute in Vienna, where this paper was completed.

\section{References}

[1] V. Araújo, I. Melbourne, Exponential decay of correlations for non-uniformly hyperbolic flows with a $C^{1+\alpha}$ stable foliation. Preprint 2015 arXiv:1504.04316, to appear in Annales Henri Poincaré.

[2] M. Aspenberg, T. Persson, Shrinking targets in parametrised families. Preprint 2016 arXiv:1603.01116

[3] A. Avila, S. Gouëzel, J.-C. Yoccoz, Exponential mixing for the Teichmüller flow, Publ. Math. Inst. Hautes Études Sci. 104 (2006), 143-211.

[4] V. Baladi, B. Vallée, Exponential decay of correlations for surface semi-flows without finite Markov partitions, Proc. Amer. Math. Soc. 133 (2005), 865-874.

[5] J. Buzzi, G. Keller, Zeta functions and transfer operators for multidimensional piecewise affine and expanding maps, Ergod. Th. \& Dynam. Sys. 21 (2001), 690-716.

[6] O. Butterley, P. Eslami, Exponential mixing for skew products with discontinuities, Trans. of the AMS 369 (2017), 783-803.

[7] D. Dolgopyat, On the decay of correlations in Anosov flows, Ann. of Math. 147 (1998) 357-390.

[8] P. Eslami, Stretched-exponential mixing for $C^{1+\alpha}$ skew products with discontinuities, Ergod. Th. \& Dynam. Sys., published online July 2015.

[9] E. Giusti Minimal surfaces and functions of bounded variation, Monographs in Mathematics, Birkhäuser, 80 (1984).

[10] C. Liverani, On contact Anosov flows, Ann. of Math. 159 (2004) 1275-1312.

[11] G. Keller, Generalized bounded variation and applications to piecewise monotonic transformations, Z. Wahrscheinlichkeitstheorie verw. Geb. 69 (1985), 461-478.

[12] M. Rychlik, Bounded variation and invariant measures, Studia Math. 69 (1983), 69-80.

[13] R. Zweimüller, Ergodic structure and invariant densities of non-Markovian interval maps with indifferent fixed points, Nonlineariy 11 (1998), 1263-1267.

[14] R. Zweimüller, Ergodic properties of infinite measure-preserving interval maps with indifferent fixed points, Ergod. Th. \& Dynam. Sys. 20 (2000), 1519-1549. 